\title{
Deslocamentos Isométricos em Espaços de Banach
}

\author{
Fernando Dallapé Madeira
}

DisSERTAÇÃO APRESENTADA

$\mathrm{AO}$

Instituto De Matemática e Estatística

DA

Universidade DE SÃo PAUlo

PARA

OBTENÇÃO DO TÍTULO

$\mathrm{DE}$

Mestre em MatemáticA

Programa: Matemática

Orientador: Prof. Dr. Elói Medina Galego

Durante o desenvolvimento deste trabalho o autor recebeu auxílio financeiro da CAPES e do $\mathrm{CNPq}$

São Paulo, Agosto de 2014 



\section{Deslocamentos Isométricos em Espaços de Banach}

Esta versão da dissertação contém as correções e alterações sugeridas pela Comissão Julgadora durante a defesa da versão original do trabalho, realizada em 15/08/2014. Uma cópia da versão original está disponível no Instituto de Matemática e Estatística da Universidade de São Paulo.

Comissão Julgadora:

- Prof. Dr. Elói Medina Galego (orientador) - USP

- Prof. Dr. Antonio Roberto Silva - UFRJ

- Prof. Dr. Raymundo Luiz de Alencar - ITA 



\section{Agradecimentos}

Agradeço sobretudo à Deus, por me dar saúde e oportunidades para chegar nesse momento tão feliz da minha vida.

Agradeço ao meu orientador por ser sempre muito atencioso e por estar envolvido com esse trabalho até o fim. Graças à ele, estou muito mais maduro e autônomo para ampliar meu conhecimento.

Agradeço aos meus queridos pais, Sônia e Victor, por serem pessoas tão amáveis e carinhosas e não terem medido esforços para me apoiar nessa conquista.

Agradeço à minha "companheira de sempre", Angélica Turaça, por me dar muitas demonstrações de que a vida é bela e graciosa. Esse anjo me inspira todos os dias a mudar e ser cada vez melhor.

Agradeço ao meu irmão pelos momentos divertidos e alegres, esses permitiram um equilíbrio diante de toda seriedade que este trabalho demandou.

Agradeço aos meus amigos Anderson, Antonio, Artur, Renan Marcel, Renan Sanches, Romenig, Sheldon, Silmara e Thiago.

Agradeço aos professores Antonio Roberto Silva e Raymundo Luiz de Alencar por terem aceitado fazer parte da banca avaliadora e por suas observações, sugestões e correções.

Agradeço ao CNPq e à CAPES pelo apoio financeiro. 


\section{Resumo}

MADEIRA, F. D. Deslocamentos Isométricos em Espaços de Banach. 2014. 84 f. Dissertação (Mestrado) - Instituto de Matemática e Estatística, Universidade de São Paulo, São Paulo, 2014.

Neste trabalho, estudamos a existência de deslocamentos isométricos ${ }^{1}$ no produto de espaços de Banach, munido tanto com a norma $\|\cdot\|_{\infty}$ como com a norma $\|\cdot\|_{1}$. Em alguns casos, assumimos que pelo menos um dos espaços envolvidos no produto é estritamente convexo e isso deu origem a resultados interessantes. Ainda, exploramos os deslocamentos isométricos em $C(X)$, mostrando como classificá-los de acordo com dois tipos.

Palavras-chave: deslocamentos isométricos, deslocamentos isométricos no espaço produto, deslocamentos isométricos em $C(X)$, deslocamentos reversos, existência de deslocamentos em espaços de Banach.

\footnotetext{
${ }^{1}$ Os termos deslocamento isométrico e deslocamento reverso foram traduzidos do inglês, respectivamente, dos termos "isometric shift"e"backward shift".
} 


\section{Abstract}

MADEIRA, F. D. Deslocamentos Isométricos em Espaços de Banach. 2014. 84 f. Dissertação (Mestrado) - Instituto de Matemática e Estatística, Universidade de São Paulo, São Paulo, 2014.

In this work, we study the existence of isometric shifts on the product of Banach spaces, equipped with the norms $\|\cdot\|_{\infty}$ or $\|\cdot\|_{1}$. In some cases, we assume that at least one of these spaces on the product is strictly convex and obtain interesting results. Besides, we explore isometric shifts on $C(X)$, showing how to sort them on two groups.

Keywords: Isometric shifts, isometric shifts on the product of Banach spaces, isometric shifts on $C(X)$, backward shifts, existence of isometric shifts on Banach spaces. 


\section{Sumário}

Notação $\quad$ v

Introdução

1 Preliminares 1

1.1 Topologia - Conceitos e resultados básicos . . . . . . . . . . . . . . . 1

1.1.1 Axiomas de separação . . . . . . . . . . . . . . . . . . . 1

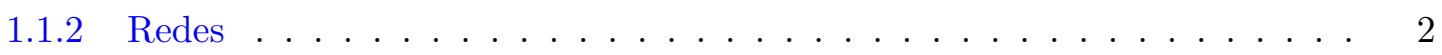

1.1.3 Compactificações de um espaço topológico . . . . . . . . . . . . . . . . . . . . 3

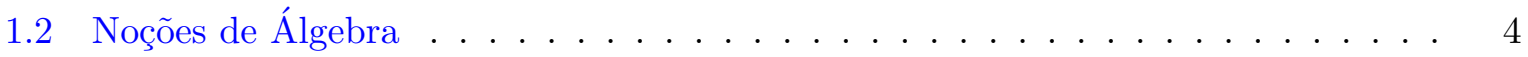

1.3 Noções de Análise Funcional . . . . . . . . . . . . . . . . . . . 8

2 Deslocamentos isométricos em $c \oplus_{\infty} X \quad 12$

2.1 Introdução . . . . . . . . . . . . . . . . . . . . . . . . . . . 12

2.2 Definição e algumas propriedades elementares dos deslocamentos isométricos . . 12

2.3 Construção de deslocamentos isométricos . . . . . . . . . . . . . . . 15

2.4 Deslocamentos isométricos em $c \oplus_{\infty} X \ldots \ldots \ldots \ldots \ldots \ldots \ldots$

3 Deslocamentos isométricos em $E \oplus_{1} F \quad 32$

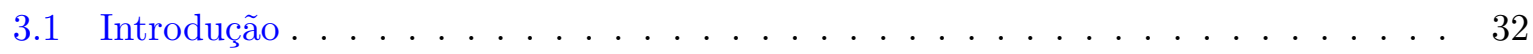

3.2 Definições e resultados básicos . . . . . . . . . . . . . . . . . 32

3.3 Espaços sem deslocamentos isométricos . . . . . . . . . . . . . . . . . 39

3.4 Deslocamentos reversos em Espaços de Banach . . . . . . . . . . . . . . . . . 43

4 Deslocamentos isométricos em $C(X) \quad 49$

4.1 Introdução . . . . . . . . . . . . . . . . . . . . . . . . 49

4.2 Isometrias em $C(X) \ldots \ldots \ldots \ldots \ldots \ldots \ldots \ldots \ldots$

4.3 Classificação dos deslocamentos isométricos em $C(X) \ldots \ldots \ldots \ldots$. . . . . . 55

4.4 Estudo dos deslocamentos isométricos tipo I . . . . . . . . . . . . . . . . 62

4.5 Estudo dos deslocamentos isométricos tipo II . . . . . . . . . . . . . . . 69

$\begin{array}{ll}\text { Referências Bibliográficas } & 73\end{array}$ 


\section{Notação}

$\begin{array}{ll}\mathbb{N} & \text { o conjunto dos números inteiros estritamente positivos } \\ \mathbb{K} & \text { os corpos } \mathbb{R} \text { ou } \mathbb{C} \\ X & \text { um espaço vetorial normado sobre } \mathbb{K} \text { (exceto no Capítulo 4) } \\ \ell_{\infty} & \text { o espaço de Banach das sequências em } \mathbb{K} \text { que são limitadas } \\ c & \text { o espaço de Banach das sequências em } \mathbb{K} \text { que são convergentes } \\ c_{0} & \text { o espaço de Banach das sequências em } \mathbb{K} \text { que convergem para } 0 \\ {\left[v_{1}, v_{2}, \ldots, v_{n}\right]} & \text { o subespaço gerado pelas combinações lineares dos vetores } v_{1}, v_{2}, \ldots, v_{n} \\ \mathbb{K} v & \text { o subespaço }[v] \\ B_{X} & \text { a bola unitária fechada de um espaço normado } X \\ S_{X} & \text { a esfera unitária de um espaço normado } X \\ X^{*} & \text { o espaço vetorial normado dos funcionais lineares e contínuos em } X \\ X & \text { o espaço quociente de } X \text { por um subespaço } M \\ X \oplus Y & \text { o espaço vetorial produto de dois espaços vetoriais normados } X \text { e } Y \\ X \oplus_{\infty} Y & \text { o espaço produto munido com a norma }\|\cdot\|_{\infty} \\ X \oplus_{1} Y & \text { o espaço produto munido com a norma }\|\cdot\|_{1} \\ \operatorname{Re}(z) & \text { a parte real de um número complexo } z \\ \operatorname{Im}(z) & \text { a parte imaginária de um número complexo } z \\ T & \text { uma transformação linear } \\ K e r T & \text { o núcleo de uma tranformação linear } T \\ \operatorname{Im} T & \text { a imagem de uma tranformação linear } T\end{array}$

Particularmente, para $p \in \mathbb{R}, 1 \leq p<\infty$ :

$\ell_{p} \quad$ o espaço de Banach das sequências $\left(x_{n}\right)_{n}$ em $\mathbb{K}$, tais que $\sum_{j=1}^{\infty}\left|x_{j}\right|^{p}<\infty$

\section{Exclusivamente no Capítulo 4:}

$X$

$C(X)$

$\beta X$ um espaço topológico compacto e Hausdorff

o espaço das funções contínuas em $X$ e com valores em $\mathbb{K}$

o compactificado de Stone-Cech de $X$ 


\section{Introdução}

Neste trabalho, estudaremos uma classe de operadores definidos em espaços de Banach, os deslocamentos isométricos. Esses operadores vem sendo explorados nas pesquisas e, em quase todos artigos que tratam do assunto, identificamos uma questão em comum. Essa questão diz respeito a existência de deslocamentos isométricos em determinados espaços de Banach.

O primeiro matemático a dar uma definição geral de deslocamentos em espaços de Banach foi R. Crownover, em um artigo publicado no ano de 1972. Essa definição não apenas abrangia isometrias, mas outros operadores contínuos e injetores. Esses deslocamentos são muitas vezes citados como deslocamentos de Crownover ou deslocamentos clássicos. Em 1988, outro importante matemático, Holub, publicou um artigo sobre deslocamentos. Nesse artigo, Holub reformulou e criou definições de deslocamentos, como por exemplo a noção de deslocamento reverso. Essa abordagem do assunto na década de 80 deu ênfase também para os deslocamentos isométricos, nosso objeto de estudo neste trabalho. Muitas questões e conjecturas foram feitas, principalmente, focadas na possibilidade de construção de deslocamentos isométricos em espaços de Banach dados.

Recentemente, no artigo da referência [5], Pei-Kee Lin explorou a existência de deslocamentos isométricos no produto de dois espaços de Banach, considerado com a norma $\|\cdot\|_{\infty}$. Nesse artigo, é nítido que quando os espaços envolvidos no produto são estritamente convexos, tiramos fortes conclusões a respeito dos deslocamentos isométricos e isso nos dá uma sensação de controle ou domínio sobre o estudo. A segunda parte do artigo é pautada no estudo de espaços da forma $c \oplus_{\infty} X$, sendo $X$ um espaço de Banach qualquer e $c$ o espaço das sequências convergentes. Ainda no artigo, o autor deixou o seguinte problema em aberto:

Questão 1. O espaço produto $c \oplus_{\infty} c_{0}$ admite um deslocamento isométrico quando considerado como $\mathbb{R}$-espaço vetorial?

No Capítulo 2, responderemos a questão 1 no caso complexo, tal como foi feito em [3]. Seguindo a referência [5], dedicaremos nossa atenção para os espaços $c \oplus_{\infty} X$, particularmente quando $X$ for estritamente convexo. Nesse caso, notaremos algumas propriedades e consequências da hipótese de $c \oplus_{\infty} X$ admitir ao menos um deslocamento isométrico. Ainda apresentaremos um teorema capaz de gerar exemplos de deslocamentos, na medida em que traz condições suficientes para $c \oplus_{\infty} X$ admitir um deslocamento isométrico.

No Capítulo 3, estudaremos o espaço produto munido com a norma $\|\cdot\|_{1}$. Devemos ter em mente que os deslocamentos isométricos são operadores que preservam a norma, portanto este caso demanda um tratamento particular. Nosso interesse, nesse capítulo, será estudar especificamente o produto de espaços estritamente convexos - estabelecendo, quando possível, 
comparações com os resultados do capítulo 2. Seguindo o que está feito em [3], mostraremos que um deslocamento isométrico definido no espaço produto é fatorável e sua imagem é um subespaço retangular. Além disso, mostraremos que, para $1<p \neq q<\infty$, os espaços $\ell_{p} \oplus_{\infty} \ell_{q}$ e $\ell_{p} \oplus_{1} \ell_{q}$ não admitem deslocamentos isométricos. Na última seção, definiremos os deslocamentos reversos e mostraremos como esses operadores estão relacionados aos deslocamentos isométricos. Para saber mais sobre os deslocamentos reversos, podemos consultar [7].

No Capítulo 4, estudaremos os deslocamentos isométricos no espaço $C(X)$, sendo $X$ um espaço topológico compacto e Hausdorff. A importância de explorar os deslocamentos em $C(X)$ está em buscar mais exemplos sobre esses operadores e, também, exemplos de espaços que não os admitem. Iniciaremos o capítulo 4 enunciando e demonstrando um importante teorema, o qual pode ser visto em [8]. Com a ajuda desse teorema, construiremos funções induzidas por uma isometria definida em $C(X)$, as quais possibilitam descrevê-la. Após apresentar esse teorema, trataremos de como classificar um deslocamento isométrico definido em $C(X)$ segundo dois tipos. Feito isso, partiremos para o estudo de cada um dos tipos, dando diversos exemplos.

Antes de tudo, o Capítulo 1 tem o objetivo de trazer alguns conhecimentos que serão fundamentais para compreensão deste trabalho. 


\section{Capítulo 1}

\section{Preliminares}

\subsection{Topologia - Conceitos e resultados básicos}

As noções de topologia presentes nesta seção serão fundamentais para entender o capítulo 4. Inicialmente, apresentaremos alguns axiomas de separação e enunciaremos o Lema de Urysohn. Depois disso, definiremos o que é uma rede e veremos alguns resultados básicos. Por fim, trataremos das compactificações de Alexandroff e Stone-Cech.

\subsubsection{Axiomas de separação}

Definição 1. (Axiomas de Separação)([2], pg.36)

Seja $(X, \tau)$ um espaço topológico. Dizemos que:

S1) $(X, \tau)$ é $\mathbb{T}_{1}$ se para cada $x, y \in X$ com $x \neq y$ existem $U_{x}, U_{y} \in \tau$ tais que $U_{x} \cap\{x, y\}=\{x\}$ e $U_{y} \cap\{x, y\}=\{y\}$

S2) $(X, \tau)$ é $\mathbb{T}_{2}$, ou Hausdorff, se para cada $x, y \in X$ com $x \neq y$ existem $U_{x}, U_{y} \in \tau$ tais que $x \in U_{x}, y \in U_{y}$ e $U_{x} \cap U_{y}=\emptyset ;$

S3) $(X, \tau)$ é $\mathbb{T}_{3}$ se para cada $F \subseteq X$ fechado e para todo $x \in X$ tal que $x \notin F$ existem $U, V \in \tau$ tais que $F \subseteq U, x \in V$ e $U \cap V=\emptyset$;

S4) $(X, \tau)$ é $\mathbb{T}_{3 \frac{1}{2}}$ se para cada $F \subseteq X$ fechado e para todo $x \in X$ tal que $x \notin F$ existe uma função contínua $f: X \longrightarrow[0,1]$ satisfazendo $f(x)=0$ e $f(t)=1$, para todo $t \in F$;

S5) $(X, \tau)$ é $\mathbb{T}_{4}$ se para cada par de conjuntos fechados e disjuntos $A, B \subseteq X$ existem $U, V \in \tau$ tais que $A \subseteq U, B \subseteq V$ e $U \cap V=\emptyset$;

S6) $(X, \tau)$ é um espaço de Tychonoff se $(X, \tau)$ é $\mathbb{T}_{2}$ e $\mathbb{T}_{3 \frac{1}{2}}$.

S7) $(X, \tau)$ é normal se $(X, \tau)$ é $\mathbb{T}_{1}$ e $\mathbb{T}_{4}$.

Teorema 1. ([2], pg.125) Todo espaço topológico compacto e Hausdorff é normal.

No Capítulo 4, o Lema de Urysohn será usado várias vezes, principalmente na demonstração por absurdo. Logo é muito importante conhecer esse enunciado com clareza. 
Teorema 2. (Lema de Urysohn)([2], pg.41) Seja X um espaço topológico normal, isto é, um espaço topológico $\mathbb{T}_{1} e \mathbb{T}_{4}$. Para cada par de conjuntos fechados e disjuntos $A$ e $B$, existe uma função contínua $f: X \longrightarrow[0,1]$ de maneira que $f(x)=0$, para todo $x \in A$ e $f(y)=1$, para todo $y \in B$.

\subsubsection{Redes}

Definição 2. Um conjunto não vazio $D$ é dirigido se existir uma relação $\preceq$ em $D$, satisfazendo

(1) $\alpha \preceq \alpha$, para todo $\alpha \in D$;

(2) Para todos $\alpha, \beta, \gamma \in D$, se $\alpha \preceq \beta$ e $\beta \preceq \gamma$, então $\alpha \preceq \gamma$;

(3) Para cada par $\alpha, \beta$ de elementos em $D$, existe $\gamma_{\alpha, \beta}$ em $D$ tal que $\alpha \preceq \gamma_{\alpha, \beta}$ e $\beta \preceq \gamma_{\alpha, \beta}$.

Definição 3. Sejam $X$ um conjunto e $D$ um conjunto dirigido. Uma rede em $X$ é uma função definida da seguinte forma:

$$
f: D \longrightarrow X
$$

$O$ conjunto $D$ é chamado de conjunto índice para a rede $f$.

Observação 1. Para $\alpha \in D$, dizemos que $f(\alpha)$ é o $\alpha$-ésimo termo de $f$. Analogamente à forma como denotamos as sequências, é usual representar o $\alpha$-ésimo termo de $f$ por $x_{\alpha}$. A rede $f$ também pode ser denotada por $\left(x_{\alpha}\right)_{\alpha \in D}$.

Definição 4. Seja $\left(x_{\alpha}\right)_{\alpha \in D}$ uma rede em um espaço topológico $X$ e seja $x$ um elemento de $X$. Dizemos que $\left(x_{\alpha}\right)_{\alpha \in D}$ converge para $x$ se, para todo aberto $U$ contendo $x$, existe $\alpha_{U} \in D$ tal que $x_{\alpha} \in U$ para qualquer $\alpha \in D$, tal que $\alpha_{U} \preceq \alpha$. Nesse caso $x$ é chamado de limite de $\left(x_{\alpha}\right)_{\alpha \in D} e$ usamos a notação

$$
x_{\alpha} \rightarrow x \text { ou } \lim _{\alpha} x_{\alpha}=x .
$$

Proposição 1. ([1], pg.145) Um espaço topológico é Hausdorff se, e somente se, vale a unicidade do limite para cada rede convergente em $X$.

Proposição 2. ([1], pg.146) Seja $S$ um subconjunto de um espaço topológico $X$ e seja $x$ um elemento de $X$. Então $x \in \bar{S}$ se, e somente se, existe uma rede de elementos de $S$ convergindo para $x$.

Proposição 3. ([1],pg.146) Um subconjunto $S$ de um espaço topológico $X$ é fechado se,e somente se, $S$ contém os limites de todas redes convergentes, cujos elementos elementos estão em $S$.

Proposição 4. ([1],pg.146) Sejam $X$ e $Y$ espaços topológicos e seja $f: X \longrightarrow Y$ uma função de $X$ em $Y$.

(a) A função $f$ é contínua em $x_{0} \in X$ se, e somente se, $f\left(x_{\alpha}\right) \rightarrow f\left(x_{0}\right)$ para toda rede $\left(x_{\alpha}\right)_{\alpha \in D}$ convergindo para $x_{0}$;

(b) A função $f$ é contínua em $X$ se, e somente se, para todo $x \in X$ e para toda rede $\left(x_{\alpha}\right)_{\alpha \in D}$ convergindo para $x$, temos $f\left(x_{\alpha}\right) \rightarrow f(x)$. 
Definição 5. Um subconjunto $J$ de um conjunto dirigido $I$ é cofinal em $I$ se para cada $\alpha \in I$, existe $\beta_{\alpha} \in J$ de modo que $\alpha \preceq \beta_{\alpha}$.

Definição 6. Suponhamos que $X$ seja um conjunto, I seja um conjunto dirigido e suponhamos que $f: I \longrightarrow X$ seja uma rede. Além disso, suponhamos que $J$ seja um conjunto dirigido e $g: J \longrightarrow I$ seja uma função de maneira que

(1) Para todos $\beta_{1}, \beta_{2} \in J$, se $\beta_{1} \preceq \beta_{2}$, então $g\left(\beta_{1}\right) \preceq g\left(\beta_{2}\right)$ em I;

(2) $g(J)$ é cofinal em $I$.

Então a rede $f \circ g: J \longrightarrow X$ é chamada subrede de $f$.

Proposição 5. ([1],pg.150) Seja $D$ um conjunto dirigido $e\left(x_{\alpha}\right)_{\alpha \in D}$ uma rede em um conjunto $X$.

(a) A rede $\left(x_{\alpha}\right)_{\alpha \in D}$ é subrede de si mesma;

(b) Toda subrede de $\left(x_{\alpha}\right)_{\alpha \in D}$ é uma rede em $X$;

(c) Toda subrede de uma subrede de $\left(x_{\alpha}\right)_{\alpha \in D}$ é também uma subrede de $\left(x_{\alpha}\right)_{\alpha \in D}$;

(d) Se $X$ é um espaço topológico e $\left(x_{\alpha}\right)_{\alpha \in D}$ converge para um elemento $x \in X$, então toda subrede de $\left(x_{\alpha}\right)_{\alpha \in D}$ converge para $x$;

(e) Se $X$ é um espaço topológico e existe um elemento $x \in X$ tal que toda subrede de $\left(x_{\alpha}\right)_{\alpha \in D}$ tem uma subrede convergindo para $x$, então $x_{\alpha} \rightarrow x$.

Proposição 6. ([1],pg.152) Um subconjunto $S$ de um espaço topológico $X$ é compacto se, e somente se, toda rede em $S$ admite uma subrede convergente com limite em $S$.

Essas noções sobre redes são mais do que suficientes para entender este trabalho, mas podemos encontrar exemplos e mais resultados interessantes em [1].

\subsubsection{Compactificações de um espaço topológico}

Nesta subseção, veremos dois casos especiais de compactificação. Sejam $(X, \tau)$ um espaço topológico não compacto e $\infty=\{X\}$. Logo $\infty$ não é um elemento do conjunto $X$. Vamos considerar

$$
\tau^{\prime}=\tau \cup\{A \cup\{\infty\}: A \in \mathcal{P}(X) \text { e } X \backslash A \text { é fechado e compacto em } X\} \text {. }
$$

Definimos $X^{*}=X \cup\{\infty\}$, é simples verificar que $\left(X^{*}, \tau^{\prime}\right)$ é um espaço topológico e que $X$ é denso em $X^{*}$. Com essa notação, enunciaremos o próximo teorema e a próxima definição.

Teorema 3. (Alexandroff) ([11], pg.150) O espaço topológico $X^{*}$ é compacto e tem $X$ como subespaço topológico. Além disso, $X^{*}$ é Hausdorff se, e somente se, $X$ é localmente compacto e Hausdorff.

Definição 7. O espaço topológico $X^{*}$ é chamado de compactificado de Alexandroff de $X$, ou ainda, compactificação de um ponto do espaço $X$. 
Ainda na referência [11], nas páginas 151, 152 e 153, prova-se a existência do compactificado de Stone-Cech. A seguir, vamos defini-lo.

Definição 8. Seja $X$ um espaço de Tychonoff, isto é, um espaço topológico $\mathbb{T}_{2}$ e $\mathbb{T}_{3 \frac{1}{2}}$. Seja $Y$ um espaço topológico compacto, Hausdorff e que tenha $X$ como subespaço topológico denso. Dizemos que $Y$ é um compactificado de Stone-Cech para $X$ se, para todo $K$ compacto, Hausdorff e para toda função contínua $f: X \longrightarrow K$, existe $g: Y \longrightarrow K$ contínua e que estende $f$.

\subsection{Noções de Álgebra}

Nesta seção, vamos trabalhar com a noção de independência sobre os racionais e obter algumas propriedades algébricas que serão fundamentais para uma construção no segundo capítulo. Essas propriedades encontram-se em [3], na seção de preliminares. Antes de iniciarmos, vamos definir alguns conceitos básicos.

Definição 9. Seja $G=\left\{y_{1}, y_{2}, \ldots, y_{n}\right\}$ um conjunto finito de números reais. Dizemos que $G$ é independente sobre os racionais se, para todos inteiros $k_{1}, k_{2}, \ldots, k_{n}, k_{n+1}$ que satisfizerem

$$
k_{1} y_{1}+k_{2} y_{2}+\ldots+k_{n} y_{n}=k_{n+1}
$$

tivermos $k_{1}=k_{2}=\ldots=k_{n}=k_{n+1}=0$.

Essa definição trata apenas de subconjuntos finitos de $\mathbb{R}$, daí vem a necessidade de enunciar outra definição.

Definição 10. Seja $H$ um conjunto qualquer de números reais. Dizemos que $H$ é independente sobre os racionais se cada subconjunto finito de $H$ é independente sobre os racionais.

Em [4], página 257 - exemplo 65, demonstra-se o seguinte resultado:

Proposição 7. Seja $\left\{y_{1}, y_{2}, \ldots, y_{n}\right\}$ um conjunto finito de números reais, que seja independente sobre os racionais. Sejam $x_{1}, x_{2}, \ldots, x_{n}$ números reais e $\varepsilon>0$ dado. Então existem inteiros $k, m_{1}, m_{2}, \ldots, m_{n}$ tais que

$$
\left|x_{i}-k y_{i}-m_{i}\right|<\varepsilon, \text { para todo } i=1,2, \ldots, n \text {. }
$$

Através da proposição 7, vamos demonstrar a próxima proposição.

Proposição 8. Sejam $z_{1}, z_{2}, \ldots, z_{n}$ números complexos com valor absoluto 1. Vamos supor que $z_{t}=e^{2 \pi i y_{t}}$, para cada $t \in\{1,2, \ldots, n\}$. Além disso, suponhamos que o conjunto $\left\{y_{1}, y_{2}, \ldots, y_{n}\right\}$ seja independente sobre os racionais. Assim, dados $u_{1}, u_{2}, \ldots, u_{n}$ números complexos com valor absoluto 1 e $\varepsilon>0$, existe um inteiro $k$ tal que

$$
\left|u_{t}-z_{t}^{k}\right|<\varepsilon, \text { para todo } t=1,2, \ldots, n \text {. }
$$


Demonstração. Consideremos $S=\{z \in \mathbb{C}:|z|=1\}$. Sabemos que a função

$$
\begin{aligned}
f: \mathbb{R} & \longrightarrow S \\
x & \longmapsto e^{2 \pi i x}
\end{aligned}
$$

é contínua e sobrejetora. Tomemos $\varepsilon>0$ e $u_{1}, u_{2}, \ldots, u_{n} \in S$ de maneira arbitrária. Existem $x_{1}, x_{2}, \ldots, x_{n} \in \mathbb{R}$ tais que $u_{t}=f\left(x_{t}\right)$, para cada $t=1,2, \ldots, n$. Pela continuidade de $f$ nos pontos $x_{1}, x_{2}, \ldots, x_{n}$, existe $\delta>0$ tal que, para todo $x \in \mathbb{R}$,

$$
\text { se }\left|x-x_{t}\right|<\delta \text {, então }\left|f(x)-f\left(x_{t}\right)\right|<\varepsilon \text {, para cada } t \in\{1,2, \ldots, n\} \text {. }
$$

Pela proposição 7 , existem inteiros $k, m_{1}, m_{2}, \ldots, m_{n}$ tais que

$$
\left|x_{t}-k y_{t}-m_{t}\right|<\delta \text {, para todo } t=1,2, \ldots, n \text {. }
$$

Para cada $t \in\{1,2, \ldots, n\}$, vale:

$$
\begin{aligned}
\left|f\left(x_{t}\right)-f\left(k y_{t}+m_{t}\right)\right| & =\left|e^{2 \pi i x_{t}}-e^{2 \pi i k y_{t}} e^{2 \pi i m_{t}}\right|= \\
& =\left|u_{t}-\left(e^{2 \pi i y_{t}}\right)^{k}\right|= \\
& =\left|u_{t}-z_{t}^{k}\right|
\end{aligned}
$$

Portanto, para cada $t \in\{1,2, \ldots, n\}$, temos

$$
\left|u_{t}-z_{t}^{k}\right|<\varepsilon
$$

e isso conclui a demonstração.

Seja $z$ um número complexo de módulo 1. Vamos escrevê-lo na forma $z=e^{2 \pi i y}$, com $0 \leq y<1$, daí dizemos que $y$ é o argumento de $z$ e denotamos $y=\arg (z)$. Vamos continuar a usar a notação da proposição 8 e utilizar $S$ para denotar o conjunto dos números complexos de módulo 1. Para $n \in \mathbb{Z}$ positivo, consideremos

$$
S^{n}=\left\{\left(z_{1}, z_{2}, \ldots, z_{n}\right): z_{t} \in S, \text { para todo } t=1,2, \ldots, n\right\}
$$

Consideremos ainda, a função $d_{\infty}: S^{n} \times S^{n} \longrightarrow \mathbb{R}_{+}$, dada por:

$$
d_{\infty}\left(\left(z_{1}, z_{2}, \ldots, z_{n}\right),\left(w_{1}, w_{2}, \ldots, w_{n}\right)\right)=\sup \left\{\left|z_{t}-w_{t}\right|: t=1,2, \ldots, n\right\}
$$

Essa função define uma métrica e uma topologia para $S^{n}$. Tendo isso em mente e a proposição 8, é imediato verificar o próximo resultado.

Proposição 9. Sejam $n$ um inteiro positivo e $z_{1}, z_{2}, \ldots, z_{n}$ números complexos de módulo 1. Suponhamos que o conjunto $\left\{\arg \left(z_{1}\right), \arg \left(z_{2}\right), \ldots, \arg \left(z_{n}\right)\right\}$ seja independente sobre os racionais. Então o conjunto

$$
H=\left\{\left(z_{1}^{k}, z_{2}^{k}, \ldots, z_{n}^{k}\right): k \in \mathbb{Z}\right\}
$$

é denso em $S^{n}$. 
O próximo resultado decorre das proposições 7 e 9.

Proposição 10. Sejam $n$ um inteiro positivo e $z_{1}, z_{2}, \ldots, z_{n}$ números complexos de módulo 1. Suponhamos que o conjunto $\left\{\arg \left(z_{1}\right), \arg \left(z_{2}\right), \ldots, \arg \left(z_{n}\right)\right\}$ seja independente sobre os racionais. Então o conjunto

$$
H=\left\{\left(z_{1}^{k}, z_{2}^{k}, \ldots, z_{n}^{k}\right): k=1,2, \ldots\right\}
$$

é denso em $S^{n}$. Assim, dados $\left(x_{1}, x_{2}, \ldots, x_{n}\right) \in S^{n}, \varepsilon>0$ e um inteiro $k_{0}$, existe um inteiro $k>k_{0}$ de modo que

$$
\left|x_{t}-z_{t}^{k}\right|<\varepsilon, \text { para todo } t=1,2, \ldots, n \text {. }
$$

Demonstração. Vamos assumir $\arg \left(z_{t}\right)=y_{t}$, para cada $t=1,2, \ldots, n$. Para demonstrarmos que $H$ é denso em $S^{n}$, basta provarmos que $H$ é denso em $\left\{\left(z_{1}^{k}, z_{2}^{k}, \ldots, z_{n}^{k}\right): k \in \mathbb{Z}\right\}$ e usar a proposição 9. Então, consideremos $\left(z_{1}^{l}, z_{2}^{l}, \ldots, z_{n}^{l}\right)$, com $l<0$ e $\varepsilon>0$ dados. Pela continuidade da função exponencial, existe $\delta>0$ de maneira que,

$$
\text { para todo } x \in \mathbb{R} \text {, se }|x|<\delta \text {, então }\left|e^{2 \pi i x}-1\right|<\varepsilon \text {. }
$$

Pela proposição 7 , existem $k, m_{1}, m_{2}, m \ldots, m_{t}$ inteiros tais que

$$
\left|\frac{\delta}{4|l|}-k y_{t}-m_{t}\right|<\frac{\delta}{8|l|}, \text { para todo } t=1,2, \ldots, n
$$

E obviamente $k \neq 0$ na relação acima. Assim, para cada $t \in\{1,2, \ldots, n\}$, valem

$$
\left\{\begin{array}{c}
-\frac{\delta}{8}-\frac{\delta}{4}<-k|l| y_{t}-m_{t}|l|<\frac{\delta}{8}-\frac{\delta}{4} \\
\frac{\delta}{4}-\frac{\delta}{8}<k|l| y_{t}+m_{t}|l|<\frac{\delta}{4}+\frac{\delta}{8}
\end{array}\right.
$$

Dessa forma, por (1.1) segue que

$$
\left|e^{2 \pi i(-k)|l| y_{t}}-1\right|<\varepsilon \mathrm{e}\left|e^{2 \pi i k|l| y_{t}}-1\right|<\varepsilon, \text { para todo } t=1,2, \ldots, n .
$$

Portanto, temos

$$
\left|z_{t}^{-k|l|}-1\right|<\varepsilon \text { e }\left|z_{t}^{k|l|}-1\right|<\varepsilon \text {, para todo } t=1,2, \ldots, n \text {. }
$$

Como $\left|z_{t}^{l}\right|=1$, para todo $t \in\{1,2, \ldots, n\}$, concluímos que

$$
\left|z_{t}^{-k|l|+l}-z_{t}^{l}\right|<\varepsilon \text { e }\left|z_{t}^{k|l|+l}-z_{t}^{l}\right|<\varepsilon \text {, para todo } t \in\{1,2, \ldots, n\} .
$$

Isso significa que

$$
d_{\infty}\left(\left(z_{1}^{-k|l|+l}, z_{2}^{-k|l|+l}, \ldots, z_{n}^{-k|l|+l}\right),\left(z_{1}^{l}, z_{2}^{l}, \ldots, z_{n}^{l}\right)\right)<\varepsilon
$$

e

$$
d_{\infty}\left(\left(z_{1}^{k|l|+l}, z_{2}^{k|l|+l}, \ldots, z_{n}^{k|l|+l}\right),\left(z_{1}^{l}, z_{2}^{l}, \ldots, z_{n}^{l}\right)\right)<\varepsilon .
$$


Evidentemente $\left(z_{1}^{-k|l|+l}, z_{2}^{-k|l|+l}, \ldots, z_{n}^{-k|l|+l}\right) \in H$ ou $\left(z_{1}^{k|l|+l}, z_{2}^{k|l|+l}, \ldots, z_{n}^{k|l|+l}\right) \in H$, assim o caso $l<0$ está feito. Notemos que se $\left(z_{1}^{l}, z_{2}^{l}, \ldots, z_{n}^{l}\right) \in S^{n}$ e $l>0$, então $\left(z_{1}^{l}, z_{2}^{l}, \ldots, z_{n}^{l}\right) \in H$ e não há nada para fazer. $\mathrm{O}$ caso $l=0$ não precisa ser feito, pois o ponto $\left(z_{1}^{0}, z_{2}^{0}, \ldots, z_{n}^{0}\right)$ não é isolado em $S^{n}$. Assim, concluímos que $H$ é denso em $S^{n}$. O restante do enunciado segue imediatamente do fato de $H$ ser denso em $S^{n}$, pois dado $\left(x_{1}, x_{2}, \ldots, x_{n}\right) \in S^{n}$, cada vizinhança desse ponto contém uma infinidade de pontos de $H$.

Proposição 11. Sejam $n>0$, um inteiro positivo $e\left\{y_{1}, y_{2}, \ldots, y_{n}\right\}$ um conjunto de números reais independente sobre os racionais. Vamos ainda definir, $z_{t}=e^{2 \pi i y_{t}}$ para cada $t \in\{1,2, \ldots, n\}$. Daí, dados $x_{1}, x_{2}, \ldots, x_{n} \in \mathbb{C} \backslash\{0\}, \varepsilon>0$ e um inteiro $k_{0}$, existe $k \in \mathbb{N}, k>k_{0}$ tal que

$$
|| x_{t}\left|-x_{t} z_{t}^{-k}\right|<\varepsilon, \text { para todo } t=1,2, \ldots, n \text {. }
$$

Demonstração. Consideremos $M=\sup \left\{\left|x_{1}\right|,\left|x_{2}\right|, \ldots,\left|x_{n}\right|\right\}>0$. Pela proposição 10, existe um natural $k>k_{0}$ de maneira que

$$
\left|\frac{x_{t}}{\left|x_{t}\right|}-z_{t}^{k}\right|<\frac{\varepsilon}{M} \text {, para todo } t=1,2, \ldots, n \text {. }
$$

Portanto, para cada $t \in\{1,2, \ldots, n\}$, temos

$$
\begin{aligned}
|| x_{t}\left|-x_{t} z_{t}^{-k}\right| & =\left|\frac{\left|x_{t}\right| z_{t}^{k}}{z_{t}^{k}}-\frac{x_{t}}{z_{t}^{k}}\right|=\frac{|| x_{t}\left|z_{t}^{k}-x_{t}\right|}{\left|z_{t}^{k}\right|}= \\
& =\frac{|| x_{t}\left|z_{t}^{k}-x_{t}\right|}{1}=\left|x_{t}\right|\left|z_{t}^{k}-\frac{x_{t}}{\left|x_{t}\right|}\right|< \\
& <\left|x_{t}\right| \frac{\varepsilon}{M} \leq \varepsilon .
\end{aligned}
$$

Logo, $k$ é o natural procurado.

Notemos que, na proposição 11 , se para algum $t \in\{1,2, \ldots, n\}$ tivéssemos $x_{t}=0$, a tese seria válida da mesma forma. De fato, se $x_{t}=0$, qualquer $k \in \mathbb{N}$ satisfaz

$$
|| x_{t}\left|-x_{t} z_{t}^{-k}\right|<\varepsilon
$$

Por isso, decorre da proposição 11 o seguinte corolário:

Corolário 1. Sejam $n>0$, um inteiro positivo e $\left\{y_{1}, y_{2}, \ldots, y_{n}\right\}$ um conjunto de números reais independente sobre os racionais. Com isso, vamos definir $z_{t}=e^{2 \pi i y_{t}}$ para cada $t \in\{1,2, \ldots, n\}$. Daí, dados $x_{1}, x_{2}, \ldots, x_{n} \in \mathbb{C}, \varepsilon>0$ e um inteiro $k_{0}$, existe $k \in \mathbb{N}, k>k_{0}$ tal que

$$
|| x_{t}\left|-x_{t} z_{t}^{-k}\right|<\varepsilon \text {, para todo } t=1,2, \ldots, n \text {. }
$$

O corolário 1 é necessário para compreensão do teorema 11. Além disso, é importante ter me mente a seguinte ideia. 
Observação 1. No capitulo 2, admitiremos a existência de um conjunto infinito (enumerável) de números reais que seja independente sobre os racionais. Notemos que a existência desse conjunto é garantida pelo fato de $\mathbb{R}$ ser um $\mathbb{Q}$-espaço vetorial de dimensão infinita. Essa última afirmação é uma simples consequência do conjunto dos reais ser não-enumerável.

\subsection{Noções de Análise Funcional}

Nesta seção, vamos tratar de alguns conceitos da Análise Funcional que serão importantes para entendermos este trabalho. Todos espaços vetoriais serão considerados sobre um corpo $\mathbb{K}$, sendo $\mathbb{K}=\mathbb{R}$ ou $\mathbb{K}=\mathbb{C}$. Nos capítulos 2 e 3 , a noção de espaço normado estritamente convexo será usada diversas vezes. Então é muito conveniente tratarmos desse conceito com bastante cuidado.

Definição 11. Seja $(X,\|\|$.$) um espaço vetorial normado. Dizemos que (X,\|\|$.$) é estritamente$ convexo se para todos $x, y \in X$ tais que $x \neq y$ e $\|x\|=\|y\|=1$, tivermos

$$
\left\|\frac{x+y}{2}\right\|<1
$$

Existem outras alternativas para definir espaços normados estritamente convexos. Por exemplo, a proposição a seguir revela uma condição equivalente a definição.

Proposição 12. Seja $(X,\|\|$.$) um espaço normado estritamente convexo. Sejam x, y \in X$ pontos distintos tais que $\|x\|=\|y\|=1$. Então para todo $\lambda \in \mathbb{R} \cap] 0,1[$ temos

$$
\|\lambda x+(1-\lambda) y\|<1
$$

Demonstração. Notemos que para $\lambda=\frac{1}{2}$, o resultado é exatamente o que diz a definição de espaço normado estritamente convexo e não há nada a fazer. Diante disso, precisamos estudar apenas os dois casos que seguem:

Primeiro Caso. Suponhamos que $\lambda \in] 0 ; \frac{1}{2}[$. Nesse caso notemos que

$$
\begin{aligned}
\|\lambda x+(1-\lambda) y\| & =\|\lambda x+y-\lambda y\|=\|\lambda x+\lambda y+y-2 \lambda y\|= \\
& =\left\|2 \lambda\left(\frac{x+y}{2}\right)+(1-2 \lambda) y\right\| \leq \\
& \leq\left\|2 \lambda\left(\frac{x+y}{2}\right)\right\|+\|(1-2 \lambda) y\|< \\
& <|2 \lambda|+|1-2 \lambda|=1 .
\end{aligned}
$$

Na última desigualdade usamos que $X$ é estritamente convexo. 
Segundo Caso. Suponhamos que $\lambda \in] \frac{1}{2} ; 1[$. Nesse caso notemos que

$$
\begin{aligned}
\|\lambda x+(1-\lambda) y\| & =\|\lambda x+y-\lambda y\|=\|x+y-\lambda x-\lambda y-x+2 \lambda x\|= \\
& =\left\|2(1-\lambda)\left(\frac{x+y}{2}\right)+(2 \lambda-1) x\right\| \leq \\
& \leq\left\|2(1-\lambda)\left(\frac{x+y}{2}\right)\right\|+\|(2 \lambda-1) x\|< \\
& <2|(1-\lambda)|+|(2 \lambda-1)|=1 .
\end{aligned}
$$

Novamente, na última desigualdade usamos que $X$ é estritamente convexo. Com todos os casos estudados, provamos a tese.

Usando somente a definição de espaços estritamente convexos demonstraremos as duas próximas proposições.

Proposição 13. Seja $(X,\|\|$.$) um espaço vetorial normado estritamente convexo sobre um corpo$ $\mathbb{K}$. Então, para todos $x, y \in X$ tais que $\|x+y\|=\|x\|+\|y\|$, existe $\alpha \geq 0$ tal que $x=\alpha y$ ou $y=\alpha x$.

Demonstração. Sejam $x$ e $y$ satisfazendo $\|x+y\|=\|x\|+\|y\|$. Se $x=0$ ou $y=0$, basta tomar $\alpha=0$ e acabou. Suponhamos $x \neq 0$ e $y \neq 0$, sem perda de generalidade suponhamos ainda que $0<\|y\| \leq\|x\|$. Consideremos $\alpha=\frac{\|x\|}{\|y\|} \geq 1$, assim:

$$
\begin{gathered}
\alpha\|x\|+\alpha\|y\|=\alpha\|x+y\|=\|\alpha x+\alpha y\|=\|\alpha x+\alpha y+x-x\|=\|x+\alpha y+(\alpha-1) x\| \leq \\
\|x+\alpha y\|+(\alpha-1)\|x\| \leq\|x\|+\alpha\|y\|+(\alpha-1)\|x\|=\alpha\|x\|+\alpha\|y\| .
\end{gathered}
$$

Donde concluímos que $\|x+\alpha y\|+(\alpha-1)\|x\|=\alpha\|x\|+\alpha\|y\|$, e assim $\|x+\alpha y\|=\|x\|+\alpha\|y\|$. Agora vamos dividir cada um dos lados da última equação por $\|x\|$ :

$$
\left\|\frac{x}{\|x\|}+\frac{\alpha y}{\|x\|}\right\|=1+\frac{\alpha\|y\|}{\|x\|}
$$

Tínhamos $\alpha=\frac{\|x\|}{\|y\|}$, e daí segue que

$$
\left\|\frac{x}{\|x\|}+\frac{y}{\|y\|}\right\|=2
$$

isso é o mesmo que

$$
\left\|\frac{\frac{x}{\|x\|}+\frac{y}{\|y\|}}{2}\right\|=1 .
$$

Por fim usamos que $X$ é estritamente convexo e concluímos da última iguladade que $\frac{x}{\|x\|}=\frac{y}{\|y\|}$, donde segue a tese. 
Proposição 14. Seja $(X,\|\|$.$) um espaço normado estritamente convexo sobre um corpo \mathbb{K}$. Para todos $x, y \in X$ distintos e não nulos, temos

$$
\left\|\frac{x+y}{2}\right\|<\max \{\|x\|,\|y\|\} \text {. }
$$

Demonstração. Se $\|x\|=\|y\|=c$, temos

$$
\left\|\frac{x}{c}\right\|=\left\|\frac{y}{c}\right\|=1
$$

Pelo fato de $X$ ser estritamente convexo, concluímos que

$$
\left\|\frac{x+y}{2 c}\right\|<1
$$

então

$$
\left\|\frac{x+y}{2}\right\|<c=\max \{\|x\|,\|y\|\} .
$$

Resta-nos, então, apenas o caso em que $\|x\| \neq\|y\|$, sem perda de generalidade suponhamos $c=\|x\|>\|y\|$. Nesse caso:

$$
\left\|\frac{x+y}{2 c}\right\| \leq\left\|\frac{x}{2 c}\right\|+\left\|\frac{y}{2 c}\right\|<1
$$

Com isso,

$$
\left\|\frac{x+y}{2}\right\|<c=\max \{\|x\|,\|y\|\} .
$$

Ao estudarmos todos os possíveis casos, temos a tese.

Corolário 2. Seja $(X,\|\|$.$) um espaço normado estritamente convexo sobre um corpo \mathbb{K}$. Não existem $x, y \in X$ não nulos tais que

$$
\|x\| \geq\|x+\alpha y\|, \forall \alpha \in \mathbb{K},|\alpha|=1
$$

Demonstração. Suponhamos, por absurdo, que existem $x, y \in X$ nas condições do enunciado e satisfazendo a desigualdade acima. Então:

$$
\|x\| \geq\|x-y\| \text { e }\|x\| \geq\|x+y\|
$$

Por essas desigualdades fica evidente que $x+y \neq 0$ e $x-y \neq 0$. Além disso, por valer $y \neq 0$, segue que $x+y \neq x-y$. Notemos que

$$
\|x\|=\left\|\frac{(x+y)+(x-y)}{2}\right\| \stackrel{\text { prop. } 14}{<} \max \{\|x+y\|,\|x-y\|\} \leq\|x\| .
$$

Uma contradição. Provamos a tese pela redução ao absurdo.

As demonstrações dos dois teoremas a seguir se encontram em [12], esses resultados nos darão suporte para demonstrar um importante resultado sobre os espaços $\ell_{p}$.

Teorema 4. Para quaisquer $x, y \in \ell_{p}$ com $2 \leq p<\infty$ e $q=\frac{p}{p-1}$, temos 
(a) $2\left(\|x\|^{p}+\|y\|^{p}\right) \leq\|x+y\|^{p}+\|x-y\|^{p} \leq 2^{p-1}\left(\|x\|^{p}+\|y\|^{p}\right)$;

(b) $2\left(\|x\|^{p}+\|y\|^{p}\right)^{q-1} \leq\|x+y\|^{q}+\|x-y\|^{q}$.

Teorema 5. Para quaisquer $x, y \in \ell_{p}$ com $1<p \leq 2$ e $q=\frac{p}{p-1}$, temos

(c) $2^{p-1}\left(\|x\|^{p}+\|y\|^{p}\right) \leq\|x+y\|^{p}+\|x-y\|^{p} \leq 2\left(\|x\|^{p}+\|y\|^{p}\right) ;$

(d) $\|x+y\|^{q}+\|x-y\|^{q} \leq 2\left(\|x\|^{p}+\|y\|^{p}\right)^{q-1}$.

Utilizando os teoremas 4 e 5 e a própria definição para espaços estritamente convexos, vamos demonstrar o próximo teorema.

Teorema 6. O espaço $\ell_{p}, 1<p<\infty$, com a norma usual é estritamente convexo.

Demonstração. Suponhamos $x, y \in \ell_{p}$, satisfazendo

$$
\|x\|=\|y\|=\left\|\frac{x+y}{2}\right\|=1 .
$$

Vamos analisar dois casos separadamente:

Primeiro Caso: $p \geq 2$. Pelo teorema 4 (a), temos:

$$
\|x+y\|^{p}+\|x-y\|^{p} \leq 2^{p-1} \cdot 2=2^{p} .
$$

Dividindo-se ambos os membros dessa equação por $2^{p}$ concluímos que

$$
\left\|\frac{x+y}{2}\right\|^{p}+\left\|\frac{x-y}{2}\right\|^{p} \leq 1 .
$$

Por $\left\|\frac{x+y}{2}\right\|=1$, devemos ter

$$
\left\|\frac{x-y}{2}\right\|=0
$$

daí segue que $x=y$ e isso conclui a demonstração neste caso.

Segundo Caso: $1<p \leq 2$. Pelo teorema 5 (d), temos:

$$
\|x+y\|^{q}+\|x-y\|^{q} \leq 2^{q-1} .2=2^{q} .
$$

Dividindo-se ambos os membros dessa equação por $2^{q}$ concluímos que

$$
\left\|\frac{x+y}{2}\right\|^{q}+\left\|\frac{x-y}{2}\right\|^{q} \leq 1
$$

Analogamente ao primeiro caso, concluímos que $x=y$. Isso conclui a demonstração do teorema, basta termos em mente a definição de espaços estritamente convexos. 


\section{Capítulo 2}

\section{Deslocamentos isométricos em $c \oplus_{\infty} X$}

\subsection{Introdução}

Neste capítulo, definiremos os deslocamentos isométricos em espaços de Banach quaisquer. Apesar da generalidade, estudá-los-emos, quase exclusivamente, em espaços da forma $c \oplus_{\infty} X$, sendo $X$ um espaço de Banach arbitrário e $c$ o espaço das sequências convergentes. Os lemas 3 e 4 e o teorema 10 descrevem um deslocamento isométrico definido em $c \oplus_{\infty} X$, quando $X$ for estritamente convexo. Esse último teorema mostra que a restrição do operador a $c$ é um novo deslocamento isométrico.

Os teoremas 8 e 9 são geradores de exemplos de deslocamentos em $c \oplus_{\infty} X$, após demonstrálos, daremos uma noção de como esses resultados podem ser úteis. A questão 1, apresentada na introdução da dissertação, está relacionada ao teorema 11, o qual é uma resposta para o problema no caso complexo. O caso real não foi resolvido neste trabalho e permanece em aberto.

A menos de menção contrária, consideraremos todo espaço vetorial arbitrário de dimensão infinita sobre um corpo $\mathbb{K}$, sendo $\mathbb{K}=\mathbb{R}$ ou $\mathbb{K}=\mathbb{C}$. O espaço produto estará sempre munido com a norma $\|\cdot\|_{\infty}$, a qual será explicada na próxima seção.

\subsection{Definição e algumas propriedades elementares dos desloca- mentos isométricos}

Nesta seção, vamos conhecer a definição de deslocamento isométrico e ver alguns resultados básicos. Esses resultados tratam do espaço produto entre dois espaços de Banach estritamente convexos, munido com a norma $\|\cdot\|_{\infty}$. Antes de apresentar a definição, vamos a um exemplo - talvez seja o exemplo mais conhecido de deslocamento em espaços de Banach.

Exemplo 1. Seja $T$ o operador definido por $\left(c_{0},\|\cdot\|_{\infty}\right) \stackrel{T}{\longrightarrow}\left(c_{0},\|\cdot\|_{\infty}\right)$ e pela relação:

$$
T\left(\left(a_{1}, a_{2}, \ldots, a_{n}, \ldots\right)\right)=\left(0, a_{1}, a_{2}, \ldots, a_{n}, \ldots\right)
$$


Nesse exemplo, verificamos sem dificuldades que Té uma isometria, o espaço vetorial ImT tem codimensão 1 e vale $\bigcap_{n=1}^{\infty} T^{n}(X)=\{0\}$.

Nossa intenção, a seguir, é generalizar operadores que satisfazem a essas propriedades.

Definição 12. Seja $X$ um espaço de Banach real ou complexo. Um operador $T$ em $X$ é chamado de deslocamento isométrico se satisfizer:

(1) T é uma isometria;

(2) O subespaço ImT tem codimensão 1;

(3) $\bigcap_{n=1}^{\infty} T^{n}(X)=\{0\}$.

Vamos observar outros casos notáveis:

Exemplo 2. Seja $p \in \mathbb{R}$ tal que $1 \leq p \leq \infty$. Seja $T$ o operador dado por

$$
\begin{array}{rlc}
T: & \ell_{p} & \ell_{p} \\
\left(a_{1}, a_{2}, \ldots, a_{n}, \ldots\right) & \longmapsto & \left(0, a_{1}, a_{2}, \ldots, a_{n}, \ldots\right)
\end{array}
$$

Vamos omitir a verificação de que Té um deslocamento isométrico, pois tal como no exemplo 1, é uma tarefa simples.

Antes de prosseguir, precisamos estar familiarizados com a norma $\|\cdot\|_{\infty}$, definida no espaço produto. Sejam $\left(X,\|\cdot\|_{X}\right)$ e $\left(Y,\|\cdot\|_{Y}\right)$ dois espaços de Banach. O espaço produto será representado por $X \oplus Y$. Consideremos a função $\|\cdot\|_{\infty}: X \oplus Y \longrightarrow \mathbb{R}_{+}$, dada por:

$$
\|(x, y)\|_{\infty}=\sup \left\{\|x\|_{X},\|y\|_{Y}\right\}, \text { para todos } x \in X \text { e } y \in Y \text {. }
$$

Podemos notar que $\|\cdot\|_{\infty}$ define uma norma em $X \oplus Y$, tornando $\left(X \oplus Y,\|\cdot\|_{\infty}\right)$ um espaço de Banach, o qual pode ser também denotado por $X \oplus_{\infty} Y$. Nesse caso, dizemos que o espaço produto está munido com a norma $\|\cdot\|_{\infty}$. Agora, vejamos alguns resultados básicos que podem ser encontrados em [5].

Lema 1. Sejam $X$ e $Y$ dois espaços normados estritamente convexos. Seja $T$ uma isometria de $X \oplus_{\infty} Y$ em $X \oplus_{\infty} Y$, então

$$
T(X) \subseteq Y \text { ou } T(X) \subseteq X
$$

Demonstração. Suponhamos que o lema não seja verdadeiro. Então existem $x^{\prime}, x^{\prime \prime} \in X$ de maneira que:

$$
T\left(\left(x^{\prime}, 0\right)\right)=\left(\alpha_{1}, \beta_{1}\right), T\left(\left(x^{\prime \prime}, 0\right)\right)=\left(\alpha_{2}, \beta_{2}\right), \text { com } \alpha_{1} \neq 0 \text { e } \beta_{2} \neq 0 .
$$

Daí existe $x \in X$ tal que $T((x, 0))=\left(x_{1}, y_{1}\right)$ com $x_{1} \neq 0$ e $y_{1} \neq 0$, a saber $x=x^{\prime}$ ou $x=x^{\prime \prime}$ ou $x=x^{\prime}+x^{\prime \prime}$ (ao menos uma dessas possibilidades satisfaz o que afirmamos). Sem perder generalidade, podemos supor que $\left\|x_{1}\right\|_{X} \geq\left\|y_{1}\right\|_{Y}$, daí como $T$ é isometria segue que

$$
\|x\|_{X}=\|(x, 0)\|_{\infty}=\|T(x, 0)\|_{\infty}=\left\|\left(x_{1}, y_{1}\right)\right\|_{\infty}=\left\|x_{1}\right\|_{X}
$$


Consideremos $y \in Y, y \neq 0$ qualquer. Digamos que $T((0, y))=\left(x_{2}, y_{2}\right)$, como $T$ é isometria temos $x_{2} \neq 0$ ou $y_{2} \neq 0$. Devemos tratar, separadamente, os dois casos que seguem:

Primeiro Caso. Suponhamos que $\left\|x_{2}\right\|_{X} \geq\left\|y_{2}\right\|_{Y}$. Então, por ser $T$ uma isometria, segue que

$$
\|y\|_{Y}=\|T(0, y)\|_{\infty}=\left\|\left(x_{2}, y_{2}\right)\right\|_{\infty}=\left\|x_{2}\right\|_{X}
$$

Neste caso podemos assumir $\|y\|_{Y} \leq\|x\|_{X}$, caso isso não seja verdade basta trocar o $y$ tomado inicialmente por $\frac{\|x\| y}{2\|y\|}$. Note que para todo $\theta \in \mathbb{K},|\theta|=1$, temos:

$$
\left\|x_{1}\right\|_{X}=\|x\|_{X}=\|(x, \theta y)\|_{\infty}=\|T(x, \theta y)\|_{\infty} \geq\left\|x_{1}+\theta x_{2}\right\|_{X} .
$$

Uma contradição com o corolário 2 , pois $X$ é estritamente convexo, $x_{1} \neq 0$ e $x_{2} \neq 0$.

Segundo Caso. Suponhamos que $\left\|y_{2}\right\|_{Y} \geq\left\|x_{2}\right\|_{X}$. Então, por ser $T$ uma isometria, segue que

$$
\|y\|_{Y}=\left\|y_{2}\right\|_{Y}
$$

Neste caso podemos assumir $\|y\|_{Y} \geq\|x\|_{X}$, caso isso não seja verdade basta trocar o $y$ tomado inicialmente por $\frac{2\|x\| y}{\|y\|}$. Note que para todo $\theta \in \mathbb{K},|\theta|=1$, temos:

$$
\left\|y_{2}\right\|_{Y}=\|y\|_{Y}=\|(x, \theta y)\|_{\infty}=\|T(x, \theta y)\|_{\infty} \geq\left\|y_{1}+\theta y_{2}\right\|_{Y} .
$$

Uma contradição com o corolário 2 , pois $Y$ é estritamente convexo, $y_{1} \neq 0$ e $y_{2} \neq 0$.

Assim provamos o lema pela redução ao absurdo.

O teorema 7 é útil para concluir que alguns espaços não admitem deslocamentos isométricos. Faremos esse tipo de uso do teorema 7 no capítulo 3.

Teorema 7. Sejam $X$ e $Y$ dois espaços de Banach estritamente convexos. Se $X \oplus_{\infty} Y$ admitir um deslocamento isométrico, então $X$ é isometricamente isomorfo a $Y$ e $X$ admite um deslocamento isométrico.

Demonstração. Suponhamos que $X \oplus_{\infty} Y$ admite um deslocamento isométrico $T$. Pelo lema 1, devemos ter $T(X) \subseteq X$ ou $T(X) \subseteq Y$. Vejamos que $T(X) \subseteq X$ não acontece:

Vamos admitir que $T(X) \subseteq X$. Pelo lema 1 , vale $T(Y) \subseteq X$ ou $T(Y) \subseteq Y$, mas $\operatorname{Im} T$ tem codimensão 1 e $Y$ é um subespaço de $X \oplus_{\infty} Y$ e de dimensão infinita, logo devemos ter $T(Y) \subseteq Y$. Agora notemos que, sendo $T$ um operador de codimensão 1, entre $T_{\left.\right|_{X}}: X \longrightarrow X$ e $T_{\left.\right|_{Y}}: Y \longrightarrow Y$, uma deve ser sobrejetora e a outra deve ter codimensão 1. Sem perder generalidade, suponhamos $T_{\left.\right|_{X}}: X \longrightarrow X$ sobrejetora. Portanto

$$
X \subseteq \bigcap_{n=1}^{\infty} T^{n}(X \oplus Y)
$$

Isso contradiz o fato de que $T$ é um deslocamento isométrico. Assim sabemos que necessariamente $T(X) \subseteq Y$. Por analogia ao raciocínio acima, segue que $T(Y) \subseteq X$. Novamente vem a ideia que entre $T_{\left.\right|_{X}}: X \longrightarrow Y$ e $T_{\left.\right|_{Y}}: Y \longrightarrow X$, uma deve ser sobrejetora e a outra deve ter codimensão 1. De qualquer maneira já temos que $X$ é isometricamente isomorfo a $Y$. 
Suponhamos, sem perda de generalidade que $T_{\left.\right|_{X}}: X \longrightarrow Y$ é sobrejetora. Vejamos que $\left(T^{2}\right)_{\left.\right|_{X}}$ é um deslocamento isométrico em $X$. Sabemos que

$$
\left(T^{2}\right)_{\left.\right|_{X}}=T_{\left.\right|_{Y}} \circ T_{\left.\right|_{X}}
$$

$\operatorname{logo}\left(T^{2}\right)_{\left.\right|_{X}}$ é uma isometria. Como $\operatorname{Im} T_{\left.\right|_{Y}} \neq X$, segue que $\operatorname{Im}\left(T^{2}\right)_{\left.\right|_{X}} \neq X$. Vamos provar que $\operatorname{Im}\left(T^{2}\right)_{\left.\right|_{X}}$ é um subespaço em $X$, de codimensão 1. Consideremos $\left(x_{1}, 0\right) \in X \backslash \operatorname{Im}\left(T^{2}\right)_{\left.\right|_{X}}$, vejamos que $\left(x_{1}, 0\right) \notin \operatorname{Im} T$. De fato, se $\left(x_{1}, 0\right)=T\left(x_{2}, y_{2}\right)$, como $T\left(x_{2}, 0\right) \in Y$ e $T$ é isometria, segue que $x_{2}=0$. $\operatorname{Logo}\left(x_{1}, 0\right)=T\left(0, y_{2}\right)$. Mas $T_{\left.\right|_{X}}: X \longrightarrow Y$ é sobrejetora e assim $\left(x_{1}, 0\right) \in$ $\operatorname{Im}\left(T^{2}\right)_{\left.\right|_{X}}$, contra nossa hipótese. Portanto $\left(x_{1}, 0\right) \notin \operatorname{Im} T$.

Sabemos que $\left(x_{1}, 0\right) \notin \operatorname{ImT}$ e $T$ é um operador de codimensão 1, isso implica que

$$
X \oplus Y=\mathbb{K}\left(x_{1}, 0\right) \oplus I m T .
$$

Logo, para todo $x \in X$, existem $\lambda_{x} \in \mathbb{K}$ e $\left(\alpha_{x}, \beta_{x}\right) \in X \oplus Y$ tais que

$$
(x, 0)=\lambda_{x}\left(x_{1}, 0\right)+T\left(\alpha_{x}, \beta_{x}\right) .
$$

Como $T\left(\alpha_{x}, 0\right) \in Y$ e $T$ é uma isometria, segue que $\alpha_{x}=0$. Então $(x, 0)=\lambda_{x}\left(x_{1}, 0\right)+T\left(0, \beta_{x}\right)$ e, por $T_{\left.\right|_{X}}: X \longrightarrow Y$ ser sobrejetora, $\left(0, \beta_{x}\right) \in \operatorname{Im} T_{\left.\right|_{X}}$. Segue que $\left((x, 0)-\lambda_{x}\left(x_{1}, 0\right)\right) \in \operatorname{Im}\left(T^{2}\right)_{\left.\right|_{X}}$ e com isso provamos que

$$
X=\mathbb{K}\left(x_{1}, 0\right) \oplus \operatorname{Im}\left(T^{2}\right)_{\left.\right|_{X}} .
$$

Pela generalidade envolvida, podemos concluir que $\operatorname{Im}\left(T^{2}\right)_{\left.\right|_{X}}$ é um subespaço de codimensão 1. Resta-nos apenas demonstrar que $\bigcap_{n=1}^{\infty} \operatorname{Im}\left(\left(T^{2}\right)_{\mid X}\right)^{n}=\{0\}$, isso segue diretamente do fato que:

$$
\operatorname{Im} T \supseteq \operatorname{Im} T^{2} \supseteq \ldots \supseteq \operatorname{Im} T^{n} \supseteq \ldots
$$

Com isso, provamos que $\left(T^{2}\right)_{\left.\right|_{X}}$ é um deslocamento isométrico em $X$. Como $Y$ é isometricamente isomorfo a $X$, concluímos que $Y$ também admite um deslocamento isométrico.

\subsection{Construção de deslocamentos isométricos}

Esta seção é dedicada a construção de deslocamentos isométricos no espaço produto, munido com a norma $\|\cdot\|_{\infty}$. Com os teoremas 8 e 9 , seremos capazes de construir exemplos bem interessantes de deslocamentos em $c \oplus_{\infty} X$, como é o caso do exemplo 4. Todos os resultados e exemplos presentes nesta seção podem ser vistos em [5]. O exemplo 3 abrirá espaço para enunciar esses dois teoremas.

Exemplo 3. Seja $X$ um espaço de Banach e $S$ uma isometria sobrejetora de $X$ em $X$. Seja $x^{*}$ um elemento da bola unitária de $X^{*}$. Definimos

$$
\begin{aligned}
T_{S, x^{*}}: c \oplus_{\infty} X & \longrightarrow c \oplus_{\infty} X \\
\left(\left(a_{n}\right)_{n}, x\right) & \longmapsto\left(\left(b_{n}\right)_{n}, S(x)\right)
\end{aligned}
$$


de modo que:

$$
b_{n}=\left\{\begin{array}{rrr}
x^{*}(x), & \text { se } & n=1 \\
a_{n}, & \text { se } & n>1
\end{array}\right.
$$

Obviamente $T_{S, x^{*}}$ é uma aplicação linear, vejamos que $T_{S, x^{*}}$ é uma isometria. De fato, para $\left(\left(a_{n}\right)_{n}, x\right) \in c \oplus_{\infty} X$ qualquer, temos:

$$
\begin{aligned}
\left.\| T_{S, x^{*}}\left(\left(a_{n}\right)_{n}\right), x\right) \|_{\infty} & =\sup \left\{\|S(x)\|,\left\|\left(b_{n}\right)_{n}\right\|_{c}\right\}=\sup \left\{\|S(x)\|,\left|x^{*}(x)\right|,\left\|\left(a_{n}\right)_{n}\right\|_{c}\right\}= \\
& =\sup \left\{\|x\|,\left|x^{*}(x)\right|,\left\|\left(a_{n}\right)_{n}\right\|_{c}\right\} \\
& =\sup \left\{\|x\|,\left\|\left(a_{n}\right)_{n}\right\|_{c}\right\} \\
& \left.=\|\left(\left(a_{n}\right)_{n}\right), x\right) \|_{\infty} .
\end{aligned}
$$

Acima, usamos que $\|S(x)\|=\|x\| e\left\|x^{*}(x)\right\| \leq\|x\|$. Logo $T_{S, x^{*}}$ é uma isometria. Agora, mostremos que $T_{S, x^{*}}$ é um operador de codimensão 1. Notemos que:

$$
\operatorname{Im} T_{S, x^{*}}=\left\{\left(\left(a_{n}\right)_{n}, y\right): a_{1}=x^{*}\left(S^{-1}(y)\right)\right\}
$$

Assim, é evidente que $T_{S, x^{*}}$ não é sobrejetora. Suponhamos $\left(\left(b_{n}\right)_{n}, z\right) \in c \oplus_{\infty} X$, de modo que $\left(\left(b_{n}\right)_{n}, z\right) \notin I m T_{S, x^{*}}$. Então já sabemos que $b_{1} \neq x^{*}\left(S^{-1}(z)\right)$, dado qualquer $\left(\left(c_{n}\right)_{n}, w\right) \in c \oplus_{\infty} X$, consideremos

$$
\lambda=\frac{c_{1}-x^{*}\left(S^{-1}(w)\right)}{b_{1}-x^{*}\left(S^{-1}(z)\right)} .
$$

Pela igualdade (2.1), verificamos que $\left(\left(c_{n}\right)_{n}, w\right)-\lambda\left(\left(b_{n}\right)_{n}, z\right) \in I m T_{S, x^{*}}$. Assim, provamos que $T_{S, x^{*}}$ é um operador de codimensão 1.

Observação 2. No exemplo 3, é evidente que $T_{S, x^{*}}\left(c_{0} \oplus X\right) \subseteq c_{0} \oplus X$. Além disso, concluímos que $T_{S, x^{*}}$ é um deslocamento isométrico em $c \oplus_{\infty} X$ se, e somente se, $\bigcap_{n=1}^{\infty} T_{S, x^{*}}^{n}(c \oplus X)=\{0\}$.

Teorema 8. Seja X um espaço de Banach e $S$ uma isometria sobrejetora de $X$ em X. Seja $x^{*}$ um elemento da bola unitária de $X^{*}$. Então $T_{S, x^{*}}$ é um deslocamento isométrico em $c \oplus_{\infty} X$ se, e somente se, para cada $x \in X$, a existência do limite

$$
\lim _{n \rightarrow \infty} x^{*}\left(S^{-n}(x)\right)
$$

implica que $x=0$.

Demonstração. Vamos provar primeiro que para cada $k \in \mathbb{N}$, vale:

Se $\left(\left(a_{n}\right)_{n}, x\right) \in I m T_{S, x^{*}}^{k}$, então $a_{j}=x^{*}\left(S^{-j}(x)\right)$, para todo $j \in\{1,2, \ldots, k\}$.

Façamos isso através de uma indução em $k$. Para $k=1$, o resultado segue da definição do operador. Vamos supor que:

$$
\text { Se }\left(\left(a_{n}\right)_{n}, x\right) \in I m T_{S, x^{*}}^{k} \text {, então } a_{j}=x^{*}\left(S^{-j}(x)\right), \forall j \in\{1,2, \ldots, k\}
$$

para algum $k \in \mathbb{N}$ arbitrário e consideremos $\left(\left(a_{n}\right)_{n}, x\right) \in \operatorname{Im}_{S, x^{*}}^{k+1}$. Então existe $\left(\left(b_{n}\right)_{n}, y\right) \in$ $c \oplus_{\infty} X$ tal que $\left(\left(a_{n}\right)_{n}, x\right)=T_{S, x^{*}}^{k+1}\left(\left(\left(b_{n}\right)_{n}, y\right)\right)=T_{S, x^{*}}\left(T_{S, x^{*}}^{k}\left(\left(\left(b_{n}\right)_{n}, y\right)\right)\right)$. Seja $\left(\left(c_{n}\right)_{n}, w\right)=$ 
$T_{S, x^{*}}^{k}\left(\left(\left(b_{n}\right)_{n}, y\right)\right)$. Portanto $\left(\left(a_{n}\right)_{n}, x\right)=T_{S, x^{*}}\left(\left(\left(c_{n}\right)_{n}, w\right)\right)$ e $\left(\left(c_{n}\right)_{n}, w\right) \in \operatorname{Im} T_{S, x^{*}}^{k}$. Aplicando a hipótese de indução para $\left(\left(c_{n}\right)_{n}, w\right)$, concluímos que $c_{j}=x^{*}\left(S^{-j}(w)\right)$, para cada $j \in\{1,2, \ldots, k\}$. Pela definição do operador $T_{S, x^{*}}$, sabemos que

$$
a_{1}=x^{*}(w), a_{2}=c_{1}=x^{*}\left(S^{-1}(w)\right), a_{3}=c_{2}=x^{*}\left(S^{-2}(w)\right), \ldots, a_{k+1}=c_{k}=x^{*}\left(S^{-k}(w)\right) .
$$

Por fim, notemos que $x=S(w)$, $\log o w=S^{-1}(x)$. Substituindo esse fato nas igualdades acima, acarreta que $a_{j}=x^{*}\left(S^{-j}(x)\right)$ para todo $j \in\{1,2, \ldots, k, k+1\}$, então o resultado vale para $k+1$ e está provado por indução.

Vejamos agora que vale a recíproca do resultado - se $\left(\left(a_{n}\right)_{n}, x\right) \in c \oplus_{\infty} X$ é tal que

$$
a_{j}=x^{*}\left(S^{-j}(x)\right), \forall j \in\{1,2, \ldots, k\},
$$

então $\left(\left(a_{n}\right)_{n}, x\right) \in \operatorname{Im} T_{S, x^{*}}^{k}$. Para tal, basta definir $\left(\left(b_{n}\right)_{n}, y\right) \in c \oplus_{\infty} X$ da seguinte forma:

$$
y=S^{-k}(x), b_{1}=a_{k+1}, b_{2}=a_{k+2}, \ldots, b_{l}=a_{k+l}, \forall l \in \mathbb{N} .
$$

Uma fácil verificação mostra que $\left(\left(a_{n}\right)_{n}, x\right)=T_{S, x^{*}}^{k}\left(\left(b_{n}\right)_{n}, y\right)$ e assim vale também a recíproca.

Dessa forma, consideremos $\left(\left(a_{n}\right)_{n}, x\right) \in c \oplus X$. Provamos que, para todo $k \in \mathbb{N}$, vale

$$
\left(\left(a_{n}\right)_{n}, x\right) \in \operatorname{Im} T_{S, x^{*}}^{k} \text { se, e somente se, } a_{j}=x^{*}\left(S^{-j}(x)\right) \text { para todo } j \in\{1,2, \ldots, k\} .
$$

Queremos provar agora que $T_{S, x^{*}}$ é um deslocamento isométrico em $c \oplus_{\infty} X$ se, e somente se,

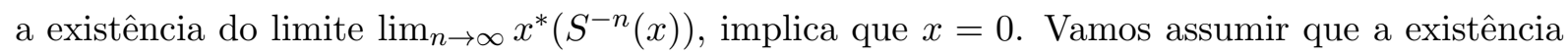
do limite

$$
\lim _{n \rightarrow \infty} x^{*}\left(S^{-n}(x)\right),
$$

implica que $x=0$. Notemos que, se $\left(\left(a_{n}\right)_{n}, x\right) \in \bigcap_{n=1}^{\infty} T_{S, x^{*}}^{n}(c \oplus X)$, o resultado (2.2) diz que

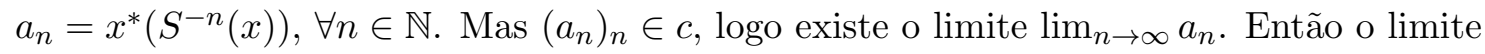

$$
\lim _{n \rightarrow \infty} x^{*}\left(S^{-n}(x)\right)
$$

existe. Logo, pelo que assumimos, $x=0$. Assim temos $a_{n}=x^{*}\left(S^{-n}(0)\right)=0$, para todo $n \in \mathbb{N}$. Com isso $\left(\left(a_{n}\right)_{n}, x\right)=\left((0)_{n}, 0\right)$. Através da generalidade e da observação 2 , concluímos que $T_{S, x^{*}}$ é um deslocamento isométrico.

Por outro lado, vamos assumir que $T_{S, x^{*}}$ é um deslocamento isométrico em $c \oplus_{\infty} X$ e supor

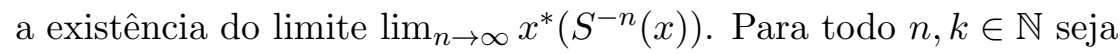

$$
a_{k}^{n}=x^{*}\left(S^{-n-k}(x)\right) .
$$

Portanto $\left(a_{k}^{n}\right)_{k} \in c$ para cada $n \in \mathbb{N}$. Pela definição de $T_{S, x^{*}}$, vem que $T_{S, x^{*}}^{n}\left(\left(a_{k}^{n}\right)_{k}, S^{-n}(x)\right)=$ $\left(\left(a_{k-1}^{1}\right)_{k}, x\right)$, para todo $n \in \mathbb{N}$. Assim $\left(\left(a_{k-1}^{1}\right)_{k}, x\right) \in \bigcap_{n=1}^{\infty} T_{S, x^{*}}^{n}(c \oplus X)$, mas como $T_{S, x^{*}}$ é um deslocamento isométrico, devemos ter $x=0$ e isso conclui a prova.

O teorema a seguir pode ser demonstrado de maneira análoga ao teorema 8, basta ter em mente a observação 2. Dessa maneira, enunciaremos o próximo teorema sem o demonstrar. 
Teorema 9. Seja $X$ um espaço de Banach e $S$ uma isometria sobrejetora de $X$ em $X$. Seja $x^{*}$ um elemento da bola unitária de $X^{*}$. Então $T_{S, x^{*}}$ é um deslocamento isométrico em $c_{0} \oplus_{\infty} X$ se, e somente se, para cada $x \in X$, quando

$$
\lim _{n \rightarrow \infty} x^{*}\left(S^{-n}(x)\right)=0
$$

necessariamente temos $x=0$.

O próximo lema ajudará no exemplo 4. Esse lema é um resultado bem simples de topologia. A notação $\mathbb{C}_{\infty}^{n}$ indica o espaço produto $\mathbb{C}^{n}$, munido com a norma $\|\cdot\|_{\infty}$.

Lema 2. Existe um conjunto $A \subseteq \bigcup_{n=1}^{\infty} \mathbb{C}_{\infty}^{n}$ satisfazendo as seguintes condições:

(1) A é enumerável;

(2) $\|z\|_{\infty}=1$, para todo $z \in A$;

(3) Dados quaisquer $n \in \mathbb{N}$ e $w \in \mathbb{C}_{\infty}^{n}$ com $\|w\|_{\infty}=1$, existe $z \in A \cap \mathbb{C}_{\infty}^{n}$ de modo que $\|w-z\|_{\infty}<\frac{1}{6}$.

Demonstração. Vamos fixar $n \in \mathbb{N}$ e considerar $r=\frac{1}{6}$. Evidentemente a família

$$
\mathcal{F}=\left\{B(z ; r): z \in \mathbb{C}_{\infty}^{n} \mathrm{e}\|z\|_{\infty}=1\right\}
$$

é uma cobertura por abertos do conjunto $\left\{z \in \mathbb{C}_{\infty}^{n}:\|z\|_{\infty}=1\right\}$. Como esse conjunto é compacto, admite uma subcobertura finita. Logo existe $\left\{B\left(z_{1} ; r\right), B\left(z_{2} ; r\right), \ldots, B\left(z_{l} ; r\right)\right\} \subseteq \mathcal{F}$ de modo que

$$
\left\{z \in \mathbb{C}_{\infty}^{n}:\|z\|_{\infty}=1\right\} \subseteq \bigcup_{j=1}^{l} B\left(z_{j} ; r\right) .
$$

Consideremos $A_{n}=\left\{z_{1}, z_{2}, \ldots, z_{l}\right\}$. Ao fazer esse raciocínio para todo $n \in \mathbb{N}$, construímos um conjunto

$$
A=\bigcup_{n \in \mathbb{N}} A_{n}
$$

É fácil ver que esse conjunto satisfaz (1), (2) e (3).

Observação 3. Vamos enumerar o conjunto A do lema 2:

$$
A=\left\{z_{k}: k \in \mathbb{N}\right\}
$$

Com isso, podemos exigir também que o conjunto A satisfaça a seguinte condição:

(4) Para dois naturais $k_{1}, k_{2}$ quaisquer de modo que $k_{1} \leq k_{2}$, $z_{k_{1}} \in \mathbb{C}_{\infty}^{n}$ e $z_{k_{2}} \in \mathbb{C}_{\infty}^{m}$, temos $n \leq m$.

Por fim, encerramos esta seção com uma aplicação dos teoremas 8 e 9. 
Exemplo 4. Consideremos o espaço de Banach $X=\ell_{1}(\mathbb{Z})$ sobre o corpo $\mathbb{K}=\mathbb{C}$. Seja $S$ a isometria definida por :

$$
\begin{aligned}
S: X & \longrightarrow X \\
\left(x_{n}\right) & \longmapsto\left(y_{n}\right)
\end{aligned}
$$

com $y_{n}=x_{n-1}$, para todo $n \in \mathbb{N}$. Através da definição observamos que, para um $k \in \mathbb{N}$ arbitrário, valem:

i) $S^{k}\left(\left(x_{n}\right)\right)=\left(y_{n}\right)$ com $y_{n}=x_{n-k}$, para todo $n \in \mathbb{N}$;

ii) $S^{-k}\left(\left(x_{n}\right)\right)=\left(y_{n}\right)$ com $y_{n}=x_{n+k}$, para todo $n \in \mathbb{N}$.

Consideremos $A=\left\{z_{k}: k \in \mathbb{N}\right\}$, um conjunto como no enunciado do lema 2 e de acordo com a observação 3. Vamos definir $\left(d_{n}\right)_{n} \in \ell_{\infty}(\mathbb{Z})$ da seguinte forma:

$$
d_{n}=0, \text { para cada } n \geq 0 .
$$

Suponhamos $z_{1}=\left(a_{1}, a_{2}, \ldots, a_{j}\right) \in \mathbb{C}_{\infty}^{j}$, definimos $d_{-i}=a_{i}$ para todo $i \in\{1,2, \ldots, j\}$. Suponhamos $N<0$ o menor índice para o qual $d_{n}$ está definido e que o processo acima foi repetido para $z_{1}, z_{2}, \ldots, z_{k}$ ( $k$ vezes). Daí, se $z_{k+1}=\left(b_{1}, b_{2}, \ldots, b_{l}\right) \in \mathbb{C}_{\infty}^{l}$, fazemos: $d_{N-i}=b_{i}$ para todo $i \in\{1,2, \ldots, l\}$. Temos assim $\left(d_{n}\right)_{n} \in \ell_{\infty}(\mathbb{Z})$ definido por indução. Observemos que $\left\|d_{n}\right\|_{\infty} \leq 1$ $e\left(d_{n}\right)_{n}$ pode ser identificado com $x^{*} \in \ell_{1}^{*}(\mathbb{Z})$ da seguinte forma:

$$
x^{*}\left(\left(a_{n}\right)_{n}\right)=\sum_{n \in \mathbb{Z}} a_{n} d_{n} \in \mathbb{C},
$$

para todo $\left(a_{n}\right)_{n} \in \ell_{1}(\mathbb{Z})$. Sabemos ainda que esse funcional satisfaz $\left\|x^{*}\right\|_{X^{*}} \leq 1$.

Vamos mostrar que, para todo $x \in X$ e para todo $N \in \mathbb{N}$, existem $N_{1}, N_{2}$ naturais tais que $N_{1}>N, N_{2}>N$ e:

$$
\mathcal{R}\left(x^{*}\left(S^{-N_{1}}(x)\right)\right) \leq-\frac{\|x\|_{1}}{2} \leq \frac{\|x\|_{1}}{2} \leq \mathcal{R}\left(x^{*}\left(S^{-N_{2}}(x)\right)\right) .
$$

Vamos mostrar apenas que $\frac{\|x\|_{1}}{2} \leq \mathcal{R}\left(x^{*}\left(S^{-N_{2}}(x)\right)\right)$ e comentar como obter a outra desigualdade. Sendo assim, considere $x=\left(x_{n}\right) \in X, x \neq 0$ e $N \in \mathbb{N}$ arbitrários. Seja $\varepsilon=\frac{\|x\|_{1}}{6}>0$. Segue que existe $F \subseteq \mathbb{Z}$ finito de modo que

$$
\sum_{n \in \mathbb{Z}}\left|x_{n}\right|-\sum_{n \in F}\left|x_{n}\right|<\varepsilon
$$

Notemos ainda que, todo conjunto $F^{\prime}$ de inteiros tal que $F \subseteq F^{\prime}$, satisfaz

$$
\sum_{n \in \mathbb{Z}}\left|x_{n}\right|-\sum_{n \in F^{\prime}}\left|x_{n}\right|<\varepsilon
$$

Por esse motivo, podemos supor $F=\{c, c-1, \ldots, c-l\} \subseteq \mathbb{Z}$ finito satisfazendo (2.4), para algum natural $l$.

Podemos supor, sem perder generalidade, que todo indice $n \in \mathbb{Z}$ dos $d_{n^{\prime} s}$ correspondentes aos $z_{k^{\prime} s} \in \mathbb{C}_{\infty}^{l+1}$, verifica $c-n>N$. Vejamos que se isso não acontece, existe $t \in \mathbb{N}, t>l$, tal que 
essa afirmação é válida para t.

Sabemos que existem $w_{0}, w_{1}, \ldots, w_{l} \in \mathbb{C}$ de módulo 1 tais que $w_{i} x_{c-i}=\left|x_{c-i}\right|$, para todo $i \in\{0,1, \ldots, l\}$. Considere $\left(w_{l}, w_{l-1}, \ldots, w_{1}, w_{0}\right) \in \mathbb{C}_{\infty}^{l+1}$, dai existe $m \in \mathbb{Z}, m<0$ tal que

$$
\left\|\left(d_{m-l}, \ldots, d_{m-1}, d_{m}\right)-\left(w_{l}, \ldots, w_{1}, w_{0}\right)\right\|_{\infty}<\frac{1}{6} .
$$

Pelo que estamos supondo, $c-m>N$. Sejam $N_{2}=c-m>N$ e $S^{-N_{2}}\left(\left(x_{n}\right)\right)=\left(y_{n}\right)$, então $y_{n}=x_{N_{2}+n}$, para todo $n \in \mathbb{N}$. Portanto temos:

$$
y_{m}=x_{c}, y_{m-1}=x_{c-1}, \ldots, y_{m-l}=x_{c-l} .
$$

Temos $x^{*}\left(S^{-N_{2}}(x)\right)=\sum_{n \in \mathbb{Z}}\left(d_{n} y_{n}\right)$. Considere $I=\{n \in \mathbb{Z}: m-l \leq n \leq m\}$, dai:

$$
\begin{aligned}
\left|\|x\|_{1}-\mathcal{R}\left(\sum_{n \in \mathbb{Z}} d_{n} y_{n}\right)\right|=\left|\|x\|_{1}-\mathcal{R}\left(\sum_{n \in I} d_{n} y_{n}\right)-\mathcal{R}\left(\sum_{n \in \mathbb{Z} \backslash I} d_{n} y_{n}\right)\right| \leq A+B, \\
\operatorname{para} A=\left|\|x\|_{1}-\mathcal{R}\left(\sum_{n \in I} d_{n} y_{n}\right)\right| e B=\left|\mathcal{R}\left(\sum_{n \in \mathbb{Z} \backslash I} d_{n} y_{n}\right)\right| . \\
A=\left|\|x\|_{1}-\mathcal{R}\left(\sum_{n \in I} d_{n} y_{n}\right)\right|=\left|\|x\|_{1}-\mathcal{R}\left(d_{m} x_{c}+d_{m-1} x_{c-1}+\ldots+d_{m-l} x_{c-l}\right)\right|= \\
=\left|\|x\|_{1}-\mathcal{R}\left(\sum_{i=0}^{i=l} w_{i} x_{c-i}\right)+\mathcal{R}\left(\sum_{i=0}^{i=l} w_{i} x_{c-i}\right)-\mathcal{R}\left(\sum_{i=0}^{i=l} d_{m-i} x_{c-i}\right)\right| \leq \\
\leq\left|\|x\|_{1}-\sum_{i=0}^{l}\right| x_{c-i}||+\left|\mathcal{R}\left(\sum_{i=0}^{l} x_{c-i}\left(w_{i}-d_{m-i}\right)\right)\right|< \\
<\varepsilon+\frac{1}{6} \sum_{i=0}^{l}\left|x_{c-i}\right| \leq \varepsilon+\frac{1}{6}\|x\|_{1}=2 \varepsilon .
\end{aligned}
$$

Temos ainda:

$$
\begin{aligned}
B & =\left|\mathcal{R}\left(\sum_{n \in \mathbb{Z} \backslash I} d_{n} y_{n}\right)\right| \leq\left|\sum_{n \in \mathbb{Z} \backslash I} d_{n} y_{n}\right| \leq\left\|x^{*}\right\| \sum_{n \in \mathbb{Z} \backslash I}\left|y_{n}\right| \leq \\
& \leq \sum_{n \in \mathbb{Z} \backslash I}\left|y_{n}\right|=\sum_{n \notin\{c, c-1, \ldots, c-l\}}\left|x_{n}\right|<\varepsilon .
\end{aligned}
$$

Assim $\left|\|x\|_{1}-\mathcal{R}\left(x^{*}\left(S^{-N_{2}}(x)\right)\right)\right| \leq A+B<3 \varepsilon=\frac{\|x\|_{1}}{2}$. Então temos $N_{2}>N$ e:

$$
\frac{\|x\|_{1}}{2} \leq \mathcal{R}\left(x^{*}\left(S^{-N_{2}}(x)\right)\right) .
$$

Vejamos uma ideia de como obter $\mathcal{R}\left(x^{*}\left(S^{-N_{1}}(x)\right)\right) \leq-\frac{\|x\|_{1}}{2}$ para algum $N_{1} \in \mathbb{N}, N_{1}>N$. Sejam $x=\left(x_{n}\right) \in X, x \neq 0$ e $N \in \mathbb{N}$ arbitrários. Consideremos $\varepsilon=\frac{\|x\|_{1}}{6}>0$ e $F=$ 
$\{c, c-1, \ldots, c-l\} \subseteq \mathbb{Z}$, como em (2.4). Novamente, suponhamos que todo indice $n \in \mathbb{Z}$ dos $d_{n^{\prime}}$ correspondentes aos $z_{k^{\prime} s} \in \mathbb{C}_{\infty}^{l+1}$, verifica $c-n>N$. Daí existem $w_{0}, w_{1}, \ldots, w_{l} \in \mathbb{C}$ de módulo 1 tais que $w_{i} x_{c-i}=-\left|x_{c-i}\right|$, para todo $i \in\{0,1, \ldots, l\}$. Considere $\left(w_{l}, w_{l-1}, \ldots, w_{1}, w_{0}\right) \in \mathbb{C}_{\infty}^{l+1}$, dai existe $m \in \mathbb{Z}, m<0$ tal que

$$
\left\|\left(d_{m-l}, \ldots, d_{m-1}, d_{m}\right)-\left(w_{l}, \ldots, w_{1}, w_{0}\right)\right\|_{\infty}<\frac{1}{6} .
$$

Daí, seguindo analogamente a primeira parte, obtemos:

$$
\left|-\|x\|_{1}-\mathcal{R}\left(x^{*}\left(S^{-N_{1}}(x)\right)\right)\right| \leq \frac{\|x\|_{1}}{2}
$$

donde tiramos que $\mathcal{R}\left(x^{*}\left(S^{-N_{1}}(x)\right)\right) \leq-\frac{\|x\|_{1}}{2}$. Então, provamos as desigualdades em (2.3). Dessa forma para um $N \in \mathbb{N}$ arbitrário, existem $N_{1}, N_{2} \in \mathbb{N}$, maiores que $N$, de modo que:

$$
0 \leq\|x\|_{1}=\frac{\|x\|_{1}}{2}-\left(-\frac{\|x\|_{1}}{2}\right) \leq \mathcal{R}\left(x^{*}\left(S^{-N_{2}}(x)\right)\right)-\mathcal{R}\left(x^{*}\left(S^{-N_{1}}(x)\right)\right) .
$$

Notemos que essas desigualdades são estabelecidas para cada $x \in X$, apenas variando os naturais $N_{1}$ e $N_{2}$. Logo se $x \in X$ e existe $\lim _{n \rightarrow \infty} x^{*}\left(S^{-n}(x)\right)$, devemos ter $x=0$. Pelos teoremas 8 e 9, $c \oplus_{\infty} \ell_{1}$ e $c_{0} \oplus_{\infty} \ell_{1}$ admitem um deslocamento isométrico.

\subsection{Deslocamentos isométricos em $c \oplus_{\infty} X$}

Nesta seção, descreveremos um deslocamento isométrico em $c \oplus_{\infty} X$, quando $X$ for estritamente convexo. Nesse contexto, o lema 3 não apenas trata de deslocamentos, mas de qualquer isometria. Já o lema 4, explora qualquer isometria cuja imagem tenha codimensão 1. O teorema 10 provará que se $T$ é um deslocamento isométrico definido em $c \oplus_{\infty} X$, então a restrição de $T$ a $c$ é um novo deslocamento isométrico. Esses três resultados podem ser encontrados em [5].

O teorema 11 é uma solução do problema da questão 1, no caso complexo. Após demonstrá-lo, comentaremos sobre seus desdobramentos, os quais envolvem muitos exemplos de deslocamentos. Esse teorema foi estudado em [3].

Lema 3. Seja $X$ um espaço de Banach estritamente convexo e de dimensão infinita e seja $Q$ a projeção natural de $c \oplus_{\infty} X$ em $X$. Suponhamos que $T$ seja uma isometria, definida por $T: c \oplus_{\infty} X \longrightarrow c \oplus_{\infty} X$. Então:

(1) $T(c) \subseteq c$;

(2) $Q \circ T_{\left.\right|_{X}}$ é uma isometria.

Demonstração. Antes de mostrarmos (1), vamos observar alguns fatos. Como $X$ é um espaço de Banach de dimensão infinita, existe $x \in X, x \neq 0$ tal que $T((0, x))=\left(y_{1}, x_{1}\right)$, com $y_{1} \in c_{0}$. De fato, basta tomar $\left\{x_{a}, x_{b}\right\} \subseteq X$ linearmente independente. Digamos que

$$
T\left(\left(0, x_{a}\right)\right)=\left(y_{a}, z_{a}\right), T\left(\left(0, x_{b}\right)\right)=\left(y_{b}, z_{b}\right) \operatorname{com} y_{a} \rightarrow \alpha \text { e } y_{b} \rightarrow \beta .
$$


Daí para $\alpha=0$, tomamos $x=x_{a}$. Para $\beta=0$, tomamos $x=x_{b}$ e quando $\alpha \neq 0$ e $\beta \neq 0$, tomamos $x=x_{a}-\frac{\alpha}{\beta} x_{b}$. Já que garantimos a existência, fixemos $x$ como acima.

Afirmo que $\|x\|_{X}=\left\|x_{1}\right\|_{X}$. Suponhamos, por absurdo, $\|x\|_{X}-\left\|x_{1}\right\|_{X}=\varepsilon>0$. Seja $y_{1}=\left(a_{n}\right)_{n}, \log 0\|x\|_{X}=\left\|y_{1}\right\|_{c}$. Considere

$$
A=\left\{k \in \mathbb{N}:\left|a_{k}\right| \geq\left\|y_{1}\right\|_{c}-\frac{\varepsilon}{2}\right\} .
$$

Como $y_{1} \in c_{0}, A$ deve ser finito. Vamos construir $z \in X, z \neq 0$ que satisfaz $T((0, z))=\left(\left(b_{n}\right)_{n}, x_{3}\right)$ e $b_{k}=0$ para todo $k \in A$. Para tal, suponhamos $A=\left\{k_{1}, k_{2}, \ldots, k_{l}\right\}$, ou seja, $|A|=l \in \mathbb{N}$. Sejam $w_{1}, w_{2}, \ldots, w_{l+1}$ vetores linearmente independentes em $X$ e $T\left(w_{i}\right)=\left(\left(\alpha_{n}^{i}\right)_{n}, \beta_{i}\right)$, para cada $i \in\{1,2, \ldots, l+1\}$. Consideremos o seguinte sistema:

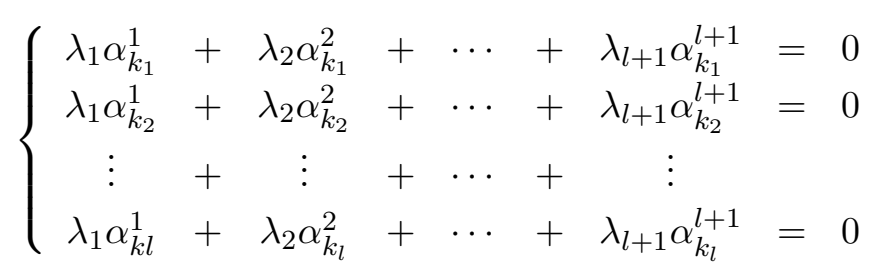

Esse sistema tem $l$ equações e $l+1$ incógnitas, logo admite uma solução não trivial, digamos $\left(\delta_{1}, \delta_{2}, \cdots, \delta_{l+1}\right)$. Observe daí que $z=\sum_{i=1}^{l+1} \delta_{i} w_{i}$ verifica o que gostaríamos. Ainda podemos supor $\|z\|_{X} \leq \frac{\varepsilon}{2}$, pois se isso não for verdade basta trocar $z$ por $\frac{\varepsilon}{2\|z\|} z$.

Assim, para todo $\theta \in \mathbb{K} \operatorname{com}|\theta|=1$, temos:

$$
\begin{aligned}
\|x+\theta z\|_{X} & =\|T(0, x+\theta z)\|_{\infty}=\left\|\left(\left(a_{n}\right), x_{1}\right)+\theta\left(\left(b_{n}\right), x_{3}\right)\right\|_{\infty} \leq \\
& \leq \sup \left(\left\{\left|a_{n}\right|: n \in A\right\} \cup\left\{\left|a_{n}+\theta b_{n}\right|: n \notin A\right\} \cup\left\{\left\|x_{1}+\theta x_{3}\right\|_{X}\right\}\right) .
\end{aligned}
$$

Notemos que:

$\sup \left(\left\{\left|a_{n}\right|: n \in A\right\}\right) \leq\left\|y_{1}\right\|_{c}=\|x\|_{X}$

Se $n \notin A,\left|a_{n}+\theta b_{n}\right| \leq\left|a_{n}\right|+\left|\theta b_{n}\right| \leq\left\|y_{1}\right\|_{c}-\frac{\varepsilon}{2}+|\theta|\|z\|_{X} \leq\left\|y_{1}\right\|_{c}-\frac{\varepsilon}{2}+\frac{\varepsilon}{2}=\left\|y_{1}\right\|_{c}=\|x\|_{X}$, aqui usamos que $\left|a_{n}\right| \leq\left\|y_{1}\right\|_{c}-\frac{\varepsilon}{2}$ se $n \notin A,|\theta|=1$ e $\|z\|_{X} \leq \frac{\varepsilon}{2}$;

$\left\|x_{1}+\theta x_{3}\right\|_{X} \leq\left\|x_{1}\right\|+|\theta|\left\|x_{3}\right\| \leq\left\|x_{1}\right\|+\frac{\varepsilon}{2} \leq\|x\|_{X}$, lembrando que $\|x\|_{X}-\left\|x_{1}\right\|_{X}=\varepsilon$.

Portanto $\|x+\theta z\|_{X} \leq\|x\|_{X}$ para todo $\theta \in \mathbb{K}$ de módulo 1, uma contradição com o corolário 2, pois $X$ é estritamente convexo, $x \neq 0$ e $z \neq 0$. Provamos então que $\|x\|_{X}=\left\|x_{1}\right\|_{X}$, pela redução ao absurdo.

Mostremos (1). Para tal, seja $y \in c, y \neq 0$ arbitrário. Seja $\bar{y}=\frac{\|x\|}{2\|y\|} y$, logo $\|\bar{y}\|_{c} \leq\|x\|_{X}$. Suponhamos $T((\bar{y}, 0))=\left(y_{2}, x_{2}\right)$, daí para todo $\theta \in \mathbb{K},|\theta|=1$, temos:

$$
\left\|x_{1}\right\|_{X}=\|x\|_{X}=\|(\theta \bar{y}, x)\|_{\infty}=\|T(\theta \bar{y}, x)\|_{\infty} \geq\left\|\theta x_{2}+x_{1}\right\| .
$$

Como $X$ é estritamente convexo e $x_{1} \neq 0$, pelo corolário 2 segue que $x_{2}=0$. Daí $T(\bar{y}, 0)=$ $\left(y_{2}, 0\right) \in c$ e, por consequência, $T(y, 0) \in c$. Pela generalidade no raciocínio acima, provamos (1). 
Agora, provemos que se $x \in X$ e $T((0, x))=\left(y_{1}, x_{1}\right)$, então $y_{1} \in c_{0}$. Suponhamos por absurdo que existe $x \in X$ de maneira que $T(0, x)=\left(\left(a_{n}\right)_{n}, x_{1}\right)$ e $\lim a_{n} \neq 0$. Consideremos $A=\left\{k \in \mathbb{N}: a_{k}=0\right\}$. Certamente $A$ é um conjunto finito.

Analogamente a construção de $z$ na primeira parte, podemos construir $y \in c \backslash\{0\}$ de modo que $T((y, 0))=\left(\left(b_{n}\right), x_{4}\right)$ e $b_{k}=0$ para todo $k \in A$. Além disso, observemos que por $(1)$ devemos ter $x_{4}=0$. Podemos ainda supor, sem perder generalidade, que $\|y\|_{c} \geq\|x\|_{X}$. Se isso não for verdade, basta substituir $y$ por $\alpha y$, para $\alpha=\frac{2\|x\|}{\|y\|}$. Daí temos dois possíveis casos para estudar:

Primeiro Caso. Suponhamos que $\|y\|_{c}=\left|b_{k_{0}}\right|$ para algum $k_{0} \in \mathbb{N}$.

Obviamente $k_{0} \notin A$, pois $y \neq 0$. Neste caso, vale para todo $\theta \in \mathbb{K},|\theta|=1$ :

$$
\left|b_{k_{0}}\right|=\|y\|_{c}=\|(y, \theta x)\|_{\infty}=\|T(y, \theta x)\|_{\infty} \geq\left|b_{k_{0}}+\theta a_{k_{0}}\right| .
$$

Uma contradição com o corolário 2 , pois $b_{k_{0}} \neq 0, a_{k_{0}} \neq 0$, e $\mathbb{K}$ é estritamente convexo.

Segundo Caso. Suponhamos que $\|y\|_{c}=\lim _{k \rightarrow \infty}\left|b_{k}\right|$.

Para cada $k \in \mathbb{N}$, seja $\theta_{k} \in \mathbb{K},\left|\theta_{k}\right|=1$ um escalar que satisfaz $\left|b_{k}+\theta_{k} a_{k}\right|=\left|b_{k}\right|+\left|a_{k}\right|$. Daí para cada $k \in \mathbb{N}$, temos:

$$
\|y\|_{c}=\left\|\left(y, \theta_{k} x\right)\right\|_{\infty}=\left\|T\left(y, \theta_{k} x\right)\right\|_{\infty} \geq\left|b_{k}+\theta_{k} a_{k}\right|=\left|b_{k}\right|+\left|a_{k}\right| .
$$

Segue que $\left|a_{k}\right| \leq\|y\|_{c}-\left|b_{k}\right|$ para todo $k \in \mathbb{N}$. Daí por hipótese, $\|y\|_{c}=\lim _{k \rightarrow \infty}\left|b_{k}\right|$, então $\lim _{k \rightarrow \infty}\left|a_{k}\right|=0$, uma contradição, já que estavamos supondo $\lim a_{n} \neq 0$.

Em ambos os casos, chegamos a um contradição. Isso se deve ao fato de admitirmos que $\lim a_{n} \neq 0$.

Na primeira parte provamos que se $x \in X$ e $T(0, x)=\left(y_{1}, x_{1}\right)$, com $y_{1} \in c_{0}$, então $\left\|x_{1}\right\|_{X}=$ $\|x\|_{X}$. Por fim mostramos que, se $x \in X$ e $T(0, x)=\left(y_{1}, x_{1}\right)$, então $y_{1} \in c_{0}$. Com isso, temos que se $x \in X$ e $T(0, x)=\left(y_{1}, x_{1}\right)$, então $\left\|x_{1}\right\|_{X}=\|x\|_{X}$, donde concluímos que $Q \circ T_{\left.\right|_{X}}$ é uma isometria e isso corresponde a (2). Por fim, consideremos $(y, x) \in c \oplus X$, temos:

$$
Q \circ T((y, x))=Q \circ T((y, 0))+Q \circ T((0, x))=Q \circ T((0, x))
$$

Pelo fato de $Q \circ T_{\left.\right|_{X}}$ ser uma isometria e por (1), concluímos que $T(y, x) \in c$ se, e somente se, $x=0$.

Antes de demonstrar o lema 4, vamos definir o que é o suporte de uma sequência.

Definição 13. Seja $y=\left(a_{n}\right)_{n} \in c$. Vamos considerar o seguinte subconjunto dos naturais

$$
A=\left\{k \in \mathbb{N}: a_{k} \neq 0\right\} .
$$

Chamamos o conjunto $A$ de conjunto suporte da sequência $\left(a_{n}\right)_{n}$ e o denotamos por supp $(y)$. 
Lema 4. Seja X um espaço de Banach estritamente convexo e de dimensão infinita. Seja

$$
T: c \oplus_{\infty} X \longrightarrow c \oplus_{\infty} X
$$

uma isometria cuja imagem é um subespaço de codimensão 1. Seja $P$ a projeção de $c \oplus_{\infty} X$ em c. Nessas condições, valem:

(3) Seja $D=\{k \in \mathbb{N}$ : existe $x \in X$, tal que $k \in \operatorname{supp}(P(T(0, x)))\}$. Então $D$ tem, no máximo, 1 elemento.

(4) Para todo $k \in \mathbb{N}, D \cup \operatorname{supp}\left(T\left(e_{k}, 0\right)\right)$ tem, no máximo, dois elementos.

Demonstração. Para $y=\left(a_{n}\right) \in c$, vamos definir:

$$
m(y)=\left\{k \in \mathbb{N}:\left|a_{k}\right|=\|y\|_{c}\right\} .
$$

Do lema $3(1)$, sabemos que $T(y, 0) \in c$, daí $T(y, 0)=\left(\left(b_{n}\right)_{n}, 0\right)$, para $\left(b_{n}\right)_{n} \in c$. Nessa demonstração usaremos o lema 3 (1) inúmeras vezes. Além disso, vamos cometer abusos de linguagem daqui para frente e denotar o conjunto $\left\{k \in \mathbb{N}:\left|b_{k}\right|=\left\|\left(b_{n}\right)\right\|_{c}\right\}$ por $m(T(y))$. Vamos mostrar que se $y \in c, y \neq 0$, valem:

a) $D \cap m(T(y))=\emptyset$;

b) Se $k \notin \operatorname{supp}(y)$, então $\operatorname{supp}\left(T\left(e_{k}, 0\right)\right) \cap m(T(y))=\emptyset$;

c) Se $k \notin \operatorname{supp}(y)$, então $m(T(y)) \cap\left(D \cup \operatorname{supp}\left(T\left(e_{k}, 0\right)\right)\right)=\emptyset$.

Para concluir a), consideremos $y \in c, y \neq 0$ e $T(y, 0)=\left(y^{\prime}, 0\right)$ com $y^{\prime}=\left(\delta_{n}\right)_{n}$. Vamos supor, por absurdo, $k_{0} \in D \cap m(T(y))$. Daí $\left|\delta_{k_{0}}\right|=\left\|y^{\prime}\right\|_{c}$ e existe $x \in X$ tal que $T(0, x)=\left(\left(\gamma_{n}\right), x_{5}\right)$ com $\gamma_{k_{0}} \neq 0$. Podemos supor, sem perder generalidade que $\|x\|_{X} \leq\|y\|_{c}$. Veja que se isso não for verdade basta trocar $x$ por $\alpha x \operatorname{com} \alpha=\frac{\|y\|}{2\|x\|}$. Assim, para todo $\theta \in \mathbb{K},|\theta|=1$, temos:

$$
\left|\delta_{k_{0}}\right|=\left\|y^{\prime}\right\|_{c}=\|y\|_{c}=\|(y, \theta x)\|_{\infty}=\|T(y, \theta x)\|_{\infty} \geq\left|\delta_{k_{0}}+\theta \gamma_{k_{0}}\right| .
$$

Uma contradição com o corolário 2 , pois $\delta_{k_{0}} \neq 0, \gamma_{k_{0}} \neq 0$ e $\mathbb{K}$ é estritamente convexo.

Para provar b), suponhamos $y \in c, y \neq 0, T(y, 0)=\left(y^{\prime}, 0\right)$ com $y^{\prime}=\left(\delta_{n}\right)_{n}$ e $k \in \mathbb{N}$ fixado de maneira que $k \notin \operatorname{supp}(y)$. Vamos admitir, por absurdo, que $k_{0} \in \operatorname{supp}\left(T\left(e_{k}, 0\right)\right) \cap m(T(y))$. Podemos admitir, sem perda de generalidade, que $\|y\|_{c} \geq 1$, pois se isso não for verdade basta trocar $y$ por $\alpha y$, para $\alpha=\frac{1}{\|y\|}$. Se $T\left(e_{k}, 0\right)=\left(\left(\gamma_{n}\right), 0\right)$, daí para todo $\theta \in \mathbb{K},|\theta|=1$, vale:

$$
\left|\delta_{k_{0}}\right|=\left\|y^{\prime}\right\|_{c}=\|y\|_{c}=\left\|\theta e_{k}+y\right\|_{c}=\left\|T\left(\theta e_{k}+y, 0\right)\right\|_{\infty} \geq\left|\theta \gamma_{k_{0}}+\delta_{k_{0}}\right| .
$$

Uma contradição com o corolário 2 , pois $\delta_{k_{0}} \neq 0, \gamma_{k_{0}} \neq 0$ e $\mathbb{K}$ é estritamente convexo. Dessa forma provamos b). É evidente que c) segue de a) e b).

Agora mostremos (3). Suponhamos, por absurdo, que

$$
\{m, n\} \subseteq D,
$$


sendo $m$ e $n$ dois naturais arbitrários e distintos. Vamos mostrar que, para quaisquer escalares $\alpha, \beta \in \mathbb{K},\left(\alpha e_{m}+\beta e_{n}, 0\right) \in \operatorname{ImT}$ se, e somente se, $\alpha=\beta=0$.

De fato, se $(\alpha, \beta) \in \mathbb{K} \times \mathbb{K} \backslash\{(0,0)\}$ e $\left(\alpha e_{m}+\beta e_{n}, 0\right)=T(y, 0)$, temos:

se $|\alpha| \geq|\beta|$, então $m \in D \cap m(T(y))$;

se $|\alpha| \leq|\beta|$, então $n \in D \cap m(T(y))$.

Por a), $D \cap m(T(y))=\emptyset$, portanto $\left(\alpha e_{m}+\beta e_{n}, 0\right) \notin I m T$. Pelo que acabamos de mostrar, $\left(e_{m}, 0\right) \notin \operatorname{Im} T,\left(e_{n}, 0\right) \notin \operatorname{Im} T$ e $\left(e_{m}, 0\right) \notin \mathbb{K}\left(e_{n}, 0\right) \oplus \operatorname{ImT}$, isso contradiz o fato de $T$ ter codimensão 1. Assim provamos (3).

Agora vamos provar que para cada $k \in \mathbb{N}$, o conjunto $\operatorname{supp}\left(T\left(e_{k}, 0\right)\right)$ é finito. Na verdade mostraremos que este conjunto não pode ter 4 elementos distintos. Sem perder generalidade e usando uma notação mais simples suponhamos, por absurdo, que 1,2,3 e 4 são elementos de $\operatorname{supp}\left(T\left(e_{k}, 0\right)\right)$.

Como $T$ tem codimensão 1 , existem escalares $\alpha$ e $\beta$, não ambos nulos, tais que $\left(\alpha e_{1}+\beta e_{2}, 0\right) \in$ $I m T$. Analogamente existem escalares $\gamma$ e $\delta$, não ambos nulos, tais que $\left(\gamma e_{3}+\delta e_{4}, 0\right) \in \operatorname{ImT}$. Digamos que $\left(\alpha e_{1}+\beta e_{2}, 0\right)=T\left(y^{\prime}, 0\right),\left(\gamma e_{3}+\delta e_{4}, 0\right)=T\left(y^{\prime \prime}, 0\right)$ com $y^{\prime}, y^{\prime \prime} \in c$. Existem $\lambda_{1}, \lambda_{2}$ escalares, não ambos nulos tais que $k \notin \operatorname{supp}\left(\lambda_{1} y^{\prime}+\lambda_{2} y^{\prime \prime}\right)$. Claramente temos:

$$
T\left(\lambda_{1} y^{\prime}+\lambda_{2} y^{\prime \prime}, 0\right)=\left(\lambda_{1} \alpha e_{1}+\lambda_{1} \beta e_{2}+\lambda_{2} \gamma e_{3}+\lambda_{2} \delta e_{4}, 0\right)
$$

Assim, 1,2,3 ou 4 pertencem ao conjunto $m\left(T\left(\lambda_{1} y^{\prime}+\lambda_{2} y^{\prime \prime}\right)\right) \cap \operatorname{supp}\left(T\left(e_{k}, 0\right)\right)$, sendo que $k \notin$ $\operatorname{supp}\left(\lambda_{1} y^{\prime}+\lambda_{2} y^{\prime \prime}\right)$, o que contradiz b). Assim já sabemos que para cada $k \in \mathbb{N}$, o conjunto $D \cup \operatorname{supp}\left(T\left(e_{k}, 0\right)\right)$ é finito.

Vamos mostrar que, fixado $k \in \mathbb{N}$, se $y_{1}, y_{2} \in c$ e

$$
\operatorname{supp}\left(T\left(y_{1}, 0\right)\right) \cup \operatorname{supp}\left(T\left(y_{2}, 0\right)\right) \subseteq D \cup \operatorname{supp}\left(T\left(e_{k}, 0\right)\right),
$$

então o conjunto $\left\{y_{1}, y_{2}\right\}$ é linearmente dependente. Sendo assim, suponhamos $y_{1}, y_{2} \in c$ e que vale (2.5). Sabemos que existem escalares $\alpha, \beta \in \mathbb{K}$, não ambos nulos, tais que $k \notin$ $\operatorname{supp}\left(\alpha y_{1}+\beta y_{2}\right)$. Afirmo que, nessas condições, $\operatorname{supp}\left(T\left(\alpha y_{1}+\beta y_{2}, 0\right)\right)=\emptyset$. De fato, se isso não fosse verdade, teríamos:

$$
\emptyset \neq \operatorname{supp}\left(T\left(\alpha y_{1}+\beta y_{2}, 0\right)\right) \subseteq \operatorname{supp}\left(T\left(y_{1}, 0\right)\right) \cup \operatorname{supp}\left(T\left(y_{2}, 0\right)\right) \subseteq D \cup \operatorname{supp}\left(T\left(e_{k}, 0\right)\right) .
$$

Logo, $\operatorname{supp}\left(T\left(\alpha y_{1}+\beta y_{2}, 0\right)\right)$ tem que ser finito. Então existe $k_{0} \in m\left(T\left(\alpha y_{1}+\beta y_{2}, 0\right)\right)$, note que $k_{0} \in \operatorname{supp}\left(T\left(\alpha y_{1}+\beta y_{2}, 0\right)\right) \subseteq D \cup \operatorname{supp}\left(T\left(e_{k}, 0\right)\right)$. Isso contradiz c), pois $k \notin \operatorname{supp}\left(\alpha y_{1}+\beta y_{2}\right) \mathrm{e}$ $k_{0} \in m\left(T\left(\alpha y_{1}+\beta y_{2}, 0\right)\right) \cap\left(D \cup \operatorname{supp}\left(T\left(e_{k}, 0\right)\right)\right.$.

Portanto $\operatorname{supp}\left(T\left(\alpha y_{1}+\beta y_{2}, 0\right)\right)=\emptyset, \operatorname{logo} T\left(\alpha y_{1}+\beta y_{2}, 0\right)=(0,0)$ e, pelo fato de $T$ ser uma isometria segue que $\alpha y_{1}+\beta y_{2}=0$, para escalares $\alpha$ e $\beta$ não ambos nulos. Em outras palavras, o conjunto $\left\{y_{1}, y_{2}\right\}$ é linearmente dependente.

Por fim, provemos que $\operatorname{supp}\left(T\left(e_{k}, 0\right)\right) \cup D$ tem, no máximo, 2 elementos. Suponhamos que $k_{1}$ e $k_{2}$ sejam dois naturais distintos e pertençam a $\operatorname{supp}\left(T\left(e_{k}, 0\right)\right) \cup D$. Existem $\alpha$, $\beta \in \mathbb{K}$, escalares não ambos nulos de modo que $\left(\alpha e_{k_{1}}+\beta e_{k_{2}}, 0\right) \in \operatorname{ImT}$, pois $T$ tem codimensão 1. Daí $\left(\alpha e_{k_{1}}+\beta e_{k_{2}}, 0\right)=T\left(y^{\prime}, 0\right)$, para algum $y^{\prime} \in c$. Com isso concluímos que 
$\operatorname{supp}\left(T\left(y^{\prime}, 0\right)\right) \cup \operatorname{supp}\left(T\left(e_{k}, 0\right)\right) \subseteq D \cup \operatorname{supp}\left(T\left(e_{k}, 0\right)\right)$. Pelo que acabamos de mostrar, vem que $\left\{y^{\prime}, e_{k}\right\}$ é linearmente dependente, digamos que $e_{k}=\lambda y^{\prime}$. Segue que $T\left(e_{k}, 0\right)=\lambda\left(\alpha e_{k_{1}}+\beta e_{k_{2}}, 0\right)$ e assim $\operatorname{supp}\left(T\left(e_{k}, 0\right)\right) \subseteq\left\{k_{1}, k_{2}\right\}$. Mostramos que se $k_{1}, k_{2}$ são naturais distintos e $\left\{k_{1}, k_{2}\right\} \subseteq$ $\operatorname{supp}\left(T\left(e_{k}, 0\right)\right) \cup D$, então $\operatorname{supp}\left(T\left(e_{k}, 0\right)\right) \subseteq\left\{k_{1}, k_{2}\right\}$. Daí como $D$ tem no máximo um elemento, segue que $\operatorname{supp}\left(T\left(e_{k}, 0\right)\right) \cup D$ tem, no máximo, 2 elementos.

Através dos lemas 3 e 4 podemos compreender melhor o comportamento dos deslocamentos isométricos em $c \oplus_{\infty} X$. Além disso, estamos em condições de provar o seguinte:

Teorema 10. Seja $X$ um espaço de Banach estritamente convexo. Seja $Q$ a projeção natural de $c \oplus_{\infty} X$ em $X$ e seja $T$ um deslocamento isométrico em $c \oplus_{\infty} X$, então $Q \circ T_{\left.\right|_{X}}: X \longrightarrow X$ é isometria sobrejetora e $T_{\left.\right|_{c}}$ é um deslocamento isométrico em $c$.

Demonstração. Seja $(y, x) \in c \oplus X$ arbitrário. No fim do lema 3 provamos que

$$
T(y, x) \in c \text { se, e somente se, } x=0 .
$$

Esse fato será muito útil para demonstrar este teorema. Temos dois casos a considerar:

Primeiro caso. $T(X) \subseteq X$ (mostraremos que essa hipótese não acontece).

Vamos mostrar que $X \subseteq \operatorname{Im} T$ ou $c \subseteq \operatorname{Im} T$. Suponhamos, por absurdo, que existe $x \in X$ com $(0, x) \notin \operatorname{Im} T$ e $y \in c$ com $(y, 0) \notin \operatorname{Im} T$. Pelo fato de $T$ ter codimensão 1 , existe $\lambda \in \mathbb{K}$ tal que $(0, x)=\lambda(y, 0)+T\left(y^{\prime}, x^{\prime}\right)$ para algum $\left(y^{\prime}, x^{\prime}\right) \in c \oplus X$. Daí vale

$$
(0, x)-T\left(0, x^{\prime}\right)=\lambda(y, 0)+T\left(y^{\prime}, 0\right) .
$$

Como estamos no primeiro caso, $(0, x)-T\left(0, x^{\prime}\right) \in X$. Por $(2.6), \lambda(y, 0)+T\left(y^{\prime}, 0\right) \in c$. Daí deve valer

$$
(0, x)-T\left(0, x^{\prime}\right)=\lambda(y, 0)+T\left(y^{\prime}, 0\right)=0 .
$$

Segue que $T\left(y^{\prime}, 0\right)=-\lambda(y, 0)$. Como $(0, x) \notin \operatorname{Im} T$, devemos ter $\lambda \neq 0$. Portanto $(y, 0) \in \operatorname{Im} T$, uma contradição.

Logo $X \subseteq \operatorname{Im} T$ ou $c \subseteq \operatorname{ImT}$. Por estarmos no primeiro caso e por (2.6), devemos ter $T(X)=X$ ou $T(c)=c$. Isso contradiz o fato de $T$ ser um deslocamento isométrico, pois

$$
\bigcap_{n=1}^{\infty} \operatorname{Im} T^{n}=\{0\} .
$$

Segundo Caso. Suponhamos que $T(X) \nsubseteq X$.

Nesse caso $D \neq \emptyset$. Pelo lema $4(3)$, existe $m \in \mathbb{N}$ de modo que $D=\{m\}$. Na demonstração do lema 4 provamos que $m(T(y)) \cap D=\emptyset$, para todo $y \in c \backslash\{0\}$, daí $\left(e_{m}, 0\right) \notin \operatorname{Im} T$. Então $T(c) \neq c$.

Como $\left(e_{m}, 0\right) \notin \operatorname{Im} T$ e $T$ tem codimensão 1 , para cada $x \in X$, temos:

$$
(0, x)=\lambda\left(e_{m}, 0\right)+T\left(y^{\prime}, x^{\prime}\right)=\lambda\left(e_{m}, 0\right)+T\left(y^{\prime}, 0\right)+T\left(0, x^{\prime}\right)
$$


para algum $\lambda \in \mathbb{K}$ e para algum $\left(y^{\prime}, x^{\prime}\right) \in c \oplus X$. Daí aplicando $Q$ nas igualdades acima implica que:

$$
x=Q \circ T(0, x)=Q \circ T\left(\lambda e_{m}, 0\right)+Q \circ T\left(y^{\prime}, 0\right)+Q \circ T\left(0, x^{\prime}\right) .
$$

Por (2.6), $Q \circ T\left(\lambda e_{m}, 0\right)=0$ e $Q \circ T\left(y^{\prime}, 0\right)=0$. Daí $x=Q \circ T\left(0, x^{\prime}\right)=Q \circ T_{\left.\right|_{X}}\left(0, x^{\prime}\right)$. Pelo lema $3(2), Q \circ T_{\left.\right|_{X}}$ é isometria, então é uma isometria sobrejetora. Resta-nos apenas mostrar que $T_{\left.\right|_{c}}$ é um deslocamento isométrico em $c$. Evidentemente:

$$
T_{\left.\right|_{c}} \text { é isometria e } \bigcap_{n=1}^{\infty} \operatorname{Im}\left(T_{\left.\right|_{c}}\right)^{n}=\{0\} .
$$

Já temos que $\operatorname{Im} T_{\left.\right|_{c}} \neq c$, vejamos que $T_{\left.\right|_{c}}$ tem codimensão 1. Seja $y \in c$ de modo que $y \notin \operatorname{Im} T_{\left.\right|_{c}}$, o que é equivalente a $(y, 0) \notin \operatorname{Im} T$. Daí, como $T$ tem codimensão 1 , para todo $y^{\prime} \in c$, existem $\left(y_{0}, x_{0}\right) \in c \oplus X$ e $\lambda_{0} \in \mathbb{K}$ satisfazendo:

$$
\left(y^{\prime}, 0\right)=\lambda_{0}(y, 0)+T\left(y_{0}, x_{0}\right)
$$

Por (2.6), $x_{0}=0$, daí $y^{\prime}=\lambda_{0} y+T_{\left.\right|_{c}}\left(y_{0}\right)$, e isso conclui a prova.

Apesar de não resolver a questão 1, vamos estudar o caso complexo deste problema, o qual também pode ser encontrado em [3]. Para entendermos bem o enunciado desse teorema e as propriedades nele usadas, é fundamental recordarmos o corolário 1 e a observação 1, ambos presentes no capítulo 1.

Teorema 11. O espaço de Banach $c \oplus_{\infty} c_{0}$, sobre o corpo $\mathbb{K}=\mathbb{C}$, admite um deslocamento isométrico.

Demonstração. Consideremos um conjunto infinito e enumerável de números reais que seja independente sobre os racionais, digamos $\left\{\alpha_{1}, \alpha_{2} \ldots, \alpha_{n} \ldots\right\}$. Daí, para cada $k \in \mathbb{N}$, vamos definir o número complexo

$$
z_{k}=e^{2 \pi i \alpha_{k}}
$$

Temos, dessa maneira, uma sequência de números complexos $\left(z_{n}\right)_{n}$ satisfazendo $\left|z_{k}\right|=1$, para todo $k \in \mathbb{N}$. Para $f=\left(\left(x_{n}\right),\left(y_{n}\right)\right) \in c \oplus c_{0}$ definimos:

$$
T(f)=(\bar{x}, \bar{y}), \operatorname{com} \bar{x}=\left(\sum_{n=1}^{\infty} \frac{y_{n} z_{n}}{2^{n}}, x_{1}, x_{2}, \ldots, x_{n}, \ldots\right) \text { e } \bar{y}=\left(y_{1} z_{1}, y_{2} z_{2}, \ldots, y_{n} z_{n}, \ldots\right) .
$$

Evidentemente, $T: c \oplus c_{0} \longrightarrow c \oplus c_{0}$ está bem definido e $T$ é linear. Seja $f=(x, y) \in c \oplus c_{0}$ como acima. Vejamos que $T$ é uma isometria:

$$
\begin{aligned}
\|T(x, y)\|_{\infty} & =\|\left((\bar{x}, \bar{y}) \|_{\infty}=\right. \\
& =\sup \left(\left\{\left|x_{n}\right|: n \in \mathbb{N}\right\} \cup\left\{\left|y_{n} z_{n}\right|: n \in \mathbb{N}\right\} \cup\left\{\left|\sum_{n=1}^{\infty} \frac{y_{n} z_{n}}{2^{n}}\right|\right\}\right)= \\
& =\sup \left(\left\{\left|x_{n}\right|: n \in \mathbb{N}\right\} \cup\left\{\left|y_{n}\right|: n \in \mathbb{N}\right\} \cup\left\{\left|\sum_{n=1}^{\infty} \frac{y_{n} z_{n}}{2^{n}}\right|\right\}\right) .
\end{aligned}
$$


Para cada $n \in \mathbb{N}$, temos:

$$
\left|\sum_{k=1}^{n} \frac{y_{k} z_{k}}{2^{k}}\right| \leq \sum_{k=1}^{n} \frac{\left|y_{k} z_{k}\right|}{2^{k}}=\sum_{k=1}^{n} \frac{\left|y_{k}\right|}{2^{k}} \leq\|y\|_{\infty} \sum_{k=1}^{n} \frac{1}{2^{k}} \leq\|y\|_{\infty} .
$$

Assim,

$$
\left|\sum_{k=1}^{\infty} \frac{y_{k} z_{k}}{2^{k}}\right| \leq\|y\|_{\infty} \text { e }\|T(x, y)\|_{\infty}=\sup \left(\left\{x_{n}: n \in \mathbb{N}\right\} \cup\left\{y_{n}: n \in \mathbb{N}\right\}\right)=\|(x, y)\|_{\infty} .
$$

Então $T$ é uma isometria. Vamos supor que $f \in \bigcap_{n=1}^{\infty} \operatorname{Im}\left(T^{n}\right)$ e provar que $f=0$. Daqui para frente adotaremos a seguinte notação:

$$
\begin{aligned}
T^{-k}(f) & =T^{-k}\left(\left(x_{1}, x_{2}, \ldots, x_{n}, \ldots\right),\left(y_{1}, y_{2}, \ldots, y_{n}, \ldots\right)\right)= \\
& =\left(\left(x_{-k 1}, x_{-k 2}, \ldots, x_{-k n}, \ldots\right),\left(y_{-k 1}, y_{-k 2}, \ldots, y_{-k n}, \ldots\right)\right)
\end{aligned}
$$

para todo $k \in \mathbb{N}$. Sabemos que $T\left(T^{-1}(f)\right)=f$, ou seja,

$T\left(\left(x_{-11}, x_{-12}, \ldots, x_{-1 n}, \ldots\right),\left(y_{-11}, y_{-12}, \ldots, y_{-1 n}, \ldots\right)\right)=\left(\left(x_{1}, x_{2}, \ldots, x_{n}, \ldots\right),\left(y_{1}, y_{2}, \ldots, y_{n}, \ldots\right)\right)$

Através da definição do operador $T$, tiramos que $y_{n}=y_{-1 n} z_{n}$ para todo $n \in \mathbb{N}$. Ou ainda, $y_{-1 n}=y_{n}\left(z_{n}\right)^{-1}$, para todo $n \in \mathbb{N}$. Além disso, pelo fato de $T\left(T^{-1}(f)\right)=f$, tiramos que $x_{1}=\sum_{n=1}^{\infty} \frac{y_{-1 n} z_{n}}{2^{n}}=\sum_{n=1}^{\infty} \frac{y_{n}}{2^{n}}$ e $x_{n+1}=x_{-1 n}$, para cada $n \in \mathbb{N}$.

Vamos provar por indução (em $k$ ) que $y_{-k n}=y_{n}\left(z_{n}\right)^{-k} \forall n, k \in \mathbb{N}$. Já temos o resultado para $k=1$, vamos admiti-lo para $k$ arbitrário, daí para $k+1$ temos:

$$
\begin{aligned}
T\left(T^{-(k+1)}(f)\right) & =T\left(\left(x_{-(k+1) 1}, x_{-(k+1) 2}, \ldots, x_{-(k+1) n}, \ldots\right),\left(y_{-(k+1) 1}, y_{-(k+1) 2}, \ldots, y_{-(k+1) n}, \ldots\right)\right)= \\
=T^{-k}(f) & =\left(\left(x_{-k 1}, x_{-k 2}, \ldots, x_{-k n}, \ldots\right),\left(y_{-k 1}, y_{-k 2}, \ldots, y_{-k n}, \ldots\right)\right) .
\end{aligned}
$$

Logo $y_{-k n}=z_{n} y_{-(k+1) n}$, para todo $n \in \mathbb{N}$. Daí $y_{-(k+1) n}=y_{-k n}\left(z_{n}\right)^{-1}$ para todo $n \in \mathbb{N}$. Pela hipótese de indução, $y_{-k n}=y_{n}\left(z_{n}\right)^{-k}$ e portanto temos $y_{-(k+1) n}=y_{n}\left(z_{n}\right)^{-(k+1)}$ para todo $n \in \mathbb{N}$, então está provado por indução. Assim podemos escrever:

$$
\begin{aligned}
T^{-k}(f) & =T^{-k}\left(\left(x_{1}, x_{2}, \ldots, x_{n}, \ldots\right),\left(y_{1}, y_{2}, \ldots, y_{n}, \ldots\right)\right)= \\
& =\left(\left(x_{-k 1}, x_{-k 2}, \ldots, x_{-k n}, \ldots\right),\left(y_{1}\left(z_{1}\right)^{-k}, y_{2}\left(z_{2}\right)^{-k}, \ldots, y_{n}\left(z_{n}\right)^{-k}, \ldots\right)\right) .
\end{aligned}
$$

Nossa meta agora é escrever cada $x_{n}$ em função dos elementos dos conjuntos $\left\{y_{n}: n \in \mathbb{N}\right\}$ e $\left\{z_{n}: n \in \mathbb{N}\right\}$, notemos que isso já foi feito para $x_{1}$. Vamos mostrar que $x_{n+1}=x_{-n 1}$, para todo $n \in \mathbb{N}$. Acima mostramos que $x_{2}=x_{-11}$. Notemos que:

$$
\begin{aligned}
T\left(T^{-2}(f)\right) & =T\left(\left(x_{-21}, x_{-22}, \ldots, x_{-2 n}, \ldots\right),\left(y_{-21}, y_{-22}, \ldots, y_{-2 n}, \ldots\right)\right)= \\
=T^{-1}(f) & =\left(\left(x_{-11}, x_{-12}, \ldots, x_{-1 n}, \ldots\right),\left(y_{-11}, y_{-12}, \ldots, y_{-1 n}, \ldots\right)\right)
\end{aligned}
$$

daí $x_{-21}=x_{-12}$. Pelo que tínhamos, $x_{-12}=x_{3}$, logo temos o resultado para $n=1,2$. Vamos 
provar agora para $k \in \mathbb{N}$ arbitrário, $k>2$. Temos:

$$
\begin{aligned}
T\left(T^{-k}(f)\right) & =T\left(\left(x_{-k 1}, x_{-k 2}, \ldots, x_{-k n}, \ldots\right),\left(y_{-k 1}, y_{-k 2}, \ldots, y_{-k n}, \ldots\right)\right)= \\
=T^{-(k-1)}(f) & =\left(\left(x_{-(k-1) 1}, x_{-(k-1) 2}, \ldots, x_{-(k-1) n}, \ldots\right),\left(y_{-(k-1) 1}, y_{-(k-1) 2}, \ldots, y_{-(k-1) n}, \ldots\right)\right)
\end{aligned}
$$

daí $x_{-k 1}=x_{-(k-1) 2}$. Se notarmos que $T\left(T^{-(k-1)}(f)\right)=T^{-(k-2)}(f)$ e usarmos novamente a definição do operador $T$, obtemos $x_{-(k-1) 2}=x_{-(k-2) 3}$. Daí usando que $T\left(T^{-(k-2)}(f)\right)=$ $T^{-(k-3)}(f)$, obtemos $x_{-(k-2) 3}=x_{-(k-3) 4}$ (desde que $k \geq 4$ ). Usando esse raciocínio indutivamente obtemos:

$$
x_{-k 1}=x_{-(k-1) 2}=x_{-(k-2) 3}=\ldots=x_{-2(k-1)}=x_{-1 k} .
$$

Tínhamos que $x_{-1 k}=x_{k+1}$, logo $x_{k+1}=x_{-k 1}$, é o que queríamos.

Novamente, usando a definição do operador $T$ e que $T\left(T^{-(k+1)}(f)\right)=T^{-k}(f)$, concluímos que:

$$
x_{-k 1}=\sum_{n=1}^{\infty} \frac{y_{-(k+1) n} z_{n}}{2^{n}}=\sum_{n=1}^{\infty} \frac{y_{n}\left(z_{n}\right)^{-k}}{2^{n}},
$$

sendo que na última igualdade usamos que $y_{-(k+1) n}=y_{n}\left(z_{n}\right)^{-(k+1)}$, para todo $n \in \mathbb{N}$. Portanto:

$$
x_{k+1}=\sum_{n=1}^{\infty} \frac{y_{n}\left(z_{n}\right)^{-k}}{2^{n}}
$$

para todo $k \in \mathbb{N}$. Concluímos que se $f=(x, y) \in c \oplus c_{0}$ satisfizer $f \in \bigcap_{n=1}^{\infty} \operatorname{Im}\left(T^{n}\right)$, então $x_{k+1}=\sum_{n=1}^{\infty} \frac{y_{n}\left(z_{n}\right)^{-k}}{2^{n}}$, para todo $k \in \mathbb{N}$. Daí como $x \in c$, existe $\lim _{k \rightarrow \infty} \sum_{n=1}^{\infty} \frac{y_{n}\left(z_{n}\right)^{-k}}{2^{n}}$. Suponhamos $M=\lim _{k \rightarrow \infty} \sum_{n=1}^{\infty} \frac{y_{n}\left(z_{n}\right)^{-k}}{2^{n}}$ e $L=\sum_{n=1}^{\infty} \frac{\left|y_{n}\right|}{2^{n}}$. Vamos mostrar que $M=L$ e $M=-L$, e pela unicidade do limite, concluir que $L$ deve ser 0 .

Seja $\varepsilon>0$ arbitrário, sabemos que existe $n_{0} \in \mathbb{N}$ tal que:

$$
\sum_{n=n_{0}+1}^{\infty} \frac{\left|y_{n}\right|}{2^{n}}<\varepsilon \mathrm{e}\left|\sum_{n=n_{0}+1}^{\infty} \frac{y_{n}\left(z_{n}\right)^{-k}}{2^{n}}\right|<\varepsilon,
$$

para todo $k \in \mathbb{N}$. Temos ainda que existe $k^{\prime} \in \mathbb{N}$ tal que

$$
\left|M-\sum_{n=1}^{\infty} \frac{y_{n}\left(z_{n}\right)^{-k}}{2^{n}}\right|<\varepsilon, \text { para todo } k \geq k^{\prime} .
$$

Pelo corolário 1 , existe $k_{0}>k^{\prime}$ de maneira que ||$y_{n}\left|-y_{n}\left(z_{n}\right)^{-k_{0}}\right|<\varepsilon$, para todo $n \in\left\{1,2,3, \ldots, n_{0}\right\}$. Segue que:

$$
\begin{aligned}
\left|\sum_{n=1}^{n_{0}} \frac{y_{n}\left(z_{n}\right)^{-k_{0}}}{2^{n}}-\sum_{n=1}^{n_{0}} \frac{\left|y_{n}\right|}{2^{n}}\right| & \leq \sum_{n=1}^{n_{0}} \frac{\left|y_{n}\left(z_{n}\right)^{-k_{0}}-\right| y_{n}||}{2^{n}}< \\
& <\varepsilon \sum_{n=1}^{n_{0}} \frac{1}{2^{n}}<\varepsilon .
\end{aligned}
$$


Portanto:

$$
\begin{aligned}
|M-L| & \leq\left|M-\sum_{n=1}^{\infty} \frac{y_{n}\left(z_{n}\right)^{-k_{0}}}{2^{n}}\right|+\left|\sum_{n=1}^{\infty} \frac{y_{n}\left(z_{n}\right)^{-k_{0}}}{2^{n}}-\sum_{n=1}^{\infty} \frac{\left|y_{n}\right|}{2^{n}}\right|< \\
& <\varepsilon+\left|\sum_{n=1}^{\infty} \frac{y_{n}\left(z_{n}\right)^{-k_{0}}}{2^{n}}-\sum_{n=1}^{\infty} \frac{\left|y_{n}\right|}{2^{n}}\right|= \\
& =\varepsilon+\left|\sum_{n=1}^{n_{0}} \frac{y_{n}\left(z_{n}\right)^{-k_{0}}}{2^{n}}-\sum_{n=1}^{n_{0}} \frac{\left|y_{n}\right|}{2^{n}}+\sum_{n=n_{0}+1}^{\infty} \frac{y_{n}\left(z_{n}\right)^{-k_{0}}}{2^{n}}-\sum_{n=n_{0}+1}^{\infty} \frac{\left|y_{n}\right|}{2^{n}}\right| \leq \\
& \leq \varepsilon+\left|\sum_{n=1}^{n_{0}} \frac{y_{n}\left(z_{n}\right)^{-k_{0}}}{2^{n}}-\sum_{n=1}^{n_{0}} \frac{\left|y_{n}\right|}{2^{n}}\right|+\left|\sum_{n=n_{0}+1}^{\infty} \frac{y_{n}\left(z_{n}\right)^{-k_{0}}}{2^{n}}-\sum_{n=n_{0}+1}^{\infty} \frac{\left|y_{n}\right|}{2^{n}}\right|< \\
& <\varepsilon+\varepsilon+2 \varepsilon=4 \varepsilon .
\end{aligned}
$$

Como tomamos $\varepsilon>0$ arbitrário, segue que $M=L$. Analogamente, pelo corolário 1, existe $k_{1} \geq k^{\prime}$ tal que ||$y_{n}\left|+y_{n}\left(z_{n}\right)^{-k_{1}}\right|<\varepsilon$, para todo $n \in\left\{1,2,3, \ldots, n_{0}\right\}$. Assim, o mesmo raciocínio feito acima nos dá $|M+L|<4 \varepsilon$ e, dessa maneira, $M=-L$. Com isso $L=0$, como queríamos. Daí:

$$
L=\sum_{n=1}^{\infty} \frac{\left|y_{n}\right|}{2^{n}}=0
$$

Então $y_{n}=0$, para todo $n \in \mathbb{N}$. Como $x_{k+1}=\sum_{n=1}^{\infty} \frac{y_{n}\left(z_{n}\right)^{-k}}{2^{n}}$, para todo $k \in \mathbb{N}$ e $x_{1}=\sum_{n=1}^{\infty} \frac{y_{n}}{2^{n}}$, vem que $x_{k}=0$, para todo $k \in \mathbb{N}$. Assim $f=(x, y)=(0,0)$.

Para concluir o teorema, basta mostrarmos que $T$ tem codimensão 1. Acima mostramos que, se $f=\left(\left(x_{n}\right),\left(y_{n}\right)\right) \in \operatorname{Im} T$, então $x_{1}=\sum_{n=1}^{\infty} \frac{y_{n}}{2^{n}}$. Essa condição não é apenas necessária, mas também suficiente para garantir que $f \in \operatorname{Im} T$. De fato, suponhamos que $g=\left(\left(a_{n}\right),\left(b_{n}\right)\right) \in c \oplus c_{0}$ e $a_{1}=\sum_{n=1}^{\infty} \frac{b_{n}}{2^{n}}$, nesse caso temos:

$$
g=T\left(\left(a_{2}, a_{3}, \ldots, a_{n}, \ldots\right),\left(b_{1}\left(z_{1}\right)^{-1}, b_{2}\left(z_{2}\right)^{-1}, \ldots, b_{n}\left(z_{n}\right)^{-1}, \ldots\right)\right) .
$$

Com isso em mente, podemos escrever:

$$
\operatorname{Im} T=\left\{\left(\left(x_{n}\right),\left(y_{n}\right)\right) \in c \oplus c_{0}: x_{1}=\sum_{n=1}^{\infty} \frac{y_{n}}{2^{n}}\right\} .
$$

É evidente que $\operatorname{Im} T \neq c \oplus c_{0}$. Suponhamos agora que $(x, y)=\left(\left(x_{n}\right),\left(y_{n}\right)\right) \in c \oplus c_{0}$ e $(x, y) \notin \operatorname{ImT}$. Dado $(a, b)=\left(\left(a_{n}\right),\left(b_{n}\right)\right) \in c \oplus c_{0}$, tomemos

$$
\lambda=\frac{a_{1}-\sum_{n=1}^{\infty} \frac{b_{n}}{2^{n}}}{x_{1}-\sum_{n=1}^{\infty} \frac{y_{n}}{2^{n}}} \in \mathbb{C} .
$$

Um simples cálculo mostra que $(a, b)-\lambda(x, y) \in \operatorname{Im} T$. Segue que $\operatorname{Im} T \oplus[(x, y)]=c \oplus c_{0}$. Mostramos que $T$ tem codimensão 1 e portanto é um deslocamento isométrico em $c \oplus_{\infty} c_{0}$.

Observação 4. Seja $1 \leq p<\infty$. No teorema 11, poderíamos trocar $c \oplus c_{0}$ pelo espaço de Banach $c \oplus \ell_{p}$, ainda sobre o corpo $\mathbb{K}=\mathbb{C}$. Nesse caso, definiríamos um operador linear $T_{p}$ 
exatamente como foi feito no teorema anterior, alterando apenas o conjunto em que o operador está definido. Uma prova muito parecida mostraria que $T_{p}$ é um deslocamento isométrico em $c \oplus \ell_{p}$, para $1 \leq p<\infty$. Seguindo o mesmo raciocínio, poderíamos definir um operador linear $T_{p_{0}}$ em $c_{0} \oplus \ell_{p}$ e mostrar que $T_{p_{0}}$ é um deslocamento isométrico em $c_{0} \oplus \ell_{p}$.

Com isso, enunciamos, sem demonstrar, o seguinte teorema:

Teorema 12. Seja $1 \leq p<\infty$. Os espaços de Banach $c \oplus_{\infty} \ell_{p}$ e $c_{0} \oplus_{\infty} \ell_{p}$, sobre o corpo dos complexos, admitem um deslocamento isométrico. 


\section{Capítulo 3}

\section{Deslocamentos isométricos em $E \oplus_{1} F$}

\subsection{Introdução}

Neste capítulo, estudaremos os deslocamentos isométricos definidos no espaço produto, munido com a norma $\|\cdot\|_{1}$. Neste instante, poderíamos ter a ideia de aproveitar todos os resultados do capítulo 2 , trocando a norma $\|\cdot\|_{\infty}$ pela norma $\|\cdot\|_{1}$. Isso seria um equívoco, pois nos resultados do capítulo 2, sobretudo nas desigualdades, utilizamos propriedades intrínsecas da norma $\|\cdot\|_{\infty}$. Além disso, os deslocamentos isométricos são operadores que preservam a norma, logo ao lidar com a norma $\|.\|_{1}$, precisamos de um tratamento particular.

Nosso objeto de estudo, em específico, será o produto de espaços estritamente convexos. Os teoremas 15 e 16 mostram que, se um deslocamento isométrico $T$ está definido no produto de dois espaços normados estritamente convexos e o espaço produto estiver munido com a norma $\|.\|_{1}$, então o subespaço $\operatorname{Im} T$ é retangular e $T$ é fatorável. Depois de mostrar esses teoremas, apresentaremos exemplos de espaços que não admitem deslocamentos isométricos - provaremos que esse é o caso dos espaços produto $\ell_{p} \oplus_{\infty} \ell_{q}$ e $\ell_{p} \oplus_{1} \ell_{q}$, para $1<p \neq q<\infty$.

$\mathrm{Na}$ última seção, definiremos operadores que estão relacionados com os deslocamentos isométricos, os deslocamentos reversos. Mostraremos no teorema 19 que um deslocamento reverso definido num espaço de Banach $E$ induz um deslocamento isométrico em $E^{*}$.

A menos de menção contrária, consideraremos todo espaço vetorial arbitrário de dimensão infinita e sobre um corpo $\mathbb{K}$, sendo $\mathbb{K}=\mathbb{R}$ ou $\mathbb{K}=\mathbb{C}$.

\subsection{Definições e resultados básicos}

Nesta seção provaremos alguns resultados básicos que serão usados no resto do capítulo. O foco de estudo nesse contexto são as isometrias cuja imagem tem codimensão 1, definidas no produto de dois espaços vetoriais normados estritamente convexos. Em grande parte dos resultados, a noção de ponto extremo de um conjunto convexo será fortemente usada. Vamos definir esse conceito.

Definição 14. Sejam $X$ um espaço vetorial $e A \subseteq X$ um conjunto convexo. Dizemos que $x \in A$ é um ponto extremo em (ou de) A, se para todos $u, v \in A, \lambda \in \mathbb{R} \cap] 0,1[$, a seguinte implicação for verdadeira:

$$
\text { se } x=\lambda u+(1-\lambda) v \text {, então } u=v=x .
$$


$O$ conjunto dos pontos extremos de A é denotado por ExtA.

Observação 5. Neste capítulo, quando estivermos nos referindo a um espaço vetorial normado $X$, confundiremos os conjuntos ExtX e Ext $\left(B_{X}\right)$. Assim

$$
\operatorname{Ext} X=\operatorname{Ext}\left(B_{X}\right)=\operatorname{Ext}(\{x \in X:\|x\| \leq 1\}) .
$$

Notemos que $X$ é um espaço vetorial normado, se usarmos a definição com rigor, Ext $X=\emptyset$.

Tendo em mente a definição 14 e a observação 5, os próximos dois resultados são imediatos.

Corolário 3. Seja $X$ um espaço vetorial normado arbitrário, então Ext $X \subseteq\{x \in X:\|x\|=1\}$.

Corolário 4. Seja $X$ um espaço normado estritamente convexo. Então Ext $X=\{x \in X:\|x\|=1\}$.

Além do conceito de ponto extremo, é necessário conhecer a norma $\|\cdot\|_{1}$, definida no espaço produto. Sejam $\left(E,\|\cdot\|_{E}\right)$ e $\left(F,\|\cdot\|_{F}\right)$ dois espaços de Banach. Consideremos a função

$$
\begin{aligned}
\|\cdot\|_{1}: E \oplus F & \longrightarrow \quad \mathbb{R}_{+} \\
(x, y) & \longmapsto\|x\|_{E}+\|y\|_{F}
\end{aligned}
$$

Podemos notar que $\|\cdot\|_{1}$ define uma norma em $E \oplus F$, tornando $\left(E \oplus F,\|\cdot\|_{1}\right)$ um espaço de Banach, o qual pode ser também denotado por $E \oplus_{1} F$. Nesse caso, dizemos que o espaço produto está munido com a norma $\|\cdot\|_{1}$.

Através desses conceitos, podemos notar alguns resultados básicos, esses podem ser vistos em [6]. O próximo resultado mostra que, ao munirmos o espaço produto com a norma $\|\cdot\|_{1}$, o conjunto dos pontos extremos se torna bem simples.

Proposição 15. Sejam $E$ e $F$ dois espaços vetoriais normados e seja $X=E \oplus F$ munido da norma $\|\cdot\|_{1}$, então

$$
\operatorname{Ext} X=\{(x, y) \in X: x \in \operatorname{Ext} E \text { e } y=0 \text { ou } x=0 \text { e } y \in \operatorname{Ext} F\}
$$

Demonstração. Suponhamos que $(x, y) \in \operatorname{ExtX}$ e $x \neq 0$, suponhamos por absurdo que $y \neq 0$. Como $(x, y) \in B_{X},\|x\|_{E}+\|y\|_{F} \leq 1$. Então $0<\|x\|_{E}<1$ e $0<1-\|x\|_{E}<1$. Além disso, vale

$$
\left\|\frac{y}{1-\|x\|_{E}}\right\|_{F}=\frac{\|y\|_{F}}{1-\|x\|_{E}} \leq \frac{\|y\|_{F}}{\|y\|_{F}}=1 .
$$

Assim temos

$$
(x, y)=\|x\|_{E}\left(\frac{x}{\|x\|_{E}}, 0\right)+\left(1-\|x\|_{E}\right)\left(0, \frac{y}{1-\|x\|_{E}}\right) .
$$

Uma contradição, já que estamos supondo $(x, y) \in E x t X$. Logo devemos ter $y=0$. Precisamos apenas mostrar que $x \in E x t E$, já temos que $x \in B_{E}$. Suponhamos $\lambda \in \mathbb{R}, 0<\lambda<1$ e $u, v \in B_{E}$ tais que

$$
x=\lambda u+(1-\lambda) v,
$$


daí $(x, y)=(x, 0)=\lambda(u, 0)+(1-\lambda)(v, 0)$. Como $(u, 0) \in B_{X},(v, 0) \in B_{X}$ e $(x, y) \in \operatorname{Ext} X$, segue que $(x, y)=(u, 0)=(v, 0)$. Portanto $x=u=v$. Pela generalidade, vem que $x \in$ ExtE. Portanto temos

$$
\operatorname{Ext} X \subseteq\{(x, y) \in X: x \in \operatorname{Ext} E \text { e } y=0 \text { ou } x=0 \text { e } y \in \operatorname{Ext} F\}
$$

Reciprocamente e sem perda de generalidade, tomemos $x \in \operatorname{Ext} E$, vamos provar que $(x, 0) \in$ $\operatorname{Ext} X$. Obviamente $\|(x, 0)\|_{1} \leq 1$. Suponhamos $0<\lambda<1$, um número real e $(u, z),(v, w) \in B_{X}$ tais que

$$
(x, 0)=\lambda(u, z)+(1-\lambda)(v, w) .
$$

Daí $x=\lambda u+(1-\lambda) v$. Evidentemente $u, v \in B_{E}$, como $x \in E x t E$, devemos ter

$$
x=u=v .
$$

Notemos que $\|(x, 0)\|_{1}=\|x\|_{E}=1$, pois $x \in \operatorname{ExtE}$. Daí $\|u\|_{E}=\|v\|_{E}=1$. Além disso, $(u, z),(v, w) \in B_{X}$, então $\|u\|_{E}+\|z\|_{F} \leq 1 \mathrm{e}\|v\|_{E}+\|w\|_{F} \leq 1$. Logo $z=w=0$ e tiramos que

$$
(x, 0)=(u, z)=(v, w) .
$$

Pela generalidade, segue que $(x, 0) \in \operatorname{Ext} X$, é o que queríamos. Assim vale também

$$
\operatorname{Ext} X \supseteq\{(x, y) \in X: x \in \operatorname{Ext} E \text { e } y=0 \text { ou } x=0 \text { e } y \in \operatorname{ExtF}\}
$$

então temos a tese.

Os lemas 5 e 6 serão usados na demonstração do próximo teorema, tratam-se de resultados bem simples e notáveis. Em especial, o lema 6 mostra que as imagens dos pontos extremos por uma isometria também são pontos extremos.

Lema 5. Sejam $E$ e $F$ dois espaços vetoriais normados e seja $M$ um subespaço de $\left(E \oplus F,\|\cdot\|_{1}\right)$, suponhamos $(x, 0) \in M,(0, y) \in M$ tais que $x \neq 0, y \neq 0$ e $\|x\|_{E}+\|y\|_{F}=1$. Nessas condições, $(x, y) \notin E x t M$.

Demonstração. Como $M$ é um subespaço, $(x, y) \in M$. Além disso, $\|(x, y)\|_{1}=\|x\|_{E}+\|y\|_{F}=1$. No entanto, notemos que

$$
(x, y)=\|x\|_{E}\left(\frac{x}{\|x\|_{E}}, 0\right)+\|y\|_{F}\left(0, \frac{y}{\|y\|_{F}}\right) .
$$

Evidentemente $0<\|x\|_{E}<1$ e $\|y\|_{F}=1-\|x\|_{E}$. Portanto $(x, y) \notin E x t M$.

Lema 6. Sejam $X$ e $Y$ dois espaços vetoriais normados e seja $T: X \longrightarrow Y$ uma isometria, com $M=I m T$. Então para cada $x \in X$, temos

$$
x \in E x t X \Longleftrightarrow T(x) \in \operatorname{ExtM}
$$


Demonstração. Suponhamos que $x \in \operatorname{Ext} X$ e $y=T(x)$. Suponhamos que existam $z, w \in M=$ ImT, com $\|z\| \leq 1,\|w\| \leq 1$ e $\lambda \in \mathbb{R}$, com $0<\lambda<1$ satisfazendo

$$
y=\lambda z+(1-\lambda) w .
$$

Daí existem $u, v \in X$ tais que $z=T(u)$ e $w=T(v)$. Com isso, concluímos que

$$
T(x)=y=\lambda T(u)+(1-\lambda) T(v)=T(\lambda u+(1-\lambda) v) .
$$

Como $T$ é isometria, $T$ é injetora, $\log o x=\lambda u+(1-\lambda) v$. Também, por ser $T$ uma isometria, tiramos que $\|u\| \leq 1$ e $\|v\| \leq 1$. Estamos supondo $x \in E x t X$, isso só é possível se $u=v=x$. Segue que $y=z=w$. Com isso concluímos que $y=T(x) \in E x t M$. Provamos

$$
x \in E x t X \Longrightarrow T(x) \in \operatorname{Ext} M .
$$

Para provar a recíproca, suponhamos que $y=T(x) \in \operatorname{ExtM}$, queremos provar que $x \in \operatorname{Ext} X$. Assim, suponhamos $u, v \in X$, com $\|u\| \leq 1,\|v\| \leq 1$ e $\lambda \in \mathbb{R}$, com $0<\lambda<1$ tais que

$$
x=\lambda u+(1-\lambda) v .
$$

Nesse caso, $\|T(u)\| \leq 1,\|T(v)\| \leq 1$ e temos $y=T(x)=\lambda T(u)+(1-\lambda) T(v)$. Como $y \in$ ExtM, segue que $y=T(u)=T(v)$. Mas $T$ é injetora e $T(x)=y=T(u)=T(v)$, daí

$$
x=u=v .
$$

Assim concluímos que $x \in \operatorname{Ext} X \mathrm{e}$

$$
T(x) \in E x t M \Longrightarrow x \in E x t X .
$$

Então temos a tese.

O teorema 13 pode ser visto como um análogo do lema 1, presente no capítulo 2. Mas o próximo resultado é mais fraco que esse lema.

Teorema 13. Sejam E, $F$ dois espaços normados estritamente convexos e seja $T$ uma isometria definida por $T:\left(E \oplus F,\|.\|_{1}\right) \longrightarrow\left(E \oplus F,\|.\|_{1}\right)$. Se $M=\operatorname{ImT}$ é um subespaço de codimensão 1, então para cada $x \in E, T(x, 0) \in E \times\{0\} \cup\{0\} \times F$. Uma inclusão similar a essa cabe a cada $y \in F$.

Demonstração. Como $M$ é um subespaço de codimensão 1, existe $h \in(E \oplus F)^{*}$ tal que $M=$ $\operatorname{Ker}(h)=\{(x, y) \in E \oplus F: h(x, y)=(0,0)\}$. Seja $f: E \longrightarrow \mathbb{K}$ dada por $f(x)=h(x, 0)$, para todo $x \in E$. É imediato que $f \in E^{*}$. Analogamente seja $g \in F^{*}$, dada por $g(y)=h(0, y)$, para todo $y \in F$. Logo

$$
M=\{(x, y) \in E \oplus F: f(x)+g(y)=0\} .
$$

Vamos provar o resultado para cada $x \in E$, com $\|x\|_{E}=1$. Consideremos $x \in E$ nessas condições. Como $E$ é estritamente convexo, vem que $x \in E x t E$ e, pela proposição 15 , segue que 
$(x, 0) \in \operatorname{Ext}(E \oplus F)$. Suponhamos que $T(x, 0)=\left(x_{1}, y_{1}\right)$, por ser $T$ uma isometria, resulta do lema 6 que $\left(x_{1}, y_{1}\right) \in \operatorname{ExtM}$. Para concluir a demonstração precisamos mostrar que $x_{1}=0$ ou $y_{1}=0$. Suponhamos, por absurdo, $x_{1} \neq 0$ e $y_{1} \neq 0$.

Notemos que, do fato de $\left(x_{1}, y_{1}\right) \in M$, temos $f\left(x_{1}\right)+g\left(y_{1}\right)=0$. Vejamos que $f\left(x_{1}\right) \neq 0$ e $g\left(y_{1}\right) \neq 0$. Caso contrário devemos ter $f\left(x_{1}\right)=g\left(y_{1}\right)=0$, pois $f\left(x_{1}\right)+g\left(y_{1}\right)=0$. Logo $\left(x_{1}, 0\right) \in M$ e $\left(0, y_{1}\right) \in M$. Mas $\left\|\left(x_{1}, y_{1}\right)\right\|_{1}=\|\left(T(x, 0)\left\|_{1}=\right\|(x, 0) \|_{1}=1\right.$, então $\left\|x_{1}\right\|+\left\|y_{1}\right\|=1$. Segue do lema 5 que $\left(x_{1}, y_{1}\right) \notin E x t M$, uma contradição. Portanto

$$
f\left(x_{1}\right) \neq 0 \text { e } g\left(y_{1}\right) \neq 0 .
$$

Tomemos $y \in F$ arbitrário, com $\|y\|_{F}=1$. Suponhamos que $\left(x_{2}, y_{2}\right)=T(0, y)$. Analogamente temos $\left(x_{2}, y_{2}\right) \in \operatorname{ExtM}$. Notemos que:

$$
\begin{aligned}
2 & =\|(x, y)\|_{1}=\|T(x, y)\|_{1}=\left\|\left(x_{1}+x_{2}, y_{1}+y_{2}\right)\right\|_{1}= \\
& =\left\|x_{1}+x_{2}\right\|+\left\|y_{1}+y_{2}\right\| \leq \\
& \leq\left\|x_{1}\right\|+\left\|x_{2}\right\|+\left\|y_{1}\right\|+\left\|y_{2}\right\|=2 .
\end{aligned}
$$

Então $\left\|x_{1}+x_{2}\right\|+\left\|y_{1}+y_{2}\right\|=\left\|x_{1}\right\|+\left\|x_{2}\right\|+\left\|y_{1}\right\|+\left\|y_{2}\right\|$. A desigualdade triangular nos diz que

$$
\left\|x_{1}+x_{2}\right\| \leq\left\|x_{1}\right\|+\left\|x_{2}\right\| \text { e }\left\|y_{1}+y_{2}\right\| \leq\left\|y_{1}\right\|+\left\|y_{2}\right\| .
$$

Portanto devemos ter $\left\|x_{1}+x_{2}\right\|=\left\|x_{1}\right\|+\left\|x_{2}\right\|$ e $\left\|y_{1}+y_{2}\right\|=\left\|y_{1}\right\|+\left\|y_{2}\right\|$. Assim temos dois casos para considerar:

Primeiro Caso: Suponhamos $x_{2} \neq 0$ e $y_{2} \neq 0$.

Nesse caso, como $\left(E,\|\cdot\|_{E}\right)$ e $\left(F,\|\cdot\|_{F}\right)$ são espaços estritamente convexos, pela proposição 13, existem números reais $t>0, s>0$ tais que $x_{2}=t x_{1}$ e $y_{2}=s y_{1}$. Como $\left(x_{2}, y_{2}\right) \in M$, $f\left(x_{2}\right)+g\left(y_{2}\right)=0$. Assim $t f\left(x_{1}\right)+s g\left(y_{1}\right)=0$ e temos o sistema:

$$
\left\{\begin{array}{c}
f\left(x_{1}\right)+g\left(y_{1}\right)=0 \\
t f\left(x_{1}\right)+s g\left(y_{1}\right)=0
\end{array}\right.
$$

Como $f\left(x_{1}\right) \neq 0$ e $g\left(y_{1}\right) \neq 0$, devemos ter $s=t$. Portanto $\left(x_{2}, y_{2}\right)=s\left(x_{1}, y_{1}\right)$, mas temos $\left\|\left(x_{1}, y_{1}\right)\right\|_{1}=\left\|\left(x_{2}, y_{2}\right)\right\|_{1}=1$. Segue que $s=1 \mathrm{e}$

$$
T(x, 0)=\left(x_{1}, y_{1}\right)=\left(x_{2}, y_{2}\right)=T(0, y),
$$

daí como $T$ é injetora, devemos ter $(x, 0)=(0, y)$, uma contradição, pois $x \neq 0$.

Segundo Caso: Suponhamos $x_{2}=0$ ou $y_{2}=0$ (devemos lembrar que $\left\|\left(x_{2}, y_{2}\right)\right\|_{1}=1$ ).

Sem perder generalidade suponhamos que $x_{2}=0$, então $y_{2} \neq 0$. Temos $\left\|y_{1}+y_{2}\right\|=$ $\left\|y_{1}\right\|+\left\|y_{2}\right\|$, logo pela proposição 13 exite $s \in \mathbb{R}, s>0$ tal que $y_{2}=s y_{1}$. Como $\left(0, y_{2}\right) \in M$, $f(0)+g\left(y_{2}\right)=0$, daí $g\left(s y_{1}\right)=g\left(y_{2}\right)=0$. Isso implica que $g\left(y_{1}\right)=0$, uma contradição.

Provamos por absurdo que, se $x \in E$ e $\|x\|_{E}=1$, então

$$
T(x, 0) \in E \times\{0\} \cup\{0\} \times F .
$$


Isso é suficiente para garantir a tese, tendo em mente que $T$ é linear.

Corolário 5. Sejam E, F dois espaços normados estritamente convexos e seja T uma isometria definida por $T:\left(E \oplus F,\|\cdot\|_{1}\right) \longrightarrow\left(E \oplus F,\|\cdot\|_{1}\right)$. Se $M=\operatorname{ImT}$ é um subespaço de codimensão

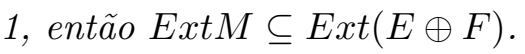

Demonstração. Fixemos $\left(x_{1}, y_{1}\right) \in$ ExtM de maneira arbitrária. Suponhamos que $\left(x_{1}, y_{1}\right)=$ $T(x, y)$. Pelo lema 6 , segue que $(x, y) \in \operatorname{Ext}(E \oplus F)$. Assim pela proposição $15, x=0 \mathrm{e}$ $y \in E x t F$ ou $y=0$ e $x \in E x t E$. Sem perder generalidade, suponhamos que $x=0$ e $y \in E x t F$. Logo $\left(x_{1}, y_{1}\right)=T(0, y)$ e pelo teorema $13, x_{1}=0$ ou $y_{1}=0$. Pelo fato de $y \in E x t F$, concluímos que $\|y\|=1$. Daí

$$
\left\|\left(x_{1}, y_{1}\right)\right\|_{1}=\|T(0, y)\|_{1}=\|(0, y)\|_{1}=1 .
$$

Se $x_{1}=0$, devemos ter $\left\|y_{1}\right\|=1$. Daí, por $F$ ser estritamente convexo, segue que $y_{1} \in E x t F$. Então, pela proposição 15 , concluímos que $\left(x_{1}, y_{1}\right) \in \operatorname{Ext}(E \oplus F)$.

Se $y_{1}=0$, então $\left\|x_{1}\right\|=1$. Daí, por $E$ ser estritamente convexo, segue que $x_{1} \in E x t E$. Então, pela proposição 15 , concluímos que $\left(x_{1}, y_{1}\right) \in \operatorname{Ext}(E \oplus F)$.

Em qualquer um dos casos temos a tese.

A seguir, o lema 7 tem um forte significado geométrico. Provaremos que se $x$ não é um ponto extremo de $E$, então existem pontos $y, z \in B_{E}$ de modo que $x$ é o ponto médio do segmento com extremos em $y$ e $z$.

Lema 7. Seja $E$ um espaço vetorial normado. Se $x \in E$ é tal que $\|x\|_{E}=1$ e $x \notin E x t E$, então existem $y \in E, z \in E$ distintos tais que $\|y\| \leq 1,\|z\| \leq 1$ e $x=\frac{1}{2} y+\frac{1}{2} z$.

Demonstração. Por hipótese $x \notin E x t E$, logo existem $u, v \in B_{E}$, distintos e $\lambda \in \mathbb{R}, 0<\lambda<1$, tais que $x=\lambda u+(1-\lambda) v$. Temos dois casos a considerar:

Primeiro Caso: $\frac{1}{2} \leq \lambda<1$.

Nesse caso, basta tomarmos $y=u$ e $z=2 x-u$. Obviamente $x=\frac{1}{2} y+\frac{1}{2} z$. Note que, se $y=z$, então $u=x=v$, uma contradição. Daí $y \neq z$. Além disso, $\|y\|=\|u\| \leq 1 \mathrm{e}$

$$
\begin{aligned}
\|z\| & =\|2 x-u\|=\|2(\lambda u+(1-\lambda) v)-u\|=\|(2 \lambda-1) u+(2-2 \lambda) v\| \leq \\
& \leq\|(2 \lambda-1) u\|+\|(2-2 \lambda) v\|=|2 \lambda-1|\|u\|+|2-2 \lambda|\|v\| \leq 1 .
\end{aligned}
$$

E assim está provado.

Segundo Caso: $0<\lambda<\frac{1}{2}$.

Nesse caso, basta tomarmos $y=v$ e $z=2 x-v$. Tudo é verificado tal como no primeiro caso.

Teorema 14. Sejam E e $F$ espaços normados estritamente convexos e $M$ um subespaço de $E \oplus_{1} F$ de codimensão 1. Suponhamos que $x \in E, y \in F$ são tais que $x \neq 0, y \neq 0,(x, 0) \notin M$, $(0, y) \notin M,(x, y) \in M e\|(x, y)\|_{1}=1$. Nessas condições, $(x, y) \in \operatorname{ExtM}$. 
Demonstração. Notemos que, por ser $M$ um subespaço de codimensão 1, existe $h \in(E \oplus F)^{*}$ tal que

$$
M=\operatorname{Ker}(h)=\{(a, b) \in E \oplus F: h(a, b)=0\} .
$$

Sejam $f \in E^{*}$ e $g \in F^{*}$ definidos por $f(a)=h(a, 0)$, para todo $a \in E$ e $g(b)=h(0, b)$, para todo $b \in F$. Nesse caso, $M$ é dado por

$$
M=\{(a, b) \in E \oplus F: f(a)+g(b)=0\} .
$$

Suponhamos, por absurdo, que $x \in E$ e $y \in F$ satisfaçam as condições do enunciado e $(x, y) \notin E x t M$. Pelo lema 7 , existem $\left(x_{1}, y_{1}\right),\left(x_{2}, y_{2}\right) \in M$ distintos, com $\left\|\left(x_{1}, y_{1}\right)\right\|_{1} \leq 1$, $\left\|\left(x_{2}, y_{2}\right)\right\|_{1} \leq 1$ tais que

$$
(x, y)=\frac{1}{2}\left(x_{1}, y_{1}\right)+\frac{1}{2}\left(x_{2}, y_{2}\right)
$$

Daqui notamos que:

$$
\begin{aligned}
2 & =\|2(x, y)\|_{1}=\left\|\left(x_{1}, y_{1}\right)+\left(x_{2}, y_{2}\right)\right\|_{1}= \\
& =\left\|x_{1}+x_{2}\right\|+\left\|y_{1}+y_{2}\right\| \leq\left\|x_{1}\right\|+\left\|x_{2}\right\|+\left\|y_{1}\right\|+\left\|y_{2}\right\|= \\
& =\left\|\left(x_{1}, y_{1}\right)\right\|_{1}+\left\|\left(x_{2}, y_{2}\right)\right\|_{1}=2 .
\end{aligned}
$$

Temos $\left\|\left(x_{1}, y_{1}\right)\right\|_{1} \leq 1,\left\|\left(x_{2}, y_{2}\right)\right\|_{1} \leq 1$ e $\left\|\left(x_{1}, y_{1}\right)\right\|_{1}+\left\|\left(x_{2}, y_{2}\right)\right\|_{1}=2$, então

$$
\left\|\left(x_{1}, y_{1}\right)\right\|_{1}=\left\|\left(x_{2}, y_{2}\right)\right\|_{1}=1
$$

Além disso, concluimos que $\left\|x_{1}+x_{2}\right\|+\left\|y_{1}+y_{2}\right\|=\left\|x_{1}\right\|+\left\|x_{2}\right\|+\left\|y_{1}\right\|+\left\|y_{2}\right\|$. Pela desigualdade triangular, temos:

$$
\left\|x_{1}+x_{2}\right\| \leq\left\|x_{1}\right\|+\left\|x_{2}\right\| \text { e }\left\|y_{1}+y_{2}\right\| \leq\left\|y_{1}\right\|+\left\|y_{2}\right\| .
$$

Portanto $\left\|x_{1}+x_{2}\right\|=\left\|x_{1}\right\|+\left\|x_{2}\right\| \mathrm{e}\left\|y_{1}+y_{2}\right\|=\left\|y_{1}\right\|+\left\|y_{2}\right\|$. Vamos estudar dois possíveis casos:

Primeiro Caso: Suponhamos $x_{i} \neq 0$ e $y_{i} \neq 0$, para todo $i=1,2$;

Nesse caso, como $E$ e $F$ são estritamente convexos, concluímos pela proposição 13 que existem números reais $s, t>0$ tais que $x_{2}=s x_{1}$ e $y_{2}=t y_{1}$. Notemos que $f\left(x_{1}\right) \neq 0$ e $g\left(y_{1}\right) \neq 0$. De fato, se $f\left(x_{1}\right)=0$, então $f\left(x_{2}\right)=s f\left(x_{1}\right)=0$. Daí $f\left(\frac{x_{1}+x_{2}}{2}\right)=\frac{1}{2} f\left(x_{1}\right)+\frac{1}{2} f\left(x_{2}\right)=0$. Como

$$
(x, y)=\left(\frac{x_{1}+x_{2}}{2}, \frac{y_{1}+y_{2}}{2}\right)
$$

concluímos por (3.1) que $(x, 0) \in M$, uma contradição. Analogamente, por $(0, y) \notin M$, concluimos que $g\left(y_{1}\right) \neq 0$. Além disso, tendo em mente (3.1) e usando que $\left(x_{1}, y_{1}\right) \in M$ e $\left(x_{2}, y_{2}\right) \in M$ chegamos ao seguinte sistema:

$$
\left\{\begin{array}{c}
f\left(x_{1}\right)+g\left(y_{1}\right)=0 \\
s f\left(x_{1}\right)+t g\left(y_{1}\right)=0
\end{array}\right.
$$


Como $f\left(x_{1}\right) \neq 0$ e $g\left(y_{1}\right) \neq 0$, devemos ter $t=s$. Logo $\left(x_{2}, y_{2}\right)=s\left(x_{1}, y_{1}\right)$. Daí como $s \in \mathbb{R}$, $s>0$ e $\left\|\left(x_{1}, y_{1}\right)\right\|_{1}=\left\|\left(x_{2}, y_{2}\right)\right\|_{1}=1$, segue que $s=1$. Assim

$$
\left(x_{1}, y_{1}\right)=\left(x_{2}, y_{2}\right)=(x, y)
$$

E assim, admitir as hipóteses do primeiro caso nos levou a uma contradição.

Segundo Caso: Suponhamos $x_{i}=0$ ou $y_{i}=0$, para algum $i \in\{1,2\}$.

Suponhamos, sem perda de generalidade, que $x_{1}=0$. Como $\left\|\left(x_{1}, y_{1}\right)\right\|_{1}=1, y_{1} \neq 0$. Como $0 \neq x=\frac{x_{1}+x_{2}}{2}, x_{2} \neq 0$. Além disso, vejamos que $y_{2} \neq 0$. Se $y_{2}=0$, então $\left(x_{2}, y_{2}\right)=\left(x_{2}, 0\right) \in M$. Daí temos $(x, 0)=\left(\frac{x_{2}}{2}, 0\right) \in M$, o que contraria a hipótese. Logo $x_{2} \neq 0$ e $y_{i} \neq 0$, para todo $i \in\{1,2\}$. Sabemos que

$$
\left\|y_{1}+y_{2}\right\|=\left\|y_{1}\right\|+\left\|y_{2}\right\| .
$$

Assim, pelo fato de $F$ ser estritamente convexo, existe $s \in \mathbb{R}, s>0$ tal que $y_{2}=s y_{1}$. Como $\left(x_{1}, y_{1}\right)=\left(0, y_{1}\right) \in M$, segue que $f(0)+g\left(y_{1}\right)=0$. Logo $g\left(y_{2}\right)=s g\left(y_{1}\right)=0$. Com isso, $g\left(y_{1}\right)=g\left(y_{2}\right)=0$. Então, por (3.1), temos

$$
(0, y)=\left(0, \frac{y_{1}+y_{2}}{2}\right) \in M
$$

Uma contradição. As contradições aconteceram por supor que $(x, y) \notin E x t M$.

\subsection{Espaços sem deslocamentos isométricos}

Nossa intenção agora é mostrar que para $1<p \neq q<\infty$, o espaço produto $\ell_{p} \oplus \ell_{q}$ não admite deslocamento isométrico. Mostraremos esse fato tanto para norma $\|\cdot\|_{1}$, quanto para a norma $\|\cdot\|_{\infty}$. Além disso, ampliaremos nosso conhecimento de como os deslocamentos isométricos se comportam no espaço produto munido com a norma $\|\cdot\|_{1}$.

Antes de começarmos, devemos definir o que são um subespaço retangular e uma isometria fatorável.

Definição 15. Sejam $E, F$ espaços vetoriais e $M \subseteq E \oplus F$ um subespaço. Dizemos que $M$ é retangular se existem subespaços $M_{1} \subseteq E$ e $M_{2} \subseteq F$, tais que $M=M_{1} \oplus M_{2}$.

Definição 16. Sejam $E, F$ espaços vetoriais normados e seja $T: E \oplus_{1} F \longrightarrow E \oplus_{1} F$ uma isometria. Dizemos que $T$ é fatorável se existem isometrias

$$
T_{1}: E \longrightarrow E \text { e } T_{2}: F \longrightarrow F
$$

tais que $T=T_{1} \times T_{2}$, isto é, $T(x, y)=\left(T_{1}(x), T_{2}(y)\right)$, para todo $(x, y) \in E \oplus F$.

Por fim, é importante introduzir uma última definição que segue a nomenclatura encontrada em [6]. Essa definição, que relaciona dois espaços de Banach, será importante para enunciarmos um teorema de uma maneira mais breve e clara.

Definição 17. Sejam E e F espaços de Banach. Dizemos que E e F são quasi isometricamente incomparáveis se forem verificadas as três seguintes condições: 
i) E e F não são isometricamente isomorfos;

ii) não existe um subespaço $M$ de $E$, com codimensão 1, isometricamente isomorfo a $F$;

iii) não existe um subespaço $N$ de $F$, com codimensão 1, isometricamente isomorfo a $E$.

Os teoremas 15 e 16 mostram que, se um deslocamento isométrico $T$ está definido no produto de dois espaços normados estritamente convexos e o espaço produto estiver munido com a norma $\|\cdot\|_{1}$, então o subespaço $\operatorname{Im} T$ é retangular e $T$ é fatorável. Na verdade esses teoremas provam algo mais forte, como veremos. Todos os resultados encontrados nesta seção podem ser vistos em [6].

Teorema 15. Sejam E e $F$ espaços normados estritamente convexos e seja $T:\left(E \oplus F,\|\cdot\|_{1}\right) \longrightarrow$ $\left(E \oplus F,\|.\|_{1}\right)$ uma isometria, com ImT $=M$. Suponhamos que $M$ tenha codimensão 1. Nessas condições, $M$ é retangular. Além disso, existe um subespaço $E_{0}$ de $E$ de codimensão 1 de modo que $M=E_{0} \oplus F$, ou existe um subespaço $F_{0}$ de $F$ de codimensão 1 de modo que $M=E \oplus F_{0}$.

Demonstração. Por hipótese, $M$ é um subespaço de $E \oplus F$ de codimensão 1. Então existem $f \in E^{*}, g \in F^{*}$ tais que

$$
M=\{(a, b) \in E \oplus F: f(a)+g(b)=0\} .
$$

Sabemos que $M \neq E \oplus F$, daí existe $x \in E$ tal que $(x, 0) \notin M$ ou existe $y \in F$ tal que $(0, y) \notin M$. Se isso não fosse verdade, como $M$ é um subespaço teríamos que $(x, y) \in M$ para todos $x \in E$ e $y \in F$, o que não acontece. Sendo assim, suponhamos sem perder generalidade que existe $x \in E$ tal que $(x, 0) \notin M$.

Afirmo que, para todo $y \in F,(0, y) \in M$. Suponhamos, por absurdo, que existe $y \in F$ tal que $(0, y) \notin M$. Temos $f(x) \neq 0$ e $g(y) \neq 0$, logo existem $t_{1}, t_{2} \in \mathbb{K}$, ambos não nulos, tais que

$$
t_{1} f(x)+t_{2} g(y)=0
$$

Portanto $\left(t_{1} x, t_{2} y\right) \in M$. Fazendo $A=\left\|\left(t_{1} x, t_{2} y\right)\right\|_{1}$, temos

$$
\left(\frac{t_{1} x}{A}, 0\right) \notin M,\left(0, \frac{t_{2} y}{A}\right) \notin M,\left\|\left(\frac{t_{1} x}{A}, \frac{t_{2} y}{A}\right)\right\|_{1}=1 \text { e }\left(\frac{t_{1} x}{A}, \frac{t_{2} y}{A}\right) \in M .
$$

Segue do teorema 14 que $\left(\frac{t_{1} x}{A}, \frac{t_{2} y}{A}\right) \in \operatorname{ExtM}$. Ainda mais, pelo corolário 5 , segue que $\left(\frac{t_{1} x}{A}, \frac{t_{2} y}{A}\right) \in$ $\operatorname{Ext}(E \oplus F)$ e pela proposição 15 devemos ter

$$
\frac{t_{1} x}{A}=0 \text { ou } \frac{t_{2} y}{A}=0
$$

Vejamos que isso é uma contradição. De fato, como $(x, 0) \notin M$ e $(0, y) \notin M$, devemos ter $x \neq 0$ e $y \neq 0$, ainda $t_{1} \neq 0$ e $t_{2} \neq 0$. Essa contradição aconteceu por admitirmos que existe $y \in F$ tal que $(0, y) \notin M$. $\operatorname{Logo}(0, y) \in M$, para todo $y \in F$. Consideremos o seguinte subespaço de $E$ :

$$
E_{0}=\{a \in E:(a, 0) \in M\} .
$$


Para concluir a tese é suficiente provar que $E_{0}$ é um subespaço de $E$ de codimensão 1 e que $M=E_{0} \oplus F$. É evidente que $E_{0}$ é um subespaço de $E$. Além disso, tomemos $\left(x_{0}, y_{0}\right) \in M$. Pelo que vimos acima, $\left(0, y_{0}\right) \in M$. Logo

$$
\left(x_{0}, 0\right)=\left(x_{0}, y_{0}\right)-\left(0, y_{0}\right) \in M
$$

Segue que $x_{0} \in E_{0}$, então $\left(x_{0}, y_{0}\right) \in E_{0} \oplus F$. Assim $M \subseteq E_{0} \oplus F$. Por outro lado, seja $\left(x_{0}, y_{0}\right) \in E_{0} \oplus F$, segue que $\left(x_{0}, 0\right) \in M$. Como $\{0\} \times F \subseteq M,\left(0, y_{0}\right) \in M$. Daí

$$
\left(x_{0}, y_{0}\right)=\left(x_{0}, 0\right)+\left(0, y_{0}\right) \in M \text {. }
$$

Concluímos que $E_{0} \oplus F \subseteq M$ e assim $M=E_{0} \oplus F$. Vejamos que $E_{0}$ tem codimensão 1 . Obviamente $E_{0}$ é um subespaço próprio, já que $x \notin E_{0}$. Tomemos $x_{1} \notin E_{0}$ arbitrário, logo $\left(x_{1}, 0\right) \notin M$ e portanto $f\left(x_{1}\right) \neq 0$. Dado $x_{2} \in E$ qualquer, para $\lambda=\frac{f\left(x_{2}\right)}{f\left(x_{1}\right)} \in \mathbb{K}$, temos

$$
f\left(x_{2}-\lambda x_{1}\right)=0,
$$

$\operatorname{assim}\left(x_{2}-\lambda x_{1}, 0\right) \in M$, ou ainda, $x_{2}-\lambda x_{1} \in E_{0}$. Provamos que $E=E_{0} \oplus\left[x_{1}\right]$, logo $E_{0}$ é um subespaço de codimensão 1 .

$\mathrm{Na}$ demonstração acima supomos que existe $x \in E$ tal que $(x, 0) \notin M$. Se ao invés disso, supuséssemos que existe $y \in F$ tal que $(0, y) \notin M$, com uma demonstração exatamente análoga concluiríamos que $M=E \oplus F_{0}$, para algum subespaço $F_{0}$ de $F$ com codimensão 1 .

Teorema 16. Sejam E e $F$ espaços de Banach estritamente convexos e de dimensão infinita. Suponhamos que E e F sejam quasi isometricamente incomparáveis. Seja $T:\left(E \oplus F,\|\cdot\|_{1}\right) \longrightarrow$ $\left(E \oplus F,\|\cdot\|_{1}\right)$ uma isometria, com ImT $=M$, suponhamos que $M$ tenha codimensão 1. Então $T$ é fatorável.

Demonstração. Pelo teorema 15 , existe um subespaço $E_{0}$ de $E$ com codimensão 1 satisfazendo $M=E_{0} \oplus F$, ou existe um subespaço $F_{0}$ de $F$ com codimensão 1 satisfazendo $M=E \oplus F_{0}$. Suponhamos, sem perder generalidade, $M=E \oplus F_{0}$, sendo $F_{0}$ exatamente como acima.

Consideremos $x \in E$, com $\|x\|_{E}=1$. Pelo fato de $T$ ser uma isometria e pelo teorema 13, concluímos que existe $x_{1} \in E$ de norma 1 tal que $T(x, 0)=\left(x_{1}, 0\right)$ ou existe $y_{1} \in F_{0}$ de norma 1 tal que $T(x, 0)=\left(0, y_{1}\right)$. Vejamos que $T(x, 0)=\left(0, y_{1}\right)$ nos leva a um absurdo. Se $T(x, 0)=\left(0, y_{1}\right)$, mostremos que $T(E \oplus\{0\}) \subseteq\{0\} \oplus F_{0}$. De fato, suponhamos que $u \in E$, $\|u\|_{E}=1$ e $T(u, 0)=\left(x_{2}, 0\right), \operatorname{logo}\left\|x_{2}\right\|_{E}=1$, já que $T$ é isometria. Temos assim

$$
\|x+u\|_{E}=\|(x+u, 0)\|_{1}=\|T(x+u, 0)\|_{1}=\left\|\left(x_{2}, y_{1}\right)\right\|_{1}=2=\|x\|_{E}+\|u\|_{E} .
$$

Pelo fato de $E$ ser estritamente convexo, segue da proposição 13 que existe $\alpha>0$ tal que $x=\alpha u$ ou $u=\alpha x$. Como $\|x\|_{E}=\|u\|_{E}=1$, devemos ter $x=u$, uma contradição pois $\left(x_{2}, 0\right) \neq$ $\left(0, y_{1}\right)$. Usando o teorema 13, provamos que se $T(x, 0)=\left(0, y_{1}\right)$, então $T(E \oplus\{0\}) \subseteq\{0\} \oplus F_{0}$. 
Suponhamos $y \in F$, com $\|y\|_{F}=1$, se $T(0, y)=\left(0, y_{2}\right)$ segue que

$$
\left\|y_{1}+y_{2}\right\|_{F}=\left\|\left(0, y_{1}+y_{2}\right)\right\|_{1}=\|T(x, y)\|_{1}=\|(x, y)\|_{1}=2 .
$$

E novamente, usando o fato de $F$ ser estritamente convexo e a proposição 13, chegamos a um absurdo. Portanto, tendo em mente o teorema 13, acabamos de provar que $T(\{0\} \oplus F) \subseteq E \oplus\{0\}$. Provamos que se $T(x, 0)=\left(0, y_{1}\right)$, então

$$
T(E \oplus\{0\}) \subseteq\{0\} \oplus F_{0} \text { e } T(\{0\} \oplus F) \subseteq E \oplus\{0\} .
$$

Consideremos $(0, y) \in\{0\} \oplus F_{0} \subseteq \operatorname{Im} T$ arbitrário, logo existe $\left(x_{0}, y_{0}\right) \in E \oplus F$ tal que $T\left(x_{0}, y_{0}\right)=(0, y)$. Por $(3.2)$, concluímos que $T\left(x_{0}, 0\right)=(0, y)$. Portanto

$$
T_{\left.\right|_{E \oplus\{0\}}}: E \oplus\{0\} \longrightarrow\{0\} \oplus F_{0}
$$

é sobrejetora e $E$ é isometricamente isomorfo a $F_{0}$. Esse último fato é uma contradição, pois $E$ e $F$ são quasi isometricamente incomparáveis. A contradição veio por admitir $T(x, 0)=\left(0, y_{1}\right)$. Portanto

$$
T(E \oplus\{0\}) \subseteq E \oplus\{0\} .
$$

Notemos que, se existe $y \in F,\|y\|_{F}=1$ e $x_{1} \in E$ tais que $T(0, y)=\left(x_{1}, 0\right)$, por analogia ao que fizemos acima, devemos ter $T(\{0\} \oplus F) \subseteq E \oplus\{0\}$. Com isso, teríamos $M=E \oplus\{0\}$, o que não acontece. Então, através do teorema 13, concluímos que

$$
T(\{0\} \oplus F) \subseteq\{0\} \oplus F_{0} .
$$

Seja $(z, w) \in \operatorname{Im} T=E \oplus F_{0}$. Assim, existe $(x, y) \in E \oplus F$ de maneira que $T(x, y)=(z, w)$. Pelo que mostramos, devemos ter

$$
T(x, 0)=(z, 0) \text { e } T(0, y)=(0, w) .
$$

Logo $T(E \oplus\{0\})=E \oplus\{0\}$ e $T(\{0\} \oplus F)=\{0\} \oplus F_{0}$. Já estamos em condições de provar que $T$ é retangular. Sejam $T_{1}: E \longrightarrow E$ e $T_{2}: F \longrightarrow F$ funções definidas como segue:

$$
T_{1}(x)=z, \text { se } T(x, 0)=(z, 0) \text { e } T_{2}(y)=w, \text { se } T(0, y)=(0, w) .
$$

Evidentemente $T_{1}$ e $T_{2}$ são isometrias lineares, $\operatorname{Im} T_{1}=E, \operatorname{Im} T_{2}=F_{0}$ e $T(x, y)=\left(T_{1}(x), T_{2}(y)\right)$, para todo $(x, y) \in E \oplus F$. Essa última propriedade pode ser denotada por $T=T_{1} \times T_{2}$. Assim, $T$ é retangular.

O teorema 17 nos traz condições para garantir que certos espaços de Banach não admitem deslocamentos isométricos. A demonstração desse resultado é simples tendo em mente os teoremas 15 e 16. No fim desta seção construiremos alguns exemplos aproveitando o próximo resultado. 
Teorema 17. Sejam E e F espaços de Banach estritamente convexos, de dimensão infinita e quasi isometricamente incomparáveis. Então $\left(E \oplus F,\|\cdot\|_{1}\right)$ não admite deslocamentos isométricos.

Demonstração. Suponhamos, por absurdo, que existe um deslocamento isométrico

$$
T:\left(E \oplus F,\|\cdot\|_{1}\right) \longrightarrow\left(E \oplus F,\|\cdot\|_{1}\right) .
$$

Pelo teorema 15, existe um subespaço $E_{0}$ de $E$ com codimensão 1 satisfazendo $\operatorname{Im} T=E_{0} \oplus F$, ou existe um subespaço $F_{0}$ de $F$ com codimensão 1 satisfazendo $\operatorname{Im} T=E \oplus F_{0}$.

Vamos supor, sem perder generalidade, que $\operatorname{Im} T=E_{0} \oplus F$. Usando o teorema 16 e adotando a mesma notação, existem isometrias $T_{1}: E \longrightarrow E$ e $T_{2}: F \longrightarrow F$ tais que $T=T_{1} \times T_{2}$, $I m T_{1}=E_{0}$ e $I m T_{2}=F$. Devemos ter em mente que a notação $T=T_{1} \times T_{2}$ significa que, para todo $(x, y) \in E \oplus F$, vale $T(x, y)=\left(T_{1}(x), T_{2}(y)\right)$. Assim, para cada $n \in \mathbb{N}$ vale

$$
T^{n}(x, y)=\left(T_{1}^{n}(x), T_{2}^{n}(y)\right), \text { para todo }(x, y) \in E \oplus F
$$

Ou ainda, para cada $n \in \mathbb{N}$, vale $T^{n}=T_{1}^{n} \times T_{2}^{n}$. Temos, pelo fato de $T_{2}$ ser sobrejetora, $F \subseteq \operatorname{Im} T_{2}^{n}$, para todo $n \in \mathbb{N}$. Logo

$$
\{0\} \oplus F \subseteq \bigcap_{n=1}^{\infty} T^{n}(E \oplus F) .
$$

Isso é uma contradição, pois $T$ é deslocamento isométrico em $E \oplus F$. Provamos a tese pela redução ao absurdo.

Notemos que, do teorema 7, decorre o seguinte teorema:

Teorema 18. Sejam E e F espaços de Banach estritamente convexos, de dimensão infinita e que não sejam isometricamente isomorfos. Então não existe deslocamento isométrico em $\left(E \oplus F,\|\cdot\|_{\infty}\right)$.

O próximo corolário é imediato desde que tenhamos um bom conhecimento dos espaços $\ell_{p}$. Na demonstração dele, assumimos que se $1<p \neq q<\infty$, então $\ell_{p}$ e $\ell_{q}$ são quasi isometricamente incomparáveis. Na referência [10], na página 75, conclui-se que esses espaços são totalmente incomparáveis.

Corolário 6. Sejam $p, q \in \mathbb{R}$ tais que $1<p \neq q<\infty$, os espaços $\ell_{p} \oplus_{1} \ell_{q}$ e $\ell_{p} \oplus_{\infty} \ell_{q}$ não admitem deslocamentos isométricos.

Demonstração. Segue dos teoremas 17 e 18, tendo em mente que $\ell_{p}$ é estritamente convexo se $1<p<\infty$ e que $\ell_{p}$ e $\ell_{q}$ são quasi isometricamente incomparáveis.

\subsection{Deslocamentos reversos em Espaços de Banach}

Nesta seção veremos operadores que estão relacionados com os deslocamentos isométricos. Os resultados abaixo, e outros, podem ser encontrados em [7]. 
Definição 18. Seja $E$ um espaço de Banach, um operador contínuo $T: E \longrightarrow E$ é um deslocamento reverso geral se satisfizer:

(1) $\operatorname{Ker}(T)$ é unidimensional;

(2) A transformação $\hat{T}: \frac{E}{K e r T} \longrightarrow E$, definida por $\hat{T}(x+K e r T)=T(x)$ é uma isometria;

(3) $\bigcup_{n=1}^{\infty} \operatorname{KerT} T^{n}$ é denso em E.

Observação 6. Daqui para frente chamaremos um deslocamento reverso geral simplesmente de deslocamento reverso.

Exemplo 5. Seja $1 \leq p<\infty$, consideremos o seguinte operador contínuo em $\ell_{p}$ :

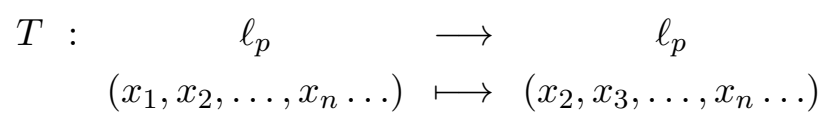

Nesse exemplo, é fácil ver que $T$ define um deslocamento reverso em $\ell_{p}$. Veremos adiante que para um espaço de Banach admitir um deslocamento reverso, ele precisa ser separável, logo $\ell_{\infty}$ não admite um deslocamento reverso.

A proposição a seguir revela a única propriedade dos deslocamentos reversos que usaremos neste trabalho. Para demonstrar esse resultado, usaremos o princípio da indução finita várias vezes.

Proposição 16. Seja E um espaço de Banach de dimensão infinita e $T: E \longrightarrow E$ um deslocamento reverso. Dai $\operatorname{Im} T=E$.

Demonstração. Consideremos $z \in E, z \neq 0$ de modo que

$$
\operatorname{Ker}(T)=[z]
$$

Vamos provar por indução que, para cada $n \in \mathbb{N}$, existem $z_{0}, z_{1}, \ldots, z_{n-1} \in E$ tais que

(a) $z_{0}=z$;

(b) $T\left(z_{i}\right)=z_{i-1}$, para cada $i=1,2, \ldots, n-1$;

(c) $\operatorname{KerT} T^{n}=\left[z_{0}, z_{1}, \ldots, z_{n-1}\right]$;

(d) $\operatorname{KerT} T^{n} \subseteq \operatorname{ImT}$.

Para $n=1$, a afirmação é $\operatorname{Ker} T=[z] \subseteq \operatorname{Im} T$. Assim, basta mostrarmos que $z \in \operatorname{Im} T$. Suponhamos, por absurdo, $z \notin \operatorname{Im} T$. Vamos mostrar, por indução, que $\operatorname{Ker} T^{k}=\operatorname{Ker} T$ para todo $k \in \mathbb{N}, k \geq 2$. Para $k=2$, dado $w \in E$ arbitrário, se $T^{2}(w)=0$, então $T(T(w))=$ $0, \operatorname{logo} T(w) \in[z]$. Mas $z \notin I m T, \log$ devemos ter $T(w)=0$ e assim $\operatorname{Ker} T^{2} \subseteq \operatorname{KerT}$. Evidentemente $\operatorname{Ker} T \subseteq K \operatorname{Ker} T^{2}$, daí mostramos o resultado para $k=2$. Vamos admitir que $\operatorname{Ker} T^{k}=\operatorname{Ker} T$ para algum $k \geq 2$. Seja $w \in E$ arbitrário, se $T^{k+1}(w)=0$, então $T\left(T^{k}(w)\right)=0$, $\operatorname{logo} T^{k}(w) \in[z]$. Novamente, pelo fato de $z \notin \operatorname{Im} T$, devemos ter $T^{k}(w)=0$. Segue que 
$K e r T^{k+1} \subseteq K e r T^{k} \subseteq \operatorname{KerT}$. Portanto $\operatorname{KerT}=\operatorname{Ker} T^{k+1}$ e está provado por indução. Veja que isso implica que $[z]=\bigcup_{n=1}^{\infty} \operatorname{KerT}^{n}$ é denso em $E$. Uma contradição pois $E$ é um espaço de dimensão infinita e $\overline{[z]}=[z]$. A contradição veio do fato de admitir que $z \notin \operatorname{Im} T$, daí devemos ter $\operatorname{Ker} T=[z] \subseteq \operatorname{Im} T$ e portanto está provado o resultado inicial para $n=1$.

Vamos assumir o resultado para $n \geq 1$ arbitrário. Sejam $z_{0}, z_{1}, \ldots, z_{n-1} \in E$, satisfazendo as quatro condições enunciadas. Como $z_{n-1} \in \operatorname{ImT}$, existe $z_{n} \in E$ de modo que $T\left(z_{n}\right)=z_{n-1}$. Vamos mostrar que $\operatorname{KerT} T^{n+1}=\left[z_{0}, z_{1}, \ldots, z_{n-1}, z_{n}\right]$, a inclusão $\left[z_{0}, z_{1}, \ldots, z_{n-1}, z_{n}\right] \subseteq \operatorname{Ker} T^{n+1}$ é imediata. Suponhamos que $w \in E$ e $T^{n+1}(w)=0$, daí $T^{n}(T(w))=0$. Como $T(w) \in \operatorname{Ker} T^{n}=$ $\left[z_{0}, z_{1}, \ldots, z_{n-1}\right]$, existem $\lambda_{0}, \lambda_{1}, \ldots, \lambda_{n-1} \in \mathbb{K}$ tais que $T(w)=\sum_{i=0}^{n-1} \lambda_{i} z_{i}=\sum_{i=0}^{n-1} \lambda_{i} T\left(z_{i+1}\right)$. Assim temos

$$
T\left(w-\sum_{i=0}^{n-1} \lambda_{i} z_{i+1}\right)=0 .
$$

Então $w-\sum_{i=0}^{n-1} \lambda_{i} z_{i+1} \in[z]$, ou ainda $w \in\left[z_{0}, z_{1}, \ldots, z_{n-1}, z_{n}\right]$, pois $z_{0}=z$. Com isso, $\operatorname{Ker} T^{n+1}=\left[z_{0}, z_{1}, \ldots, z_{n-1}, z_{n}\right]$. Para concluir o resultado para $n+1$, resta-nos mostrar que $\operatorname{Ker} T^{n+1} \subseteq \operatorname{Im} T$. Suponhamos, por absurdo, que isso não aconteça. Daí, claramente, devemos ter $z_{n} \notin \operatorname{ImT}$. Vamos mostrar, por indução, que $\operatorname{Ker} T^{n+k+1}=\operatorname{Ker} T^{n+1}$ para todo $k \in \mathbb{N}$, $k \geq 1$.

Para $k=1$, temos de mostrar que $\operatorname{Ker} T^{n+2}=\operatorname{Ker} T^{n+1}$. Seja $w \in E$ arbitrário. Se $T^{n+2}(w)=0$, então $T^{n+1}(T(w))=0$ e daí existem $\lambda_{0}, \lambda_{1}, \ldots, \lambda_{n} \in \mathbb{K}$ tais que

$$
T(w)=\sum_{i=0}^{n} \lambda_{i} z_{i}=\sum_{i=0}^{n-1} \lambda_{i} T\left(z_{i+1}\right)+\lambda_{n} z_{n} .
$$

Como $z_{n} \notin \operatorname{Im} T$, devemos ter $\lambda_{n}=0$. Daí

$$
T^{n+1}(w)=\sum_{i=0}^{n-1} \lambda_{i} T^{n+1}\left(z_{i+1}\right)=0 .
$$

Então $w \in K \operatorname{Ker} T^{n+1}$ e assim $\operatorname{Ker} T^{n+2}=K \operatorname{Ker} T^{n+1}$. Vamos admitir o resultado válido para um $k \geq 1$ arbitrário, ou seja, que $\operatorname{Ker} T^{n+k+1}=\operatorname{Ker} T^{n+1}=\left[z_{0}, z_{1}, \ldots, z_{n-1}, z_{n}\right]$. Então, para $w \in E$ qualquer, se $T^{n+k+2}(w)=0$ segue que $T^{n+k+1}(T(w))=0$. Assim, existem $\lambda_{0}, \lambda_{1}, \ldots, \lambda_{n} \in \mathbb{K}$ tais que

$$
T(w)=\sum_{i=0}^{n} \lambda_{i} z_{i}=\sum_{i=0}^{n-1} \lambda_{i} T\left(z_{i+1}\right)+\lambda_{n} z_{n} .
$$

E novamente, pelo fato de $z_{n} \notin I m T$, segue que $\lambda_{n}=0$. Com isso, $T^{n+1}(w)=0$ e podemos concluir que $\operatorname{KerT}^{n+k+2}=\operatorname{Ker} T^{n+1}$. Mostramos, pelo princípio da indução finita, que $\operatorname{Ker} T^{n+k+1}=\operatorname{Ker} T^{n+1}$, para todo $k \in \mathbb{N}$. Assim $\left[z_{0}, z_{1}, \ldots, z_{n-1}, z_{n}\right]=\operatorname{Ker} T^{n+1}=$ $\bigcup_{k=1}^{\infty} \operatorname{KerT}^{k}$ é denso em $E$, uma contradição pois $\left[z_{0}, z_{1}, \ldots, z_{n-1}, z_{n}\right]$ é fechado e $E$ tem dimensão infinita. A contradição veio do fato de admitirmos $z_{n} \notin \operatorname{Im} T$. Logo $z_{n} \in \operatorname{Im} T$ e

$$
\operatorname{KerT} T^{n+1}=\left[z_{0}, z_{1}, \ldots, z_{n-1}, z_{n}\right] \subseteq \operatorname{ImT} .
$$

Assim, provamos pelo princípio da indução finita que, para cada $n \in \mathbb{N}$, existem $z_{0}, z_{1}, \ldots, z_{n-1} \in$ 
$E$ satisfazendo as quatro condições listadas. Logo

$$
\bigcup_{n=1}^{\infty} \operatorname{Ker} T^{n} \subseteq \operatorname{Im} T
$$

Como $\hat{T}: \frac{E}{\operatorname{Ker} T} \longrightarrow E$, dada por $\hat{T}(x+\operatorname{Ker} T)=T(x)$ é uma isometria, temos que o subespaço $\operatorname{Im} T$ é fechado em $E$. Daí $E=\overline{\bigcup_{n=1}^{\infty} \operatorname{Ker} T^{n}} \subseteq \operatorname{Im} T$ e temos a tese.

Corolário 7. Seja E um espaço de Banach de dimensão infinita. Se E admite um deslocamento reverso, então E é separável.

Demonstração. Notemos que o conjunto $\bigcup_{n=1}^{\infty} K \operatorname{Ker} T^{n}$ é denso em $E$. Na demonstração da proposição 16 , provamos que $\left\{z_{k}: k=0,1, \ldots\right\}$ é um conjunto gerador de $\bigcup_{n=1}^{\infty} \operatorname{Ker} T^{n}$. Se $\mathbb{K}=\mathbb{R}$, o conjunto

$$
\left\{\sum_{j=1}^{n} q_{j} z_{j}: n \in \mathbb{N} \text { e } q_{j} \in \mathbb{Q}, \text { para todo } j \in\{1,2, \ldots, n\}\right\}
$$

é enumerável e denso em $E$. Ao passo que, para $\mathbb{K}=\mathbb{C}$, o enumerável denso é dado por

$$
\left\{\sum_{j=1}^{n}\left(a_{j}+b_{j} i\right) z_{j}: n \in \mathbb{N} \text { e } a_{j}, b_{j} \in \mathbb{Q}, \text { para todo } j \in\{1,2, \ldots, n\}\right\} \text {. }
$$

Com uma simples verificação, concluímos a demonstração.

O teorema 19 relaciona os deslocamentos isométricos com os deslocamentos reversos. Esse teorema apresenta desdobramentos quando $E$ é um espaço de Banach reflexivo.

Teorema 19. Seja E um espaço de Banach de dimensão infinita que admite um deslocamento reverso. Então $E^{*}$ admite um deslocamento isométrico.

Demonstração. Vamos construir o deslocamento isométrico e verificar as propriedades. Suponhamos $T$ um deslocamento reverso definido em $E$. Definimos $S: E^{*} \longrightarrow E^{*}$ por

$$
S\left(x^{*}\right)=x^{*} \circ T
$$

Evidentemente, $S$ está bem definida e é linear. Como $T$ é um deslocamento reverso, a definição 18 (2) nos diz que

$$
\|T(x)\|_{E}=d(x, \operatorname{Ker} T)=\inf \left\{\|x-y\|_{E}: y \in \operatorname{Ker} T\right\}, \text { para todo } x \in E \text {. }
$$

Assim, se $x^{*} \in E^{*}$ e $x \in E$, segue que

$$
\begin{aligned}
\left|S\left(x^{*}\right)(x)\right| & =\left|x^{*}(T(x))\right| \leq\left\|x^{*}\right\|_{E^{*}}\|T(x)\|_{E}=d(x, \operatorname{Ker} T)\left\|x^{*}\right\|_{E^{*}} \leq \\
& \leq\left\|x^{*}\right\|_{E^{*}}\|x-0\|_{E}=\left\|x^{*}\right\|_{E^{*}}\|x\|_{E} .
\end{aligned}
$$


Assim concluímos que $\left\|S\left(x^{*}\right)\right\|_{E^{*}} \leq\left\|x^{*}\right\|_{E^{*}}$. Por outro lado, considere $\varepsilon>0$ arbitrário. Como $\left\|x^{*}\right\|_{E^{*}}=\sup \left\{\left|x^{*}(x)\right|: x \in E,\|x\| \leq 1\right\}$, existe $x_{0} \in E$, com $\left\|x_{0}\right\| \leq 1$, satifazendo

$$
\left\|x^{*}\right\|-\left|x^{*}\left(x_{0}\right)\right| \leq \varepsilon
$$

Pela proposição 16, $T$ é sobrejetora, logo existe $y_{0} \in E$ tal que $T\left(y_{0}\right)=x_{0}$. Assim, $\left|S\left(x^{*}\right)\left(y_{0}\right)\right|=$ $\left|x^{*}\left(x_{0}\right)\right|$. Sabemos que

$$
d\left(y_{0}, \operatorname{KerT}\right)=\inf \left\{\left\|y_{0}-y\right\|_{E}: y \in \operatorname{KerT}\right\}=\left\|T\left(y_{0}\right)\right\|_{E}=\left\|x_{0}\right\|_{E} \leq 1 .
$$

Daí, existe $y_{1} \in \operatorname{Ker} T$ tal que

$$
d\left(y_{0}, \operatorname{Ker} T\right) \leq\left\|y_{0}-y_{1}\right\|_{E}<d\left(y_{0}, \operatorname{Ker} T\right)+\varepsilon \leq 1+\varepsilon
$$

Vamos supor $y_{0} \neq y_{1}$, já que se $y_{0}=y_{1} \in K e r T$ a demonstração segue trivialmente. Temos

$$
\begin{aligned}
\left|S\left(x^{*}\right)\left(\frac{y_{0}-y_{1}}{\left\|y_{0}-y_{1}\right\|}\right)\right| & =\left|x^{*}\left(\frac{T\left(y_{0}\right)-T\left(y_{1}\right)}{\left\|y_{0}-y_{1}\right\|}\right)\right|=\left|x^{*}\left(\frac{x_{0}}{\left\|y_{0}-y_{1}\right\|}\right)\right| \geq \\
& \geq \frac{\left\|x^{*}\right\|_{E^{*}}-\varepsilon}{\left\|y_{0}-y_{1}\right\|} \geq \frac{\left\|x^{*}\right\|_{E^{*}}-\varepsilon}{1+\varepsilon} .
\end{aligned}
$$

Concluímos que $\left\|S\left(x^{*}\right)\right\|_{E^{*}}=\sup \left\{\left|S\left(x^{*}\right)(x)\right|: x \in E,\|x\| \leq 1\right\} \geq \frac{\left\|x^{*}\right\|_{E^{*}-\varepsilon}}{1+\varepsilon}$ para todo $\varepsilon>0$. Portanto $\left\|S\left(x^{*}\right)\right\|_{E^{*}} \geq\left\|x^{*}\right\|_{E^{*}}$. Como tínhamos $\left\|S\left(x^{*}\right)\right\|_{E^{*}} \leq\left\|x^{*}\right\|_{E^{*}}$, segue que

$$
\left\|S\left(x^{*}\right)\right\|_{E^{*}}=\left\|x^{*}\right\|_{E^{*}}
$$

Como tomamos $x^{*} \in E^{*}$ arbitrário, concluímos que $S$ é uma isometria. Suponhamos que $\operatorname{Ker} T=[z], \operatorname{com} z \neq 0$, vamos provar que

$$
\operatorname{Im} S=\left\{x^{*} \in E^{*}: x^{*}(z)=0\right\} .
$$

É claro que $\operatorname{Im} S \subseteq\left\{x^{*} \in E^{*}: x^{*}(z)=0\right\}$. Por outro lado, suponhamos $y^{*} \in E^{*}$, tal que $y^{*}(z)=0$. Defino

$$
\begin{aligned}
& x^{*}: \quad E \quad \longrightarrow \quad \mathbb{K} \\
& x=T(u) \longmapsto y^{*}(u)
\end{aligned}
$$

Vejamos que isso define, de fato, uma função. Para todo $a, b \in E$, temos:

$$
T(a)=T(b) \Longleftrightarrow a-b \in \operatorname{Ker} T \Longleftrightarrow a-b \in[z] \Longrightarrow y^{*}(a-b)=0 \Longleftrightarrow y^{*}(a)=y^{*}(b) .
$$

Além disso, sabemos que $T$ é sobrejetora, daí $x^{*}$ é função. É fácil verificar que $x^{*}$ é linear, contínuo e $S\left(x^{*}\right)=y^{*}$. Assim provamos (3.3).

Evidentemente $\operatorname{Im} S \neq E^{*}$. Suponhamos $w^{*} \in E^{*}$ e $w^{*} \notin \operatorname{Im} S, \log w^{*}(z) \neq 0$. Fixemos $y^{*} \in E^{*}$ arbitrário. Daí, por (3.3), $y^{*}-\frac{y^{*}(z)}{w^{*}(z)} w^{*} \in \operatorname{Im} S$. Segue que $E^{*}=\left[w^{*}\right] \oplus \operatorname{Im} S$. Assim 
concluímos que $\operatorname{Im} S$ tem codimensão 1. Para concluirmos a tese, resta-nos provar que

$$
\bigcap_{n \geq 1} \operatorname{Im} S^{n}=\{0\}
$$

Para tal, fixemos $y^{*} \in \bigcap_{n \geq 1} \operatorname{Im} S^{n}$. Sejam $x \in E$ e $m \in \mathbb{N}$ arbitrários. Como $T$ é um deslocamento reverso, $\bigcup_{n \geq 1} \operatorname{Ker} T^{n}$ é denso em $E$. Segue que existem $k \in \mathbb{N}, x_{m} \in \operatorname{Ker} T^{k}$ de maneira que:

$$
\left\|x-x_{m}\right\|_{E}<\frac{1}{m}
$$

Sabemos que $y^{*} \in I m S^{k}$, portanto existe $w^{*} \in E^{*}$ tal que $y^{*}=S^{k}\left(w^{*}\right)=w^{*} \circ T^{k}$. Daí $y^{*}\left(x_{m}\right)=0$. Portanto

$$
\left|y^{*}(x)\right|=\left|y^{*}(x)-y^{*}\left(x_{m}\right)\right|=\left|y^{*}\left(x-x_{m}\right)\right| \leq\left\|y^{*}\right\|_{E^{*}}\left\|x-x_{m}\right\|_{E} \leq \frac{\left\|y^{*}\right\|_{E^{*}}}{m} .
$$

Acabamos de mostrar que, para todo $x \in E$ e para todo $m \in \mathbb{N},\left|y^{*}(x)\right| \leq \frac{\left\|y^{*}\right\|_{E^{*}}}{m}$. Desse fato podemos concluir que $y^{*}(x)=0$ para todo $x \in E$. Então $y^{*}=0$ e concluímos que $S$ é um deslocamento isométrico em $E^{*}$.

Corolário 8. Seja E um espaço de Banach reflexivo e de dimensão infinita. Se $E^{*}$ admite um deslocamento reverso, então E admite um deslocamento isométrico.

Corolário 9. Sejam $p, q \in \mathbb{R}$ tais que $1<p \neq q<\infty$, os espaços $\ell_{p} \oplus_{1} \ell_{q}$ e $\ell_{p} \oplus_{\infty} \ell_{q}$ não admitem deslocamento reverso.

Demonstração. Suponhamos, por absurdo, que $\ell_{p} \oplus_{1} \ell_{q}$ admite um deslocamento reverso. Pelo teorema $19,\left(\ell_{p} \oplus_{1} \ell_{q}\right)^{*}$ admite um deslocamento isométrico. Notemos que os espaços $\left(\ell_{p} \oplus_{1} \ell_{q}\right)^{*}$ e $\ell_{p}^{*} \oplus_{\infty} \ell_{q}^{*}$ são isometricamente isomorfos. Basta tomar a isometria linear

$$
\begin{aligned}
S: \ell_{p}^{*} \oplus_{\infty} \ell_{q}^{*} & \longrightarrow\left(\ell_{p} \oplus_{1} \ell_{q}\right)^{*} \\
(f, g) & \longmapsto f \oplus g
\end{aligned}
$$

sendo $(f \oplus g)(x, y)=f(x)+g(y)$ para todo $x \in \ell_{p}, y \in \ell_{q}$. Daí, segue que $\ell_{p}^{*} \oplus_{\infty} \ell_{q}^{*}$ admite um deslocamento isométrico. Isso contradiz o corolário 6 .

A demonstração de que $\ell_{p} \oplus_{\infty} \ell_{q}$ não admite um deslocamento reverso é análoga. Para essa, precisamos notar que os espaços $\left(\ell_{p} \oplus_{\infty} \ell_{q}\right)^{*}$ e $\ell_{p}^{*} \oplus_{1} \ell_{q}^{*}$ são isometricamente isomorfos. 


\section{Capítulo 4}

\section{Deslocamentos isométricos em $C(X)$}

\subsection{Introdução}

Seja $X$ um espaço topológico compacto e Hausdorff. Seja $\mathbb{K}$ a reta real ou o espaço dos números complexos, ambos com a topologia usual. Neste capítulo, estudaremos os deslocamentos isométricos definidos em $C(X)$, o espaço das funções contínuas definidas em $X$, com valores em $\mathbb{K}$.

Nosso primeiro passo será entender o teorema 20, esse não trata apenas de deslocamentos isométricos, mas de qualquer operador isométrico definido em $C(X)$. Através desse teorema, construiremos funções induzidas por uma isometria definida em $C(X)$, as quais possibilitam descrevê-la.

Após terminar essa primeira parte, estaremos em condições de classificar um deslocamento isométrico definido em $C(X)$ segundo dois tipos, esse é o papel do teorema 21. Passada essa etapa, estudaremos cada um dos tipos separadamente.

Essa abordagem nos possibilitará provar alguns resultados bem interessantes a respeito dos deslocamentos em $C(X)$, como é o caso do teorema 22 - demonstraremos que $C([a, b])$ não admite deslocamentos isométricos. Além disso, no corolário 11 construíremos um deslocamento isométrico num espaço topológico compacto, Hausdorff e com uma infinidade de componentes conexas. Esse último fato tem um importante significado, já que na década de 80 alguns matemáticos conjecturaram exatamente o contrário.

\subsection{Isometrias em $C(X)$}

Em todo o capítulo, consideraremos o espaço de Banach $C(X)$ munido com a norma $\|\cdot\|_{\infty}$. Essa função é definida por:

$$
\|f\|_{\infty}=\sup \{|f(x)|: x \in X\} \text {, para toda } f \in C(X) .
$$

Inicialmente, vamos considerar $C(X)$ como o espaço das funções contínuas definidas em $X$ e com valores complexos. Sejam $X$ e $Y$ dois espaços topológicos compactos e Hausdorff. Vamos admitir que

$$
T: C(X) \longrightarrow C(Y)
$$


seja uma isometria. Nossa intenção, nesta seção, é construir algumas funções induzidas por essa isometria e que serão fundamentais para o estudo dos deslocamentos isométricos em todo o capítulo. Antes de apresentar o próximo teorema, vamos introduzir algumas notações que serão nele usadas.

Sejam $x \in X$ e $y \in Y$ arbitrários, definimos:

$$
S_{x}=\{f \in C(X):\|f\|=1 \text { e }|f(x)|=1\} \text { e } R_{y}=\{g \in C(Y):\|g\|=1 \text { e }|g(y)|=1\}
$$

Ainda, para cada $x \in X$, definimos

$$
Q_{x}=\left\{y \in Y: T\left(S_{x}\right) \subseteq R_{y}\right\}
$$

Com isso, definimos

$$
Y_{0}=\bigcup_{x \in X} Q_{x}
$$

Consideraremos os espaços $C(X)$ e $C(Y)$ sobre o corpo $\mathbb{C}$ e denotamos $\Gamma=\{z \in \mathbb{C}:|z|=1\}$. Essa notação é suficiente para entender a demonstração e o enunciado do teorema 20, esse também pode ser visto em [8].

Teorema 20. Sejam $X$ e $Y$ dois espaços topológicos compactos e Hausdorff. Seja $T: C(X) \longrightarrow$ $C(Y)$ uma isometria linear. Então, o conjunto $Y_{0}$ é fechado e a relação $\phi \subseteq Y \times X$ dada por

$$
(y, x) \in \phi \text { se, e somente se, } y \in Q_{x}
$$

define uma função contínua e sobrejetora $\phi: Y_{0} \longrightarrow X$. Além disso, existe uma função $\alpha \in C(Y)$ tal que

$$
\|\alpha\|=1,|\alpha(y)|=1 \text {, para todo } y \in Y_{0}
$$

e que verifica:

$$
(T f)(y)=\alpha(y) f(\phi(y)), \text { para todos } y \in Y_{0} \text { e } f \in C(X) .
$$

Demonstração. Primeiro mostraremos que, para cada $x \in X$ e $f \in C(X)$,

$$
\text { se } f(x)=0 \text { e } y \in Q_{x} \text {, então }(T f)(y)=0 \text {. }
$$

É suficiente mostrar para $f \in C(X)$ que satisfaz $\|f\|=1$, pois $T$ é linear. Suponhamos, por absurdo, $\|f\|=1, f\left(x_{0}\right)=0$ e $(T f)\left(y_{0}\right) \neq 0$, para algum $x_{0} \in X$ e $y_{0} \in Q_{x_{0}}$. Para cada $z \in \Gamma$, consideremos

$$
g_{z}=z\left[\min \left\{1+\operatorname{Re}(\bar{z} f), \sqrt{1-(\operatorname{Im}(\bar{z} f))^{2}}\right\}+i \cdot \operatorname{Im}(\bar{z} f)\right] .
$$

É fácil ver que $g_{z}: X \longrightarrow \mathbb{C}$ é contínua, pois tanto sua parte real como sua parte imaginária são funções contínuas. É claro que $\left|g_{z}\left(x_{0}\right)\right|=|z|=1,\left\|g_{z}\right\|=1$, logo $g_{z} \in S_{x_{0}}$. Da mesma forma $\left|g_{z}\left(x_{0}\right)-f\left(x_{0}\right)\right|=1$. Notemos que

$$
\left\|g_{z}-f\right\|=|\bar{z}|\left\|g_{z}-f\right\|=\left\|\bar{z} g_{z}-\bar{z} f\right\| .
$$


Temos ainda

$$
\bar{z} g_{z}-\bar{z} f=\min \left\{1, \sqrt{1-\operatorname{Im}(\bar{z} f)^{2}}-\operatorname{Re}(\bar{z} f)\right\},
$$

daí obviamente $\left\|g_{z}-f\right\|=\left\|\bar{z} g_{z}-\bar{z} f\right\|=1$. Com isso, concluímos que $g_{z}-f \in S_{x_{0}}$. Daí, pelo fato de $y_{0} \in Q_{x_{0}}$, para cada $z \in \Gamma$, vale que:

$$
\left|\left(T g_{z}\right)\left(y_{0}\right)\right|=1 \text { e }\left|T\left(g_{z}-f\right)\left(y_{0}\right)\right|=\left|\left(T g_{z}\right)\left(y_{0}\right)-(T f)\left(y_{0}\right)\right|=1 \text {. }
$$

Com base nisso, vamos definir a seguinte função

$$
\begin{aligned}
& \kappa: \Gamma \longrightarrow \Gamma \\
& z \longmapsto\left(T g_{z}\right)\left(y_{0}\right)
\end{aligned}
$$

Vejamos que $\kappa$ é contínua. Para $z_{1}, z_{2} \in \Gamma$, temos:

$$
\begin{aligned}
\left|\kappa\left(z_{1}\right)-\kappa\left(z_{2}\right)\right| & =\left|\left(T g_{z_{1}}\right)\left(y_{0}\right)-\left(T g_{z_{2}}\right)\left(y_{0}\right)\right|= \\
& =\left|T\left(g_{z_{1}}-g_{z_{2}}\right)\left(y_{0}\right)\right| \leq \\
& \leq\left\|T\left(g_{z_{1}}-g_{z_{2}}\right)\right\|=\left\|g_{z_{1}}-g_{z_{2}}\right\|=\sup \left\{\left|g_{z_{1}}(x)-g_{z_{2}}(x)\right|: x \in X\right\} .
\end{aligned}
$$

Daí, usando que $f \in C(X)$ e $\|f\|=1$, não é difícil ver que $\kappa$ é uma função contínua. Vamos ver outras propriedades da função $\kappa$. Pela desigualdade triangular em $C(X)$, vale para cada $z \in \Gamma$ :

$$
2=\left|g_{z}\left(x_{0}\right)-g_{-z}\left(x_{0}\right)\right| \leq\left\|g_{z}-g_{-z}\right\| \leq\left\|g_{z}\right\|+\left\|g_{-z}\right\|=2
$$

Dessas desigualdades, concluímos que $\left|\left(\frac{g_{z}-g_{-z}}{2}\right)\left(x_{0}\right)\right|=1$ e $\left\|\frac{g_{z}-g_{-z}}{2}\right\|=1$. Assim, $\frac{g_{z}-g_{-z}}{2} \in S_{x_{0}}$ e, por $y_{0} \in Q_{x_{0}}$, acarreta que $\left|\left(T g_{z}\right)\left(y_{0}\right)-\left(T g_{-z}\right)\left(y_{0}\right)\right|=2=\left|\left(T g_{z}\right)\left(y_{0}\right)\right|+\left|-\left(T g_{-z}\right)\left(y_{0}\right)\right|$. Logo, por ser $\mathbb{C}$ estritamente convexo e pela proposição 13 , concluímos que

$$
\left(T g_{-z}\right)\left(y_{0}\right)=-\left(T g_{z}\right)\left(y_{0}\right), \text { para todo } z \in \Gamma \text {. }
$$

Isso é o mesmo que $\kappa(-z)=-\kappa(z)$, para todo $z \in \Gamma$. Essa propriedade nos diz que, se $z \in \kappa(\Gamma)$, então $-z \in \kappa(\Gamma)$. Notemos que $\Gamma$ é conexo e, por ser $\kappa$ contínua, o conjunto $\kappa(\Gamma) \subseteq \Gamma$ também é conexo. Daí, como $\kappa(\Gamma) \neq \emptyset$, devemos ter $\kappa(\Gamma)=\Gamma$. Segue que existe $z_{0} \in \Gamma$ tal que

$$
\left(T g_{z_{0}}\right)\left(y_{0}\right)=\kappa\left(z_{0}\right)=\frac{(T f)\left(y_{0}\right)}{\left|(T f)\left(y_{0}\right)\right|} .
$$

Pelo que tínhamos visto:

$$
\left|\left(T g_{z_{0}}\right)\left(y_{0}\right)\right|=1 \text { e }\left|\left(T g_{z_{0}}\right)\left(y_{0}\right)-(T f)\left(y_{0}\right)\right|=1 \text {. }
$$

Logo:

$$
1=\left|\left(T g_{z_{0}}\right)\left(y_{0}\right)-(T f)\left(y_{0}\right)\right|=\left|\frac{(T f)\left(y_{0}\right)}{\left|(T f)\left(y_{0}\right)\right|}-(T f)\left(y_{0}\right)\right|=1-\left|(T f)\left(y_{0}\right)\right| .
$$

Uma contradição, pois estamos supondo $(T f)\left(y_{0}\right) \neq 0$. Assim provamos (4.1) por absurdo. 
Provemos, então, a seguinte afirmação

$$
Q_{x_{1}} \cap Q_{x_{2}}=\emptyset \text {, para } x_{1}, x_{2} \in X, x_{1} \neq x_{2} .
$$

De fato, suponhamos $x_{1}, x_{2} \in X$ e $x_{1} \neq x_{2}$. Pelo lema de Urysohn, existe $f \in C(X)$ tal que $f\left(x_{1}\right)=1, f\left(x_{2}\right)=0$ e $f(x) \in[0,1] \subseteq \mathbb{R}$, para todo $x \in X$. Evidentemente, $\|f\|=1$, daí $f \in S_{x_{1}}$.

Se $y \in Q_{x_{1}}$, por definição, devemos ter $|T f(y)|=1$. Se $y \in Q_{x_{2}}$, por (4.1), segue que $|T f(y)|=0$. Assim, devemos ter $Q_{x_{1}} \cap Q_{x_{2}}=\emptyset$. Com isso, provamos (4.2).

Agora vamos provar que

$$
Q_{x} \neq \emptyset, \text { para todo } x \in X \text {. }
$$

Para tal, mostremos que a família $\left\{(T f)^{-1}(\Gamma): f \in S_{x}\right\}$ de subconjuntos fechados de $Y$ tem a propriedade da intersecção finita. Sejam $x \in X$ fixado e $f_{1}, f_{2}, \ldots, f_{n} \in S_{x}$. Definimos

$$
h=\sum_{j=1}^{n} \overline{f_{j}(x)} f_{j}
$$

Daí, $h \in C(X)$ e $|h(x)|=\left|\sum_{j=1}^{n} \overline{f_{j}(x)} f_{j}(x)\right|=\left|\sum_{j=1}^{n} 1\right|=n$. Pelo fato de $f_{j} \in S_{x}$, para cada $j \in\{1,2, \ldots, n\}$, acarreta que $\|h\| \leq \sum_{j=1}^{n}\left\|\overline{f_{j}(x)} f_{j}\right\|=n$. Assim $\|h\|=n$ e, por ser $T$ uma isometria, vem que $\|T h\|=n$. Como $Y$ é compacto, existe $y \in Y$ tal que

$$
|(T h)(y)|=\left|\sum_{j=1}^{n} \overline{f_{j}(x)}\left(T f_{j}\right)(y)\right|=n .
$$

Observemos que

$$
n=\left|\sum_{j=1}^{n} \overline{f_{j}(x)}\left(T f_{j}\right)(y)\right| \leq \sum_{j=1}^{n}\left|\overline{f_{j}(x)}\left(T f_{j}\right)(y)\right|=\sum_{j=1}^{n}\left|\left(T f_{j}\right)(y)\right| \leq n,
$$

pois $\left\|T\left(f_{j}\right)\right\|=1$, para cada $j \in\{1,2, \ldots, n\}$. Logo devemos ter $\left|\left(T f_{j}\right)(y)\right|=1$, para cada $j \in\{1,2, \ldots, n\}$. Isso é equivalente a

$$
y \in \bigcap_{j=1}^{n}\left(T f_{j}\right)^{-1}(\Gamma)
$$

Assim $\left\{(T f)^{-1}(\Gamma): f \in S_{x}\right\}$ é uma família de fechados em $Y$ com a propriedade da intersecção finita. Por ser $Y$ compacto, vem que

$$
\bigcap_{f \in S_{x}}(T f)^{-1}(\Gamma) \neq \emptyset
$$

Consideremos $y^{\prime} \in \bigcap_{f \in S_{x}}(T f)^{-1}(\Gamma)$. Se $f \in S_{x}$, então $\left|(T f)\left(y^{\prime}\right)\right|=1$, daí por definição $(T f) \in$ $R_{y^{\prime}}$ e $y^{\prime} \in Q_{x}$. Pela generalidade, provamos (4.3).

Pelo que fizemos em (4.2), a relação $\phi$ define uma função $\phi: Y_{0} \longrightarrow X$, enquanto (4.3) nos diz que $\phi$ deve ser sobrejetora. Precisamos mostrar que $\phi$ é contínua, para isso é importante 
provar a seguinte desigualdade:

$$
\text { para } f \in C(X), x_{0} \in X \text { e } x_{0}=\phi\left(y_{0}\right), \text { temos }\left|(T f)\left(y_{0}\right)\right| \geq\left|f\left(x_{0}\right)\right| .
$$

Para mostrar essa relação é suficiente provar para $f \in C(X)$ com $\|f\|=1$, já que $T$ é linear. Notemos que, se $\left|f\left(x_{0}\right)\right|=1$, como $y_{0} \in Q_{x_{0}}$, segue que $\left|T f\left(y_{0}\right)\right|=\left|f\left(x_{0}\right)\right|=1$. Se $\left|f\left(x_{0}\right)\right|=0$, por (4.1), $\left|(T f)\left(y_{0}\right)\right|=0$, daí esses dois casos estão de acordo com (4.4).

Suponhamos então $f \in C(X)$ com $\|f\|=1$. Fixemos $x_{0} \in X, x_{0}=\phi\left(y_{0}\right)$, satisfazendo $0<\left|f\left(x_{0}\right)\right|<1$. Consideremos

$$
g(x)=\frac{f(x)}{\max \left(|f(x)|,\left|f\left(x_{0}\right)\right|\right)}, \text { para todo } x \in X .
$$

Observemos que $g \in C(X)$ e $|g(x)| \leq 1$ para todo $x \in X$. Pelo fato de $\left|g\left(x_{0}\right)\right|=1$, vem que $\|g\|=1$. Como $g \in S_{x_{0}}$ e $y_{0} \in Q_{x_{0}}$, segue que $\left|(T g)\left(y_{0}\right)\right|=1$. Para cada $x \in X$, temos:

$$
|g(x)-f(x)|=\frac{|f(x)| \cdot\left|1-\max \left(|f(x)|,\left|f\left(x_{0}\right)\right|\right)\right|}{\max \left(|f(x)|,\left|f\left(x_{0}\right)\right|\right)} .
$$

Portanto $|g(x)-f(x)| \leq 1-\left|f\left(x_{0}\right)\right|$, para todo $x \in X$. É claro que $\left|g\left(x_{0}\right)-f\left(x_{0}\right)\right|=1-\left|f\left(x_{0}\right)\right|$. Com isso, concluímos que $\|g-f\|=1-\left|f\left(x_{0}\right)\right|$ e $\frac{g-f}{\|g-f\|} \in S_{x_{0}}$. Dado que $y_{0} \in Q_{x_{0}}$, devemos ter

$$
\left|T g\left(y_{0}\right)-T f\left(y_{0}\right)\right|=\|g-f\|=1-\left|f\left(x_{0}\right)\right|
$$

E daí temos

$$
1-\left|f\left(x_{0}\right)\right|=\left|T g\left(y_{0}\right)-T f\left(y_{0}\right)\right| \geq\left|T g\left(y_{0}\right)\right|-\left|T f\left(y_{0}\right)\right|=1-\left|T f\left(y_{0}\right)\right| .
$$

Dessa desigualdade concluímos (4.4). Vamos provar que, para todo $A \subseteq X$ fechado, $\phi^{-1}(A)$ é fechado em $Y$.

Seja $A \subseteq X$ um subconjunto fechado e não vazio. Evidentemente:

$$
\phi^{-1}(A)=\bigcup_{a \in A} Q_{a}
$$

Seja $y_{0} \in Y$ tal que $y_{0} \notin \phi^{-1}(A)$. Para cada $a \in A$, como $y_{0} \notin Q_{a}$, existe $f_{a} \in S_{a}$ tal que $\left|\left(T f_{a}\right)\left(y_{0}\right)\right|<1$. Portanto para cada $a \in A$, existe $f_{a} \in S_{a}$ e $\varepsilon_{a} \in \mathbb{R}, \varepsilon_{a}>0$ de maneira que $\left|\left(T f_{a}\right)\left(y_{0}\right)\right|<1-\varepsilon_{a}$.

Para cada $a \in A$, vamos considerar o seguinte subconjunto de $X$ :

$$
U_{a}=\left\{x \in X:\left|f_{a}(x)\right|>1-\varepsilon_{a}\right\}
$$

Por ser imagem inversa de aberto de $\mathbb{R}$ por função contínua, cada $U_{a}$ é aberto em $X$. Consideremos também, para cada $a \in A$, os seguintes subconjuntos de $Y$ :

$$
V_{a}=\left\{y \in Y:\left|\left(T f_{a}\right)(y)\right|>1-\varepsilon_{a}\right\} \text { e } G_{a}=\left\{y \in Y:\left|\left(T f_{a}\right)(y)\right|<1-\varepsilon_{a}\right\}
$$


Analogamente, esses conjuntos são abertos em $Y$. Notemos que, se $y \in \phi^{-1}\left(U_{a}\right)$, então $x=$ $\phi(y) \in U_{a}$. Pela definição do conjunto $U_{a}$, vem que $\left|f_{a}(x)\right|>1-\varepsilon_{a}$. Assim, usando o que provamos em (4.4), segue que $\left|\left(T f_{a}\right)(y)\right| \geq\left|f_{a}(x)\right|>1-\varepsilon_{a}$. Dessa forma $y \in V_{a}$. E com isso mostramos que, para cada $a \in A$, vale

$$
\phi^{-1}\left(U_{a}\right) \subseteq V_{a}
$$

Como o conjunto $X$ é compacto e $A \subseteq X$ é fechado, então $A$ é compacto. Além disso, para cada $a \in A$, temos $\left|f_{a}(a)\right|=1$, pois $f_{a} \in S_{a}$. Isso significa que $a \in U_{a}$ e a família

$$
\left\{U_{a}: a \in A\right\}
$$

é uma cobertura de $A$ por abertos de $X$. Logo admite uma subcobertura finita, suponhamos $a_{1}, a_{2}, \ldots, a_{n} \in A$ de modo que

$$
A \subseteq \bigcup_{j=1}^{n} U_{a_{j}}
$$

Dessa forma, temos

$$
\begin{aligned}
\bigcup_{j=1}^{n} V_{a_{j}} & \supseteq \bigcup_{j=1}^{n} \phi^{-1}\left(U_{a_{j}}\right)= \\
& =\phi^{-1}\left(\bigcup_{j=1}^{n} U_{a_{j}}\right) \supseteq \phi^{-1}(A) .
\end{aligned}
$$

Obviamente para cada $a \in A, G_{a} \cap V_{a}=\emptyset$. Logo os conjuntos $\bigcap_{j=1}^{n} G_{a_{j}}$ e $\bigcup_{j=1}^{n} V_{a_{j}}$ são disjuntos. Pelo que fizemos no início, $y_{0} \in \bigcap_{j=1}^{n} G_{a_{j}}$. Daí $\bigcap_{j=1}^{n} G_{a_{j}}$ é uma vizinhança de $y_{0}$ em $Y$ que não encontra $\phi^{-1}(A)$, pois:

$$
\phi^{-1}(A) \cap\left(\bigcap_{j=1}^{n} G_{a_{j}}\right) \subseteq\left(\bigcup_{j=1}^{n} V_{a_{j}}\right) \cap\left(\bigcap_{j=1}^{n} G_{a_{j}}\right)=\emptyset .
$$

Como tomamos $y_{0} \in Y \backslash \phi^{-1}(A)$ arbitrariamente, podemos concluir que $\phi^{-1}(A)$ é um conjunto fechado. Mostramos que se $A \subseteq X$ é fechado em $X$, então $\phi^{-1}(A)$ é fechado em $Y$. Logo $\phi$ é contínua e $Y_{0}=\phi^{-1}(X)$ é fechado em $Y$.

Mostremos a seguinte relação:

$$
f^{\prime}(x) .(T f(y))=f(x) .\left(T f^{\prime}(y)\right) \text {, para } f, f^{\prime} \in C(X), x \in X \text { e } y \in Q_{x} .
$$

Para isso, consideremos $f, f^{\prime} \in C(X), x \in X$ e $y \in Q_{x}$. Para $x$ fixado, consideremos a seguinte função:

$$
g=f^{\prime}(x) f-f(x) f^{\prime} \in C(X) .
$$

Obviamente $g(x)=0$. Daí, por (4.1) devemos ter $(T g)(y)=0$ e isso prova (4.5). Por fim, consideremos $h: X \longrightarrow \mathbb{C}$, a função que verifica $h(x)=1$ para todo $x \in X$. Seja $\alpha=T h$, como $T$ é uma isometria, devemos ter $\|\alpha\|=\|h\|=1$. Além disso, fixemos $y \in Y_{0} \subseteq Y$, seja $x=\phi(y)$. 
É evidente que $h \in S_{x}$, como $y \in Q_{x}$, devemos ter

$$
|\alpha(y)|=|(T h)(y)|=1 .
$$

Daí $|\alpha(y)|=1$, para todo $y \in Y_{0}$. Se fizermos $f^{\prime}=h$ em (4.5), concluímos que:

$$
(T f(y))=\alpha(y) . f(\phi(y)) \text { para todo } y \in Y_{0}, f \in C(X) .
$$

Assim provamos a tese.

Observação 7. Sejam $X$ e $Y$ espaços topológicos compactos e Hausdorff, vamos considerar os espaços de Banach $C(X)$ e $C(Y)$ das funções contínuas com domínio em $X$ e $Y$ (respectivamente) e contradomínio em $\mathbb{R}$. Nesse caso, uma demonstração muito parecida e mais simples que a do teorema 20 prova a tese para os espaços reais $C(X)$ e $C(Y)$.

\subsection{Classificação dos deslocamentos isométricos em $C(X)$}

Nesta seção, através do teorema 21, veremos uma maneira de classificar os deslocamentos isométricos em $C(X)$. Ao fazer isso, desenvolveremos uma noção mais forte de como esses operadores agem no espaço das funções contínuas e isso será importante para decidir, em alguns casos, se $C(X)$ admite ao menos um deslocamento isométrico. No restante do capítulo, $X$ denotará um espaço topológico compacto e Hausdorff. Consideraremos $C(X)$ como o espaço de todas as funções contínuas definidas em $X$ e com valores em $\mathbb{K}$, podendo esse ser $\mathbb{R}$ ou $\mathbb{C}$.

Com o uso do teorema 20, o próximo resultado é quase imediato. Os resultados desta seção podem ser encontrados em [9].

Teorema 21. Suponhamos que $T$ seja um deslocamento isométrico em $C(X)$. Então existe um conjunto $X_{0} \subseteq X$ fechado, uma função contínua e sobrejetora $\psi: X_{0} \longrightarrow X$ e uma função $w \in C\left(X_{0}\right)$ satisfazendo

$$
|w(x)|=1 e(T f)(x)=w(x) f(\psi(x)) \text {, para todos } x \in X_{0}, f \in C(X) .
$$

Além disso, existem duas possibilidades para o conjunto $X_{0}$ :

(i) $X_{0}=X \backslash\{p\}$, sendo $p$ um ponto isolado de $X$;

(ii) $X_{0}=X$.

Demonstração. Pelo teorema 20, existe um subconjunto $X_{0} \subseteq X$ fechado, uma aplicação contínua e sobrejetora $\psi: X_{0} \longrightarrow X$ e uma função $w \in C\left(X_{0}\right)$ tais que

$$
|w(x)|=1 \text { e }(T f)(x)=w(x) f(\psi(x)), \text { para todos } x \in X_{0}, f \in C(X) .
$$

Na demonstração do teorema 20, em (4.3), provamos que o conjunto $X_{0}$ é não vazio. Resta-nos provar que o conjunto $X_{0}$ pode ser apenas dos dois tipos descritos acima. De fato, suponhamos por absurdo que $X \backslash X_{0}$ contenha dois pontos distintos, digamos $x_{1}$ e $x_{2}$. 
Notemos que os conjuntos $X_{0} \cup\left\{x_{1}\right\}$ e $X_{0} \cup\left\{x_{2}\right\}$ são fechados. Pelo lema de Urysohn, existem funções $f_{1}: X \longrightarrow[0,1], f_{2}: X \longrightarrow[0,1]$ em $C(X)$ tais que

$$
f_{1}\left(x_{1}\right)=f_{2}\left(x_{2}\right)=1, f_{1}\left(x_{2}\right)=f_{2}\left(x_{1}\right)=0 \text { e } f_{1}(x)=f_{2}(x)=0, \text { para cada } x \in X_{0} .
$$

Vamos mostrar que nenhuma combinação linear não trivial de $f_{1}$ e $f_{2}$ pertence a $\operatorname{ImT}$. Suponhamos $g=\lambda_{1} f_{1}+\lambda_{2} f_{2} \in \operatorname{Im} T$. Assim existe $h \in C(X)$ tal que $g=T h$ e portanto temos

$$
g(x)=(T h)(x)=w(x) h(\psi(x)), \text { para todo } x \in X_{0} .
$$

Como $f_{1}$ e $f_{2}$ se anulam em $X_{0}$, segue que $g_{\left.\right|_{X_{0}}} \equiv 0$. Sabemos que $|w(x)|=1$ para todo $x \in X_{0}$, $\operatorname{logo} w(x) \neq 0$ para todo $x \in X_{0}$. Então devemos ter

$$
h(\psi(x))=0 \text {, para todo } x \in X_{0} .
$$

Pelo fato de $\psi: X_{0} \longrightarrow X$ ser sobrejetora, devemos ter $h \equiv 0$. Com isso $g=T h=0$. Daí a única combinação linear entre $f_{1}$ e $f_{2}$ que pertence a $\operatorname{Im} T$ é a trivial. Como $f_{1}$ e $f_{2}$ são linearmente independentes em $C(X)$, isso contradiz o fato de $T$ ser um operador cuja imagem tem codimensão 1. A contradição ocorreu por supormos que $X \backslash X_{0}$ tem dois elementos distintos.

Então $X_{0}=X$ ou $X_{0}=X \backslash\{p\}$. Nesse último caso $p$ é um ponto isolado, já que o conjunto $X_{0}$ é fechado.

Observação 8. Daqui para frente, $T$ sempre irá denotar um deslocamento isométrico em $C(X)$, sendo $X$ um espaço topológico compacto e Hausdorff. As letras $\psi$ e $w$ irão denotar as funções definidas no teorema 21, $X_{0}$ será sempre o domínio de $\psi$ e p será o ponto isolado de $X$ quando estivermos no caso (i) do teorema 21. Com essa convenção, a leitura se torna mais agradável e os enunciados se tornam mais simples e diretos.

Corolário 10. Seja $T: C(X) \longrightarrow C(X)$ um deslocamento isométrico. Suponhamos $f \in C(X)$ de modo que $f(x)=0$, para todo $x \in X_{0}$. Então $f \in \operatorname{ImT}$ se, e somente se, $f=0$.

Demonstração. A prova deste fato está na demonstração do teorema 21.

Usando os resultados obtidos pelo teorema 21, podemos classificar um deslocamento isométrico definido em $C(X)$ da seguinte forma:

Definição 19. Seja $T: C(X) \longrightarrow C(X)$ um deslocamento isométrico.

(I) Dizemos que $T$ é do tipo I se $X_{0}$ pode ser escolhido de modo que $X_{0} \neq X$, satisfazendo todas as condições do teorema 21;

(II) Dizemos que $T$ é do tipo II se é possivel tomar $X_{0}=X$, satisfazendo todas as condições do teorema 21.

Observação 9. Como veremos adiante, um deslocamento isométrico $T$, em $C(X)$, pode ser simultaneamente dos tipos I e II.

Vejamos alguns exemplos de deslocamentos isométricos em $C(X)$ e como classificamos esses de acordo com a definição 19. Nos três exemplos que seguem consideraremos $K=\mathbb{C}$. 
Exemplo 6. Seja $X$ o compactificado de Alexandroff dos números naturais, $X=\mathbb{N} \cup\{\infty\}$. Então $C(X)$ é isometricamente isomorfo ao espaço de todas as sequências convergentes. Ao identificarmos $C(X)$ com c, vamos definir $T$ como segue:

$$
\begin{array}{ccc}
T: C(X) & \longrightarrow c(X) \\
x=\left(x_{n}\right)_{n} & \longmapsto\left(0, x_{1}, x_{2}, \ldots\right)
\end{array}
$$

É evidente que $T$ é um deslocamento isométrico em $C(X) \approx c$. Sejam $X_{0}=X \backslash\{1\}$ e $\psi a$ função

$$
\begin{gathered}
\psi: X_{0} \longrightarrow X \\
\psi(x)=\left\{\begin{array}{rrr}
x-1, & \text { se } & x \in \mathbb{N} \\
\infty, & \text { se } & x=\infty
\end{array}\right.
\end{gathered}
$$

Vamos definir ainda, $w: X_{0} \longrightarrow \mathbb{C}$ de modo que $w(x)=1$ para todo $x \in X_{0}$. Daí é fácil verificar que $X_{0}, \psi$, w e $p=1$ estão de acordo com o teorema 21. Em especial, é rápido ver que

$$
(T f)(x)=w(x) f(\psi(x)), \text { para todos } x \in X_{0}, f \in C(X) .
$$

Portanto, pela definição 19, T é um deslocamento isométrico tipo I.

Exemplo 7. Seja $X$ como no exemplo 6, vamos identificar $C(X)$ com c. Seja $T$ o operador linear definido por

$$
\begin{array}{ccc}
T: C(X) & \longrightarrow & C(X) \\
x=\left(x_{n}\right)_{n} & \longmapsto & \left(-x_{1}, x_{1}, x_{2}, x_{3}, \ldots\right)
\end{array}
$$

Com efeito, vamos mostrar que $T$ é, de fato, um deslocamento isométrico. Suponhamos $\left(x_{n}\right)_{n} \in$ $\bigcap_{n=1}^{\infty} \operatorname{Im} T^{n}$, dai segue que $x_{k}=-x_{k+1}$ para todo $k \in \mathbb{N}$. Temos que $\left(x_{n}\right)_{n}$ é convergente, suponhamos $x_{n} \rightarrow L$, daí

$$
L=\lim _{n \rightarrow \infty} x_{n}=-\lim _{n \rightarrow \infty} x_{n+1}=-L .
$$

Portanto devemos ter $L=0$. É evidente que $\left(x_{1}, x_{2}, \ldots\right)=\left(x_{1},-x_{1}, x_{1},-x_{1}, \ldots\right)$. Assim devemos ter $\left(x_{n}\right)_{n}=0$. Acabamos de mostrar que

$$
\bigcap_{n=1}^{\infty} \operatorname{Im} T^{n}=\{0\} .
$$

Claramente $\operatorname{Im} T=\left\{\left(x_{n}\right)_{n} \in c: x_{1}=-x_{2}\right\}$. Logo $\operatorname{Im} T \neq C(X)$. Fixemos $\left(y_{n}\right)_{n} \in c \backslash \operatorname{Im} T$ arbitrário, daí $y_{1}+y_{2} \neq 0$. Dado $\left(x_{n}\right)_{n} \in c$ qualquer, para $\lambda=\frac{x_{1}+x_{2}}{y_{1}+y_{2}} \in \mathbb{C}$, temos

$$
\left(x_{n}\right)-\lambda\left(y_{n}\right) \in \operatorname{ImT}
$$

Portanto $\operatorname{Im} T \oplus\left[\left(y_{n}\right)_{n}\right]=c$ e dessa forma concluímos que $\operatorname{ImT}$ é um subespaço de codimensão 1. Notemos que, para cada $\left(x_{n}\right)_{n} \in c$, vale

$$
\left\|T\left(\left(x_{n}\right)_{n}\right)\right\|_{\infty}=\sup \left(\left\{\left|-x_{1}\right|\right\} \cup\left\{\left|x_{n}\right|: n \in \mathbb{N}\right\}\right)=\sup \left(\left\{\left|x_{n}\right|: n \in \mathbb{N}\right\}\right)=\left\|\left(x_{n}\right)_{n}\right\|_{\infty}
$$

Com isso, mostramos que $T$ é uma isometria e concluímos que $T$ é um deslocamento isométrico 
em $C(X)$. Ao consideramos $X_{0}, \psi, w$ e $p$ como no exemplo 6, verificamos que $T$ é um deslocamento isométrico do tipo I. Por outro lado, seja $X_{0}=X$ e $\psi: X \longrightarrow X$ a função dada por

$$
\psi(x)=\left\{\begin{array}{rll}
x-1, & \text { se } & x \in \mathbb{N} \backslash\{1\} \\
1, & \text { se } x=1 \\
\infty, & \text { se } x=\infty
\end{array}\right.
$$

Vamos definir ainda $w: X \longrightarrow \mathbb{C}$, de modo que $w(1)=-1$ e $w(x)=1$ para todo $x \in X \backslash\{1\}$. Com isso, vemos através da definição 19 que T é um deslocamento isométrico do tipo II. Para conhecer melhor as possibilidades para um deslocamento isométrico, vamos a mais um exemplo.

Exemplo 8. Seja $X$ como no exemplo 6, vamos identificar $C(X)$ com c. Seja T o operador linear definido por

$$
\begin{array}{ccc}
T: C(X) & \longrightarrow & C(X) \\
x=\left(x_{n}\right)_{n} & \longmapsto & \left(-\frac{x_{1}+x_{2}}{2}, x_{1}, x_{2}, \ldots\right)
\end{array}
$$

A demonstração de que $T$ é um deslocamento isométrico não é muito diferente do exemplo 7 , exceto pela prova de que

$$
\bigcap_{n=1}^{\infty} \operatorname{Im} T^{n}=\{0\} .
$$

Mostremos esse fato. Suponhamos $\left(x_{n}\right)_{n} \in \bigcap_{n=1}^{\infty} I m T^{n}$. Usando o princípio da indução finita, é facil ver que

$$
x_{k}=-\frac{x_{k+1}+x_{k+2}}{2}, \text { para cada } k \in \mathbb{N} .
$$

Da igualdade acima concluimos diretamente que $\left|x_{k}\right| \leq \max \left\{\left|x_{k+1}\right|,\left|x_{k+2}\right|\right\}$, para todo $k \in \mathbb{N}$. Por (4.6), notemos ainda que, dado um $k \in \mathbb{N}$ arbitrário, entre os três termos consecutivos

$$
x_{k}, x_{k+1}, x_{k+2},
$$

pelo menos um deles tem parte real maior ou igual a zero e pelo menos um deles tem parte real menor ou igual a zero. Para a parte imaginária cabe a mesma conclusão. Como $\left(x_{n}\right)_{n}$ é uma sequência convergente, isso só é possível se

$$
\lim _{n \rightarrow \infty} x_{n}=0
$$

Seja $\varepsilon>0$ arbitrário. Segue que existe $N_{0} \in \mathbb{N}$ de modo que $\left|x_{n}\right|<\varepsilon$ para todo $n \in \mathbb{N}, n \geq N_{0}$. Por 4.6, fixado $k \in \mathbb{N}$ arbitrário, existe uma subsequência $\left(x_{n_{j}}\right)_{j \in \mathbb{N}}$ de $\left(x_{n}\right)_{n}$, de modo que

$$
\left|x_{k}\right| \leq\left|x_{n_{j}}\right| \text {, para todo } j \in \mathbb{N} \text {. }
$$

Assim $\left|x_{n}\right|<\varepsilon$, para todo $n \in \mathbb{N}$. Provamos que, para cada $\varepsilon>0,\left|x_{n}\right|<\varepsilon$ para todo $n \in \mathbb{N}$. Assim podemos concluir que $\left|x_{n}\right|=0$ para todo $n \in \mathbb{N}$ e dessa forma $\left(x_{n}\right)_{n}$ é a sequência nula. Logo

$$
\bigcap_{n=1}^{\infty} \operatorname{Im} T^{n}=\{0\} .
$$


Com isso, vamos admitir que $T$ é um deslocamento isométrico em $C(X)$. Ao definirmos $X_{0}, \psi$, $w$ e p como no exemplo 6, é fácil mostrar que $T$ é um deslocamento isométrico do tipo I. Vamos ver que $T$ não é um deslocamento isométrico do tipo II. Suponhamos, por absurdo, $\psi: X \longrightarrow X$ continua e sobrejetora e $w: X \longrightarrow \mathbb{C}$ contínua satisfazendo

$$
|w(x)|=1 \text { e } T f(x)=w(x) f(\psi(x)) \text { para todo } x \in X, f \in C(X) .
$$

Para $f=(0, i, 0, \ldots)$ e $x=1$, a igualdade acima só é possivel se $\psi(1)=2$ e $|w(1)|=\frac{1}{2}$. Uma contradição, já que estávamos supondo $|w(x)|=1$, para todo $x \in X$.

Os lemas 8 e 9 são resultados básicos decorrentes do teorema 21, o objetivo deles é descrever propriedades básicas da função $\psi$, a qual é induzida por um deslocamento isométrico definido em $C(X)$. Como podemos notar, o Lema de Urysohn é frequentemente usado nos resultados, principalmente na prova por absurdo.

Lema 8. Suponhamos que $T$ seja um deslocamento isométrico em $C(X)$ e $f \in C(X) \cap I m T$. Então $|f|$ é constante em cada fibra de de $\psi$ (olhar observação 8). Fixado $y \in X$, uma fibra de $\psi$ é um conjunto da forma $\psi^{-1}(y)=\left\{x \in X_{0}: \psi(x)=y\right\}$.

Demonstração. Suponhamos $f \in C(X) \cap \operatorname{Im} T$, digamos que $f=T g$. Daí $\psi: X_{0} \longrightarrow X$ verifica:

$$
f(x)=T g(x)=w(x) g(\psi(x)), \text { para todo } x \in X_{0} .
$$

Como $w: X_{0} \longrightarrow \mathbb{K}$ é tal que $|w(x)|=1$ para todo $x \in X_{0}$, segue que

$$
|f(x)|=|T g(x)|=|g(\psi(x))|, \text { para todo } x \in X_{0} .
$$

Sejam $x_{1}, x_{2}$ elementos de $X_{0}$ numa mesma fibra de $\psi$, ou seja, tais que $\psi\left(x_{1}\right)=\psi\left(x_{2}\right)$, usando a igualdade anterior temos

$$
\left|f\left(x_{1}\right)\right|=\mid g\left(\psi ( x _ { 1 } ) | = | g \left(\psi\left(x_{2}\right)|=| f\left(x_{2}\right) \mid .\right.\right.
$$

Assim $|f|$ é constante em cada fibra de $\psi$.

Lema 9. Seja $T$ um deslocamento isométrico em $C(X)$. Sejam $X_{0}, \psi$ e w como no teorema 21, vamos provar que:

(i) $\psi^{-1}(x)$ tem, no máximo, dois elementos, para cada $x \in X$;

(ii) Se existem $a, b \in X_{0}$, distintos tais que $\psi(a)=\psi(b)=x_{0}$, então $\psi^{-1}(x)$ é um conjunto unitário, para cada $x \in X \backslash\left\{x_{0}\right\}$;

(iii) Se $X_{0} \neq X$ (T é do tipo I), então $\psi$ é injetora, logo é um homeomorfismo.

Demonstração. (i) Vamos provar esse resultado por absurdo. Suponhamos que existam três pontos $x_{1}, x_{2}$ e $x_{3}$ em $X_{0}$, dois a dois distintos, satisfazendo $\psi\left(x_{1}\right)=\psi\left(x_{2}\right)=\psi\left(x_{3}\right)$. Pelo Lema de Urysohn, existem funções $f_{1}: X \longrightarrow[0,1], f_{2}: X \longrightarrow[0,1]$ em $C(X)$ tais que

$$
f_{1}(x)=1, \text { se } x \in\left\{x_{1}\right\} \text { e } f_{1}(x)=0, \text { se } x \in\left\{x_{2}, x_{3}\right\}
$$




$$
f_{2}(x)=1, \text { se } x \in\left\{x_{3}\right\} \text { e } f_{2}(x)=0, \text { se } x \in\left\{x_{1}, x_{2}\right\} .
$$

Como $T$ é um operador de codimensão 1 e $\left\{f_{1}, f_{2}\right\}$ é linearmente independente, existem $\lambda$ e $\beta$ constantes em $\mathbb{K}$, não ambas nulas de modo que $\lambda f_{1}+\beta f_{2} \in \operatorname{Im} T$. Pelo lema 8 , devemos ter

$$
\left|\lambda f_{1}\left(x_{1}\right)+\beta f_{2}\left(x_{1}\right)\right|=\left|\lambda f_{1}\left(x_{2}\right)+\beta f_{2}\left(x_{2}\right)\right|=\left|\lambda f_{1}\left(x_{3}\right)+\beta f_{2}\left(x_{3}\right)\right| .
$$

Das igualdades acima concluímos que $\lambda=\beta=0$, uma contradição.

(ii) Vamos provar este item por absurdo. Suponhamos $x_{1}, x_{2} \in X$ distintos de maneira que $\psi^{-1}\left(x_{1}\right)$ e $\psi^{-1}\left(x_{2}\right)$ ambos tenham dois elementos distintos. Digamos que existem $a, b, c$ e $d$ em $X_{0}$, dois a dois distintos, satisfazendo:

$$
\psi(a)=\psi(b)=x_{1} \text { e } \psi(c)=\psi(d)=x_{2} .
$$

Pelo Lema de Urysohn, existem funções $g_{1}: X \longrightarrow[0,1], g_{2}: X \longrightarrow[0,1]$ em $C(X)$ tais que

$$
\begin{aligned}
& g_{1}(x)=1, \text { se } x \in\{b\} \text { e } g_{1}(x)=0, \text { se } x \in\{a, c, d\} ; \\
& g_{2}(x)=1, \text { se } x \in\{a, c\} \text { e } g_{2}(x)=0, \text { se } x \in\{b, d\} .
\end{aligned}
$$

Novamente, por ser $T$ um operador de codimensão 1 e por ser $\left\{g_{1}, g_{2}\right\}$ um conjunto linearmente independente, vem que existem $\lambda, \beta$ constantes em $\mathbb{K}$, não ambas nulas, tais que $\left(\lambda g_{1}+\beta g_{2}\right) \in$ $\operatorname{ImT}$. Pelo lema 8, segue que

$$
\left|\lambda g_{1}(a)+\beta g_{2}(a)\right|=\left|\lambda g_{1}(b)+\beta g_{2}(b)\right| \text { e }\left|\lambda g_{1}(c)+\beta g_{2}(c)\right|=\left|\lambda g_{1}(d)+\beta g_{2}(d)\right| .
$$

Dessas igualdades concluímos que $\beta=\lambda=0$, uma contradição. Assim provamos (ii) por absurdo.

(iii) Suponhamos que $T$ seja do tipo I, daí como vimos no teorema 21, $X_{0}=X \backslash\{p\}$ e $\{p\}$ é um conjunto aberto e fechado. Assim, pelo Lema de Urysohn, existe $f: X \longrightarrow[0,1]$ em $C(X)$ tal que

$$
f(p)=1 \text { e } f(x)=0, \text { se } x \in X_{0} .
$$

Suponhamos, por absurdo, que $\psi$ não é injetora, ou seja, que existem $a, b \in X_{0}$ distintos satisfazendo $\psi(a)=\psi(b)$. Pelo Lema de Urysohn, existe $g: X \longrightarrow[0,1]$ em $C(X)$ tal que

$$
g(b)=1 \text { e } g(x)=0, \text { se } x \in\{p, a\}
$$

Como o conjunto $\{f, g\}$ é lineamente independente em $C(X)$ e $T$ é um operador de codimensão 1 , existem constantes $\lambda, \beta$ em $\mathbb{K}$, não ambas nulas, tais que $\lambda f+\beta g \in \operatorname{ImT}$. Daí, pelo lema 8 temos

$$
|\lambda f(a)+\beta g(a)|=|\lambda f(b)+\beta g(b)| .
$$

Essa última igualdade nos diz que $\beta=0$. Assim $\lambda f \in \operatorname{Im} T$ e $\lambda \neq 0$. Como $\operatorname{Im} T$ é um subespaço de $C(X)$, segue que $f \in \operatorname{ImT}$, isso é uma contradição com o corolário 10. Assim concluímos que $\psi: X_{0} \longrightarrow X$ é injetora. Como já tínhamos que $\psi$ é contínua e sobrejetora, $X_{0}$ é compacto 
e $X$ é Hausdorff, segue que $\psi$ é um homeomorfismo.

Observação 10. Se $T: C(X) \longrightarrow C(X)$ é do tipo I, então $X \backslash X_{0}=\{p\}$, onde $p$ é um ponto isolado de $X$. Pelo lema 9, $\psi: X_{0} \longrightarrow X$ é um homeomorfismo, dai existe um único ponto $p_{1}$ em $X_{0}$ de modo que $\psi\left(p_{1}\right)=p$. Pela continuidade e injetividade de $\psi$, concluímos que $p_{1}$ é um ponto isolado de $X_{0}$. Ainda mais, como $X_{0}$ é aberto em $X, p_{1}$ é um ponto isolado de $X$ satisfazendo $\psi\left(p_{1}\right)=p$.

Portanto, repetindo o raciocínio acima para $p_{1}$ no lugar de $p$, concluímos que existe $p_{2}$ ponto isolado de $X$ de maneira que $\psi\left(p_{2}\right)=p_{1}$. Como $p \notin X_{0}$, temos $p \neq p_{1}$ e $p \neq p_{2}$. Além disso, pela injetividade de $\psi$, temos $p_{1} \neq p_{2}$. Usando o mesmo raciocínio, construímos indutivamente uma sequência

$$
\left(p, p_{1}, p_{2}, \ldots, p_{n}, \ldots\right)
$$

de pontos isolados em $X$ - distintos dois a dois. Além disso, os pontos da sequência satisfazem $\psi\left(p_{n+1}\right)=p_{n}$, para cada $n \in \mathbb{N}$ e $\psi\left(p_{1}\right)=p$. Com isso, concluímos que se $C(X)$ admite um deslocamento isométrico do tipo $I$, então $X$ tem um conjunto infinito de pontos isolados.

Terminaremos a seção mostrando como a classificação dos deslocamentos em $C(X)$ pode ser uma ferramenta útil. Através dessa abordagem, no teorema 22, provaremos que $C([a, b])$ não admite deslocamentos isométricos. Para chegar nesse resultado, precisamos apenas provar um simples lema, o qual exibe uma propriedade da função $\psi$ nos deslocamentos isométricos do tipo II.

Lema 10. Seja $T$ um deslocamento isométrico tipo II em $C(X)$. Então $\psi: X \longrightarrow X$ não é injetora.

Demonstração. Suponhamos, por absurdo, que $\psi: X \longrightarrow X$ seja injetora. Como $X$ é um compacto Hausdorff, $\psi$ é homeomorfismo e satisfaz

$$
(T f)(x)=w(x) f(\psi(x)) \text {, para todo } x \in X \text { e } f \in C(X) .
$$

Seja $g \in C(X)$ uma função qualquer. Notemos que

$$
f=\frac{g \circ \psi^{-1}}{w \circ \psi^{-1}} \in C(X)
$$

e, evidentemente, $T f=g$. Pela arbitrariedade, concluímos que $T$ é sobrejetora. Uma contradição, pois $\operatorname{Im} T$ tem codimensão 1.

Teorema 22. Não existe um deslocamento isométrico definido no espaço $C([a, b])$, sendo $[a, b]$ um intervalo fechado da reta real.

Demonstração. Faremos a demonstração por absurdo. Suponhamos que $C([a, b])$ admite um deslocamento isométrico $T$. Como $[a, b]$ não tem pontos isolados, $T$ deve ser do tipo II. Pelo lema $10, \psi:[a, b] \longrightarrow[a, b]$ não é injetora. Consideremos $y, z \in[a, b]$ distintos tais que $\psi(y)=\psi(z)$. Podemos supor, sem perder generalidade, que $y<z$. Fixemos $x_{0} \in[a, b] \operatorname{com} y<x_{0}<z$. 
Pelo lema 9 (i) e (ii),

$$
\left.\psi\left(x_{0}\right) \neq \psi(z) \text { e } \psi \text { é injetora quando restrita ao intervalo }\right] y, z[\text {. }
$$

Suponhamos, sem perder generalidade, que $\psi\left(x_{0}\right)>\psi(y)=\psi(z)$. Daí, como $\psi$ é contínua, $\psi$ preserva conexidade e compacidade. Segue que o conjunto $\psi\left(\left[y, x_{0}\right]\right)$ é um intervalo fechado da reta real contido em $[a, b]$. Além disso, esse intervalo deve conter $\left[\psi(y), \psi\left(x_{0}\right)\right]$. Por analogia, o conjunto $\psi\left(\left[x_{0}, z\right]\right)$ é um intervalo fechado da reta real contido em $[a, b]$ e que contém o intervalo $\left[\psi(y), \psi\left(x_{0}\right)\right]$.

Como estamos supondo $\psi(y)<\psi\left(x_{0}\right)$ e $\psi(z)<\psi\left(x_{0}\right)$, existe $v \in[a, b]$ tal que

$$
\psi(z)=\psi(y)<v<\psi\left(x_{0}\right)
$$

Pelo fato de $\left[\psi(y), \psi\left(x_{0}\right)\right] \subseteq \psi\left(\left[y, x_{0}\right]\right)$ e $\left[\psi(y), \psi\left(x_{0}\right)\right] \subseteq \psi\left(\left[x_{0}, z\right]\right)$, existem $\left.u \in\right] y, x_{0}\left[, u^{\prime} \in\right] x_{0}, z[$ tais que

$$
\psi(u)=\psi\left(u^{\prime}\right)=v
$$

isso contradiz (4.7).

\subsection{Estudo dos deslocamentos isométricos tipo I}

A meta desta seção é trazer uma condição suficiente para garantir a existência de um deslocamento isométrico do tipo I em $C(X)$, isso será feito no teorema 24 . Ao fim, daremos um exemplo bem interessante, aplicando as ferramentas desenvolvidas nesta parte do trabalho. Apesar de fazer essa proposta, vamos iniciar com uma definição e um resultado que pressupõe que $C(X)$ já admite um deslocamento isométrico. Vale enfatizar que nesta seção, da mesma maneira como na anterior, $\mathbb{K}=\mathbb{R}$ ou $\mathbb{K}=\mathbb{C}$.

Definição 20. Seja $T$ um deslocamento isométrico do tipo I definido em $C(X)$, seja $X \backslash X_{0}=$ $\{p\}$ como no teorema 21. Definimos $\Gamma_{T}$, o funcional linear contínuo associado a $T$, da seguinte forma:

$$
\begin{aligned}
\Gamma_{T}: C(X) & \longrightarrow \mathbb{K} \\
f & \longmapsto(T f)(p)
\end{aligned}
$$

Os próximos resultados que trataremos nesta seção podem ser vistos em [9].

Teorema 23. Seja $T$ um deslocamento isométrico do tipo I definido em $C(X)$. Suponhamos $X \backslash X_{0}=\{p\}$, seguindo a notação do teorema 21, e $\Gamma_{T} \equiv 0$. Então o conjunto $D=$ $\left\{p, \psi^{-1}(p), \psi^{-2}(p), \ldots, \psi^{-n}(p), \ldots\right\}$ é denso em $X$. Portanto $X$ é homeomorfo a uma compactificação dos naturais.

Demonstração. Suponhamos $g \in C(X)$ tal que $g(x)=0$, para todo $x \in \bar{D}$, mostremos que $g$ é necessariamente a função nula. Consideremos $\chi_{p}: X \longrightarrow \mathbb{K}$ a função característica de $\{p\}$, isto é,

$$
\chi_{p}(p)=1 \text { e } \chi_{p}(x)=0 \text {, para todo } x \in X \backslash\{p\} .
$$


Afirmo que existem constantes $\lambda, \beta \in \mathbb{K}$, não ambas nulas, de maneira que $\lambda \chi_{p}+\beta g \in \operatorname{ImT}$. De fato, se $g \equiv 0$ essas constantes trivialmente existem. Por outro lado, se $g \not \equiv 0$, o conjunto $\left\{g, \chi_{p}\right\}$ é lineamente independente em $C(X)$, já que $g(p)=0$ e $\chi_{p}(p)=1$. Daí, a existência dessas constantes segue do fato de $\operatorname{Im} T$ ter codimensão 1.

Daí, fixemos $\lambda, \beta \in \mathbb{K}$, não ambas nulas, tais que $\lambda \chi_{p}+\beta g \in \operatorname{ImT}$. Pelo colorário 10, $\chi_{p}$ não está em $\operatorname{Im} T, \operatorname{logo} \beta \neq 0$. Tomando $\alpha=\frac{\lambda}{\beta}$ e tendo em mente que $\operatorname{Im} T$ é subespaço de $C(X)$, segue que

$$
\alpha \chi_{p}+g \in \operatorname{Im} T \text {. }
$$

Suponhamos que $\alpha \chi_{p}+g=T h$, para $h \in C(X)$. Temos, por hipótese,

$$
0=\Gamma_{T}(h)=T h(p)=\alpha \chi_{p}(p)+g(p)=\alpha .
$$

Assim $g \in \operatorname{Im} T, g=T h$. Sabemos que

$$
g(x)=T h(x)=w(x) h(\psi(x)), \text { para todo } x \in X_{0} .
$$

Veja que, por ser $\psi$ um homeomorfismo, segue que

$$
h=\frac{g \circ \psi^{-1}}{w \circ \psi^{-1}} .
$$

Notemos que $\psi^{-1}(D) \subseteq D$, pela definição do conjunto $D$. Como $\psi^{-1}$ é um função contínua, segue que $\psi^{-1}(\bar{D}) \subseteq \bar{D}$. Como $g$ é uma função que se anula em $\bar{D}$, devemos ter (pela igualdade acima) que $h$ se anula em $\bar{D}$.

Tomamos $g$ uma função qualquer que se anula em $\bar{D}$ e concluímos que $g \in \operatorname{ImT}$. Depois disso, provamos que se $g=T h$, então $h$ se anula em $\bar{D}$. Com isso, $h \in \operatorname{Im} T$ e $g \in \operatorname{Im} T^{2}$. Com esse argumento, provamos por indução que

$$
g \in \bigcap_{n=1}^{\infty} \operatorname{I} m T^{n} .
$$

Como $T$ é um deslocamento isométrico, devemos ter $g \equiv 0$. Portanto toda função que se anula em $\bar{D}$ deve ser nula em $X$. Como $\bar{D}$ é um conjunto fechado, segue pelo Lema de Urysohn que $\bar{D}=X$.

Da observação 10, cada elemento do conjunto $D$ é um ponto isolado em $X$. Daí $D$, como subespaço topológico de $X$, é o espaço discreto - e evidentemente é homeomorfo aos naturais. Como vimos, $D$ é denso em $X$ e $X$ é um espaço topológico compacto e Hausdorff. Logo $X$ é homeomorfo a uma compactificação dos naturais.

O próximo teorema é, em certo sentido, uma recíproca do último resultado. Esse teorema se adequa a proposta da seção.

Teorema 24. Seja $X$ um espaço topológico compacto e Hausdorff com um ponto isolado $p$, seja ainda $X_{0}=X \backslash\{p\}$. Suponhamos que existe um homeomorfismo $\psi: X_{0} \longrightarrow X$ de modo que o conjunto $D=\left\{p, \psi^{-1}(p), \psi^{-2}(p), \ldots, \psi^{-n}(p), \ldots\right\}$ seja denso em $X$. Nessas condições, existe um deslocamento isométrico $T$ definido em $C(X)$ tal que $\Gamma_{T} \equiv 0$. 
Demonstração. Vamos definir $T$ da seguinte forma

$$
\begin{aligned}
T: C(X) & \longrightarrow C(X) \\
f & \longmapsto g
\end{aligned}
$$

de modo que $g(p)=0$ e $g(x)=f(\psi(x))$, para todo $x \in X_{0}$. Como $p$ é ponto isolado em $X$, a função $g$ é, de fato, contínua e $T$ está bem definida. Além disso, através da definição verificamos, sem dificuldades, que $T$ é um operador linear. Como $\psi$ é sobrejetora é verdade que

$$
\begin{aligned}
\sup \left\{|T f(x)|: x \in X_{0}\right\} & =\sup \left\{|f \circ \psi(x)|: x \in X_{0}\right\}= \\
& =\sup \{|f(x)|: x \in X\}= \\
& =\|f\|_{\infty} .
\end{aligned}
$$

Daí, como $|T f(p)|=0$, concluímos que $T$ é uma isometria.

Por definição, se $g \in \operatorname{Im} T$, então $g(p)=0$. Ainda, pelo princípio de indução finita é simples mostrar que, se $g \in I m T^{n+1}$, então $g\left(\psi^{-n}(p)\right)=0$. Assim consideremos

$$
g \in \bigcap_{n=1}^{\infty} \operatorname{I} m T^{n} .
$$

Pelo que observamos acima, segue que $g$ se anula em $D$. Assim $g$ é uma função contínua que coincide com a função nula em um conjunto denso em $X$, logo $g \equiv 0$.

Resta-nos mostrar que $\operatorname{Im} T$ é um subespaço de $C(X)$ com codimensão 1. Para tal, vamos mostrar que

$$
\operatorname{Im} T=\{h \in C(X): h(p)=0\} .
$$

A inclusão $\operatorname{Im} T \subseteq\{h \in C(X): h(p)=0\}$ segue diretamente da definição de $T$. Por outro lado, seja $h \in C(X) \operatorname{com} h(p)=0$. Consideremos a função

$$
g=h \circ \psi^{-1} \in C(X)
$$

Notemos que, se $x \in X_{0}, T g(x)=h \circ \psi^{-1}(\psi(x))=h(x)$. Temos ainda, $\operatorname{Tg}(p)=0=h(p)$. Segue que $h=T g$, daí $h \in \operatorname{Im} T$ e assim provamos a igualdade (4.8). A demonstração de que o subespaço $\{h \in C(X): h(p)=0\}$ tem codimensão 1 é simples e será omitida.

O próximo lema foge do contexto dos deslocamentos isométricos, trata-se de um simples resultado de análise na reta real. Esse dará suporte para o próximo corolário desta seção.

Lema 11. O conjunto $\{n \sqrt{2}+m: n, m \in \mathbb{Z}$ e $n \geq 0\}$ é denso na reta real.

Demonstração. De fato, consideremos $y \in \mathbb{R}$ e $\varepsilon>0$ arbitrários. Seja $k \in \mathbb{Z}$ de forma que $k \leq y<k+1$. Sabemos que

$$
\lim _{n \rightarrow \infty}(\sqrt{2}-1)^{n}=0 .
$$


Seja $n_{0} \in \mathbb{N}$, ímpar e que verifica $(\sqrt{2}-1)^{n_{0}}<\varepsilon$. Claro que

$$
(\sqrt{2}-1)^{n_{0}}=\sum_{j=0}^{n_{0}} C_{n_{0}, j}(-1)^{j}(\sqrt{2})^{n_{0}-j}, \text { sendo } C_{n_{0}, j}=\frac{n_{0} !}{j !\left(n_{0}-j\right) !} .
$$

Através da expressão acima e do fato de $n_{0}$ ser ímpar, fica claro que existem $s, t \in \mathbb{Z}, s>0$ tais que $(\sqrt{2}-1)^{n_{0}}=s \sqrt{2}+t$. Daí observe que um dos termos da sequência

$$
k, k+(s \sqrt{2}+t), k+2(s \sqrt{2}+t), k+3(s \sqrt{2}+t), \ldots, k+n(s \sqrt{2}+t), \ldots
$$

pertence ao intervalo $] y-\varepsilon ; y+\varepsilon[$ e ao conjunto $\{n \sqrt{2}+m: n, m \in \mathbb{Z}$ e $n \geq 0\}$.

Consideremos $X$ um espaço topológico compacto, Hausdorff e com uma infinidade de componentes conexas. Na década de 80 , muitos matemáticos que estudavam os deslocamentos em espaços de Banach conjecturaram que $C(X)$ não admitiria um deslocamento isométrico nessas condições. Um importante matemático, entre esses, é J. R. Holub (artigo [13]). O próximo corolário é uma forma de ver que essa conjectura é falsa.

Corolário 11. Existe um espaço topológico X, compacto, Hausdorff e com um número infinito de componentes conexas de maneira que $C(X)$ admite um deslocamento isométrico.

Demonstração. Consideremos $\mathbb{N}$ o espaço topológico dos números naturais munido da topologia discreta, na qual todo subconjunto dos números naturais é aberto. Seja $\beta \mathbb{N}$ o compactificado de Stone-Cech de $\mathbb{N}$, logo $\mathbb{N}$ é denso em $\beta \mathbb{N}$. Vejamos que para cada $n_{0} \in \mathbb{N},\left\{n_{0}\right\}$ é aberto em $\beta \mathbb{N}$. Para isso, consideremos a função

$$
\alpha: \mathbb{N} \longrightarrow[0,1]
$$

definida por:

$$
\alpha(n)=\left\{\begin{array}{lll}
1, & \text { se } & n \in \mathbb{N} \backslash\left\{n_{0}\right\} \\
0, & \text { se } & n=n_{0}
\end{array}\right.
$$

Como $\mathbb{N}$ é o espaço discreto, a função $\alpha$ é trivialmente contínua. Seja $\hat{\alpha}$ a extensão contínua de $\alpha$ a $\beta \mathbb{N}$. Então o conjunto $\hat{\alpha}^{-1}\left(\left[0, \frac{1}{2}[)\right.\right.$ é aberto em $\beta \mathbb{N}$. Como $\hat{\alpha}$ estende $\alpha$, o único natural pertencente ao conjunto $\hat{\alpha}^{-1}\left(\left[0, \frac{1}{2}[)\right.\right.$ é $n_{0}$. Daí, pelo fato de $\mathbb{N}$ ser denso em $\beta \mathbb{N}$, devemos ter $\hat{\alpha}^{-1}\left(\left[0, \frac{1}{2}[)=\left\{n_{0}\right\}\right.\right.$. Segue que para cada $n_{0} \in \mathbb{N},\left\{n_{0}\right\}$ é aberto em $\beta \mathbb{N}$ e que $\mathbb{N}$ é aberto em $\beta \mathbb{N}$.

Seja $S^{1}$ o círculo unitário, ou ainda,

$$
S^{1}=\left\{e^{2 \pi i x}: x \in[0,1]\right\}=\{z \in \mathbb{C}:|z|=1\} .
$$

Consideremos a função $f$ dada por:

$$
\begin{aligned}
f: \mathbb{N} & \longrightarrow S^{1} \\
n & \longmapsto e^{2 \pi i n \sqrt{2}}
\end{aligned}
$$

Evidentemente, a função $f$ é contínua e, pelo lema 11, Imf é um conjunto denso em $S^{1}$. Por ser $S^{1}$ um compacto Hausdorff, $f$ admite uma extensão contínua a $\beta \mathbb{N}$. Seja $\hat{f}: \beta \mathbb{N} \longrightarrow S^{1}$ a extensão contínua de $f$. Como $\beta \mathbb{N}$ é compacto e $\hat{f}$ é contínua, $\hat{f}(\beta \mathbb{N})$ é compacto em $S^{1}, \operatorname{logo}$ 
é fechado. Segue daí que

$$
\operatorname{Im} \hat{f}=\hat{f}(\beta \mathbb{N})=\overline{\hat{f}(\beta \mathbb{N})} \supseteq \overline{f(\mathbb{N})}=S^{1}
$$

e assim concluímos que $\hat{f}$ é sobrejetora. Vamos definir uma relação de equivalência $\sim$ em $\beta \mathbb{N}$. Sejam $x, y \in \beta \mathbb{N}$ arbitrários, então:

$$
x \sim y \Longleftrightarrow \begin{array}{rlll}
x, y \in \mathbb{N} & \text { e } & x=y \\
& \text { ou } & \\
x, y \in \beta \mathbb{N} \backslash \mathbb{N} & \text { e } & \hat{f}(x)=\hat{f}(y)
\end{array}
$$

É muito simples verificar que $\sim$ é, de fato, uma relação de equivalência. Consideremos o conjunto quociente $X=\beta \mathbb{N} / \sim$. Seja $q: \beta \mathbb{N} \longrightarrow X$ a aplicação quociente dada por

$$
\begin{aligned}
q: \beta \mathbb{N} & \longrightarrow X \\
x & \longmapsto[x] .
\end{aligned}
$$

Vamos munir $X$ da topologia quociente $\tau$, a topologia mais fina que torna $q$ contínua, dada por

$$
\tau=\left\{U \in \mathcal{P}(X): q^{-1}(U) \text { é aberto em } \beta \mathbb{N}\right\}
$$

Mostremos que o espaço topológico $X$ é Hausdorff. Temos que verificar 3 possíveis casos:

Primeiro Caso: Suponhamos $x, y \in \beta \mathbb{N} \backslash \mathbb{N}$ de maneira que $[x] \neq[y]$. Pela definição de $\sim$, devemos ter $\hat{f}(x) \neq \hat{f}(y)$. Daí, como $S^{1}$ é Hausdorff, existem abertos disjuntos $U$ e $V$ em $S^{1}$ tais que $\hat{f}(x) \in U$ e $\hat{f}(y) \in V$. Dessa forma, $\hat{f}^{-1}(U)$ e $\hat{f}^{-1}(V)$ são abertos disjuntos em $\beta \mathbb{N}$ tais que $x \in \hat{f}^{-1}(U)$ e $y \in \hat{f}^{-1}(V)$. Pela definição de $\tau$, os conjuntos $q\left(\hat{f}^{-1}(U)\right)$ e $q\left(\hat{f}^{-1}(V)\right)$ são abertos em $X$, disjuntos, tais que $[x] \in q\left(\hat{f}^{-1}(U)\right)$ e $[y] \in q\left(\hat{f}^{-1}(V)\right)$. E assim este caso está resolvido.

Segundo Caso: Suponhamos $x \in \beta \mathbb{N} \backslash \mathbb{N}$ e $y \in \mathbb{N}$, daí $[x] \neq[y]$. Nesse caso, notemos que $q^{-1}(\{[y]\})=\{y\}$ é aberto em $\beta \mathbb{N}$. Além disso, $q^{-1}(X \backslash\{[y]\})=\beta \mathbb{N} \backslash\{y\}$ é aberto em $\beta \mathbb{N}$. Daí $\{[y]\}$ e $X \backslash\{[y]\}$ são abertos disjuntos em $X$ e separam os pontos $[x]$ e $[y]$.

Terceiro Caso: Suponhamos $x \in \mathbb{N}, y \in \mathbb{N}$ distintos. Daí $[x] \neq[y]$. Neste caso, usando o que foi feito no segundo caso, sabemos que $\{[x]\}$ e $\{[y]\}$ são abertos em $X$, disjuntos e que separam os pontos dados.

Provamos assim que $X$ é Hausdorff. No meio dessa demonstração, vimos também que $\mathbb{N}$ é aberto em $X$ e, evidentemente, $\mathbb{N}$ é denso em $X$. Notemos que, do fato de $q$ ser contínua e $\beta \mathbb{N}$ compacto, concluímos que $X=q(\beta \mathbb{N})$ deve ser compacto. Além disso, $X \backslash \mathbb{N}$ é fechado em $X$, logo também é compacto. Seja $g$ a função dada por:

$$
\begin{aligned}
g: X & \longrightarrow S^{1} \\
& {[x] } \\
& \longmapsto \hat{f}(x)
\end{aligned}
$$


Notemos que, pela definição da relação $\sim, g$ está bem definida. A função $g$ é trivialmente contínua em $\mathbb{N}$, pois para cada $n \in \mathbb{N},\{[n]\}$ é aberto em $X$. Consideremos então $x \in \beta \mathbb{N} \backslash \mathbb{N}$ e suponhamos que $V$ seja um aberto em $S^{1}$ contendo o ponto $g([x])=\hat{f}(x)$. Como $\hat{f}$ é contínua, $\hat{f}^{-1}(V)$ é um aberto em $\beta \mathbb{N}$ que contém o ponto $x$. Notemos que $q\left(\hat{f}^{-1}(V)\right)$ é um aberto em $X$ contendo o ponto $[x]$ e que $g\left(q\left(\hat{f}^{-1}(V)\right)\right) \subseteq V$. Pela generalidade desse argumento, concluímos que a função $g$ é contínua em todo $X$. Por ser $\hat{f}$ sobrejetora, concluímos que $g$ é sobrejetora.

Sabemos que para cada $n \in \mathbb{N}$, vale

$$
g([n+1])=\hat{f}(n+1)=e^{2 \pi i \sqrt{2}(n+1)}=\hat{f}(n) e^{2 \pi i \sqrt{2}}=g([n]) e^{2 \pi i \sqrt{2}} .
$$

Seja $\left(n_{k}\right)_{k}$ uma sequência de números naturais arbitrária. Decorre da expressão acima que

$$
\left(g\left(\left[n_{k}\right]\right)\right)_{k} \text { converge se, e somente se, }\left(g\left(\left[n_{k}+1\right]\right)\right)_{k} \text { converge. }
$$

Notemos que a restrição $g_{\mid X \backslash \mathbb{N}}$ tem imagem densa em $S^{1}$, já que $\mathbb{N}$ é um conjunto enumerável e todo aberto não vazio de $S^{1}$ é um conjunto não enumerável. Além disso, $X \backslash \mathbb{N}$ é compacto, assim concluímos que $g_{\left.\right|_{X \backslash \mathbb{N}}}$ é sobrejetora. Tendo em mente a definição de $g$ e a compacidade dos conjuntos $X \backslash \mathbb{N}$ e $S^{1}$, concluímos que :

$$
g_{\left.\right|_{X \backslash \mathbb{N}}}: X \backslash \mathbb{N} \longrightarrow S^{1} \text { é um homeomorfismo. }
$$

Mostremos agora que, dada uma sequência qualquer de naturais $\left(\left[n_{k}\right]\right)_{k}$ em $X$,

$$
\left(\left[n_{k}\right]\right)_{k} \text { é convergente em } X \text { se, e somente se, }\left(\left[n_{k}+1\right]\right)_{k} \text { é convergente em } X \text {. }
$$

Suponhamos que $\left(\left[n_{k}\right]\right)_{k}$ seja uma sequência de números naturais convergente em $X$, de modo que $\lim _{k \rightarrow \infty}\left[n_{k}\right]=\left[x_{0}\right]$. Vamos supor que $\left[x_{0}\right] \in X \backslash \mathbb{N}$ e depois provar o caso $\left[x_{0}\right] \in \mathbb{N}$. Como $g$ é contínua, segue que $\left(g\left(\left[n_{k}\right]\right)\right)_{k}$ converge para $g\left(\left[x_{0}\right]\right)$ em $S^{1}$. Por $(4.9),\left(g\left(\left[n_{k}+1\right]\right)\right)_{k}$ converge em $S^{1}$, a saber para $y=e^{2 \pi i \sqrt{2}} g\left(\left[x_{0}\right]\right)$. Por (4.10), existe $\left[y_{0}\right] \in X \backslash \mathbb{N}$ de maneira que $g\left(\left[y_{0}\right]\right)=y$. Afirmo que $\lim _{k \rightarrow \infty}\left[n_{k}+1\right]=\left[y_{0}\right]$. De fato, suponhamos por absurdo que isso não seja verdade. Daí existe um aberto $V$ em $X$ de maneira que $\left[y_{0}\right] \in V$ e $\left\{k \in \mathbb{N}:\left[n_{k}+1\right] \in X \backslash V\right\}$ é infinito. Notemos que o conjunto $X \backslash V$ é compacto e, pela continuidade da função $g, g(X \backslash V)$ também o é. O conjunto $\left\{k \in \mathbb{N}: g\left(\left[n_{k}+1\right]\right) \in g(X \backslash V)\right\}$ é infinito e $\lim _{k \rightarrow \infty} g\left(\left[n_{k}+1\right]\right)=y$, $\operatorname{logo} y \in g(X \backslash V)$. Segue que existe $\left[n_{0}\right] \in(X \backslash V) \cap \mathbb{N}$ de maneira que $g\left(\left[n_{0}\right]\right)=y$. Notemos que o conjunto $V \cup\left\{\left[n_{0}\right]\right\}$ é um aberto contendo [yo] de modo que $\left\{k \in \mathbb{N}:\left[n_{k}+1\right] \in X \backslash\left(V \cup\left\{\left[n_{0}\right]\right\}\right)\right\}$ é infinito, pois a sequência $\left(\left[n_{k}+1\right]\right)_{k}$ certamente não tem uma infinidade de termos iguais a $\left[n_{0}\right]$. Novamente o compacto $g\left(X \backslash\left(V \cup\left\{\left[n_{0}\right]\right\}\right)\right)$ é tal que $\left\{k \in \mathbb{N}: g\left(\left[n_{k}+1\right]\right) \in g\left(X \backslash\left(V \cup\left\{\left[n_{0}\right]\right\}\right)\right)\right\}$ é infinito e $y \notin g\left(X \backslash\left(V \cup\left\{\left[n_{0}\right]\right\}\right)\right)$, uma contradição. Nessa prova consideramos $\left[x_{0}\right] \in X \backslash \mathbb{N}$, notemos que se $\lim _{k \rightarrow \infty}\left[n_{k}\right]=\left[x_{0}\right]$ e $\left[x_{0}\right] \in \mathbb{N}$, a sequência $\left(\left[n_{k}\right]\right)_{k}$ deve ser quase constante, bem como a sequência $\left(\left[n_{k}+1\right]\right)_{k}$, logo esse caso é resolvido de maneira trivial. A recíproca:

$$
\text { se }\left(\left[n_{k}+1\right]\right)_{k} \text { converge em } X \text {, então }\left(\left[n_{k}\right]\right)_{k} \text { converge em } X \text {. }
$$

é análoga e com isso demonstramos (4.11). Na demonstração de (4.11) provamos também, 
que dada uma sequência de naturais $\left(\left[n_{k}\right]\right)_{k}$, em $X$, de maneira que $\left(\left[n_{k}\right]\right)_{k}$ não tenha uma subsequência constante e dado um ponto $[x] \in X \backslash \mathbb{N}$, temos:

se $\left(g\left(\left[n_{k}\right]\right)\right)_{k}$ converge para $g([x])$, então $\left(\left[n_{k}\right]\right)_{k}$ converge para $[x]$.

Dessa maneira, vamos definir uma nova função $s: X \longrightarrow X$. Para isso, seja $[x] \in X$ arbitrário e suponhamos que $\left(\left[n_{k}\right]\right)_{k}$ seja uma sequência de naturais convergindo para $[x]$. Defino $s([x])$ como o ponto limite da sequência $\left(\left[n_{k}+1\right]\right)_{k}$, que sabemos ser convergente por (4.11). Para $[x] \in \mathbb{N}$, está claro que $s$ está bem definida. Suponhamos $[x] \in X \backslash \mathbb{N}$ arbitrário e $\left(\left[n_{k}\right]\right)_{k},\left(\left[m_{k}\right]\right)_{k}$ duas sequências de naturais em $X$, ambas convergindo para $[x]$. Digamos que $\lim _{k \rightarrow \infty}\left[n_{k}+1\right]=$

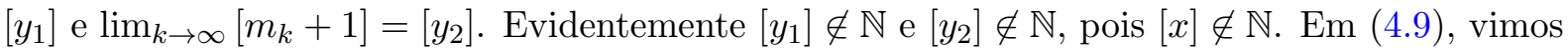
que

$$
g\left(\left[y_{1}\right]\right)=e^{2 \pi i \sqrt{2}} g([x])=g\left(\left[y_{2}\right]\right),
$$

logo, por (4.10), $\left[y_{1}\right]=\left[y_{2}\right]$. Com isso, a função $s$ está bem definida e sabemos que vale:

$$
g(s([x]))=e^{2 \pi i \sqrt{2}} g([x]), \text { para todo }[x] \in X .
$$

Vamos mostrar que $s$ é contínua. Se $[x] \in \mathbb{N}$, obviamente $s$ é contínua em $[x]$, pois $[x]$ é aberto em $X$. Resta mostrarmos que $s$ é contínua em $[x] \in X \backslash \mathbb{N}$ arbitrário. Suponhamos $[x] \in X \backslash \mathbb{N}$ e $V$ um aberto em $X$ de maneira que $s([x])=[y] \in V$, claramente $[y] \in X \backslash \mathbb{N}$. Por (4.10), $g(V \backslash \mathbb{N})$ é aberto em $S^{1}$. O conjunto $A_{0}=\left\{z \in S^{1}: e^{2 \pi i \sqrt{2}} z \in g(V \backslash \mathbb{N})\right\}$ também é aberto em $S^{1}$. Seja $A$ um aberto de $S^{1}$, que contém o ponto $e^{-2 \pi i \sqrt{2}} g([y])$ e que satisfaz $\bar{A} \subseteq A_{0}$, consideremos ainda o conjunto $U=g^{-1}(A)$, que é aberto em $X$. Evidentemente $[x] \in U$ e notemos que, se $[u] \in U \cap(X \backslash \mathbb{N})$, então $g(s([u]))=e^{2 \pi i \sqrt{2}} g([u]) \in g(V \backslash \mathbb{N})$, daí $s([u]) \in V$. Para concluir que $s$ é contínua em $[x]$, consideremos o conjunto

$$
I=\{[n] \in \mathbb{N} \cap U:[n+1] \notin V\} .
$$

Vamos provar, por absurdo, que esse conjunto é finito. De fato, suponhamos que $I$ seja infinito. Consideremos $\left(\left[n_{k}\right]\right)_{k}$, uma sequência de naturais em $I$ de modo que $\left[n_{j}\right] \neq\left[n_{t}\right]$ para todos $j, t \in \mathbb{N} \operatorname{com} j \neq t$. Notemos que o conjunto $\bar{A}$ é compacto, daí a sequência $\left(g\left(\left[n_{k}\right]\right)_{k}\right.$ admite uma subsequência convergente em $\bar{A}$. Sem perdermos generalidade, suponhamos que $\left(g\left(\left[n_{k}\right]\right)\right)_{k}$ converge para $w \in \bar{A}$. Seja $\left[x_{1}\right] \in X \backslash \mathbb{N}$ tal que $g\left(\left[x_{1}\right]\right)=w$, logo $w \in \bar{A} \subseteq A_{0}$. Portanto $g\left(s\left(\left[x_{1}\right]\right)\right)=e^{2 \pi i \sqrt{2}} g\left(\left[x_{1}\right]\right) \in g(V \backslash \mathbb{N})$ e assim concluímos que $s\left(\left[x_{1}\right]\right) \in V$. Por outro lado, decorre de $(4.12)$ que $\left(\left[n_{k}\right]\right)_{k}$ converge para $\left[x_{1}\right]$. Daí pela definição de $s$, segue que $\left(\left[n_{k}+1\right]\right)_{k}$ converge para $s\left(\left[x_{1}\right]\right)$. Notemos que $\left\{\left[n_{k}+1\right]: k \in \mathbb{N}\right\} \subseteq X \backslash V$, pois a sequência $\left(\left[n_{k}\right]\right)_{k}$ foi tomada em $I$. Vejamos que, por $X \backslash V$ ser fechado, $s\left(\left[x_{1}\right]\right) \in X \backslash V$, uma contradição. Para concluir que $s$ é contínua em $[x]$, basta notarmos que o conjunto $U \backslash I$ é um aberto contendo $[x]$ e $s(U) \subseteq V$.

Suponhamos que $[x] \in X \backslash \mathbb{N},[y] \in X \backslash \mathbb{N}$ e $s([x])=s([y])$. Daí, segue que

$$
g([x])=e^{-2 \pi i \sqrt{2}} g(s([x]))=e^{-2 \pi i \sqrt{2}} g(s([y]))=g([y]),
$$


$\operatorname{logo}[x]=[y]$. É claro que se $[x] \in \mathbb{N}$ ou $[y] \in \mathbb{N}, s([x])=s([y])$ implica que $[x]=[y]$. Assim, $s$ é injetora.

Notemos que, dado $[x] \in X \backslash \mathbb{N}$, existe uma sequência de naturais $\left(\left[n_{k}\right]\right)_{k}$ convergindo para $[x]$ de maneira que $\left[n_{k}\right] \neq[1]$, para todo $k \in \mathbb{N}$. Daí, decorre de $(4.11)$ que $\left(\left[n_{k}-1\right]\right)_{k}$ converge em $X$. Digamos que $\left(\left[n_{k}-1\right]\right)_{k}$ convirja para $[u]$, é imediato verificar que $s([u])=[x]$. Com isso, mostramos que $X \backslash \mathbb{N} \subseteq s(X)$. Daí, é fácil ver que $s(X)=X \backslash\{[1]\}$.

Tendo em mente que $X$ e $X \backslash\{[1]\}$ são ambos compactos Hausdorff, concluímos que

$$
s: X \longrightarrow X \backslash\{[1]\}
$$

é um homeomorfismo. Fazendo $D=\mathbb{N}, p=[1]$ e $\psi=s^{-1}$, segue do teorema 24 que $C(X)$ admite um deslocamento isométrico do tipo I. Isso demonstra este corolário, visto que $X$ possui um número infinito de componentes conexas.

\subsection{Estudo dos deslocamentos isométricos tipo II}

Agora, voltemos nossa atenção para os deslocamentos isométricos do tipo II. Vamos notar as consequências de $C(X)$ admitir esse tipo de deslocamento. Pelos lemas 9 e 10, sabemos que existe um único par de pontos distintos $a, b \in X$ de maneira que $\psi(a)=\psi(b)$. Consideremos $c=\psi(a)$. Com essa notação vamos provar os próximos resultados, os quais podem ser encontrados em [9].

Teorema 25. Seja $T$ um deslocamento isométrico do tipo II definido em $C(X)$, sejam a, $b, c \in X$ como acima e sejam $\psi$ e $w$ como no teorema 21. Vamos definir $F \subseteq X$ da seguinte forma

$$
F=\bigcup_{k=-\infty}^{\infty} \psi^{k}(c)
$$

Então $F$ é denso em $X$.

Demonstração. Pela definição de $F$, concluímos que $\psi(F) \subseteq F$. Daí, por ser $\psi$ contínua, segue que $\psi(\bar{F}) \subseteq \bar{F}$. Por outro lado, suponhamos que $\psi(x) \in \bar{F}$, vamos provar que $x \in \bar{F}$. Notemos que se $\psi(x)=c$, isso é imediato. Então suponhamos $\psi(x) \in \bar{F} \backslash\{c\} \subseteq \overline{F \backslash\{c\}}$, logo existe uma rede $\left(z_{\alpha}\right)_{\alpha \in D}$ em $F \backslash\{c\}$, convergindo para $\psi(x)$. Para cada $\alpha \in D, \psi^{-1}\left(z_{\alpha}\right)$ é um conjunto unitário. Observemos que, pela definição de $F,\left(\psi^{-1}\left(z_{\alpha}\right)\right)_{\alpha \in D}$ é uma rede em $F$. Como $\bar{F}$ é compacto em $X,\left(\psi^{-1}\left(z_{\alpha}\right)\right)_{\alpha \in D}$ admite subrede convergente para $x_{0} \in \bar{F}$. Sem perda de generalidade e usando uma notação mais simples, suponhamos que $\left(\psi^{-1}\left(z_{\alpha}\right)\right)_{\alpha \in D}$ converge para $x_{0} \in \bar{F}$. Pela continuidade de $\psi$, segue que a rede

$$
\left(\psi\left(\psi^{-1}\left(z_{\alpha}\right)\right)\right)_{\alpha \in D}=\left(z_{\alpha}\right)_{\alpha \in D}
$$

converge para $\psi\left(x_{0}\right)$. Como $X$ é Hausdorff, devemos ter $\psi\left(x_{0}\right)=\psi(x)$, mas $\psi(x) \neq c$, portanto $x=x_{0} \in \bar{F}$. Mostramos que $\psi(\bar{F}) \subseteq \bar{F}$ e que se $\psi(x) \in \bar{F}$, então $x \in \bar{F}$. Tendo em mente que $\psi$ é sobrejetora, concluímos que $\psi(\bar{F})=\bar{F}$ e $\psi(X \backslash \bar{F})=X \backslash \bar{F}$. 
Vejamos agora que $\bar{F}=X$. Suponhamos, por absurdo, $\bar{F} \neq X$, daí existe $g \in C(X)$ não nula tal que $g(x)=0$, para todo $x \in \bar{F}$. Seja $h: X \longrightarrow \mathbb{K}$ a função definida por

$$
h(x)=\left\{\begin{array}{rll}
0, & \text { se } & x \in \bar{F} \\
\frac{g\left(\psi^{-1}(x)\right)}{w\left(\psi^{-1}(x)\right)}, & \text { se } & x \in X \backslash \bar{F}
\end{array}\right.
$$

Vejamos que $h$ é contínua em cada $x_{0} \in \bar{F}$. Se isso não fosse verdade, teríamos que existe uma rede $\left(x_{\alpha}\right)_{\alpha \in D}$ em $X$ convergindo para $x_{0}$, enquanto $\left(h\left(x_{\alpha}\right)\right)_{\alpha \in D}$ não converge para $h\left(x_{0}\right)=0$. Sem perder generalidade, podemos supor que existe $\varepsilon>0$ tal que $\left|h\left(x_{\alpha}\right)\right|>\varepsilon$ para cada $\alpha \in D$, pois isso vale para alguma subrede de $\left(h\left(x_{\alpha}\right)\right)_{\alpha \in D}$. Logo, pela definição de $h, x_{\alpha} \notin \bar{F}$, para cada $\alpha \in D$. Segue que $\psi^{-1}\left(x_{\alpha}\right)$ é um conjunto unitário para cada $\alpha \in D$. Como $X$ é compacto, $\left(\psi^{-1}\left(x_{\alpha}\right)\right)_{\alpha \in D}$ admite uma subrede convergente em $X$. Novamente, sem perda de generalidade, suponhamos que $\left(\psi^{-1}\left(x_{\alpha}\right)\right)_{\alpha \in D}$ converge para $z \in X$. Como $\psi$ é contínua, concluímos que $\left(\psi\left(\psi^{-1}\left(x_{\alpha}\right)\right)\right)_{\alpha \in D}=\left(x_{\alpha}\right)_{\alpha \in D}$ converge para $\psi(z)$, daí por ser $X$ Hausdorff, segue que $\psi(z)=x_{0}$. Provamos na primeira parte que $\psi(X \backslash \bar{F})=X \backslash \bar{F}$, logo $z \in \bar{F}$. Veja que, por ser $g$ contínua, concluímos que

$$
\left|h\left(x_{\alpha}\right)\right|=\left|g\left(\psi^{-1}\left(x_{\alpha}\right)\right)\right| \rightarrow|g(z)|=0, \text { pois } z \in \bar{F} .
$$

Isso contradiz a hipótese, daí devemos concluir que $h$ é contínua em cada $x_{0} \in \bar{F}$.

Agora suponhamos, por absurdo, que existe $x_{0} \in X \backslash \bar{F}$ de maneira que $h$ não é contínua em $x_{0}$. Nessas condições, sabemos que existe uma rede $\left(x_{\alpha}\right)_{\alpha \in D}$ convergindo para $x_{0}$, enquanto $\left(h\left(x_{\alpha}\right)\right)_{\alpha \in D}$ não converge para $h\left(x_{0}\right)$. Como $X \backslash \bar{F}$ é aberto em $X$, podemos supor, sem perda de generalidade, que $x_{\alpha} \in X \backslash \bar{F}$ para cada $\alpha \in D$. Sem perda de generalidade, podemos supor também que existe $\varepsilon>0$ de modo que $\left|h\left(x_{\alpha}\right)-h\left(x_{0}\right)\right|>\varepsilon$, para todo $\alpha \in D$, pois esses resultados valem para alguma subrede de $\left(x_{\alpha}\right)_{\alpha \in D}$. Segue que $\psi^{-1}\left(x_{\alpha}\right)$ é um conjunto unitário para cada $\alpha \in D$. Análogo ao que foi feito quando $x_{0} \in \bar{F}$, concluímos que $\left(\psi^{-1}\left(x_{\alpha}\right)\right)_{\alpha \in D}$ admite uma subrede convergente em $X$, vamos supor que $\left(\psi^{-1}\left(x_{\alpha}\right)\right)_{\alpha \in D}$ converge para $z \in X$. Pela continuidade de $\psi$, segue que $\left(\psi\left(\psi^{-1}\left(x_{\alpha}\right)\right)\right)_{\alpha \in D}=\left(x_{\alpha}\right)_{\alpha \in D}$ converge para $\psi(z)$ e assim, devemos ter $\psi(z)=x_{0}$. Como $\psi(\bar{F})=\bar{F}$, certamente $z \in X \backslash \bar{F}$. Pela continuidade de $g$ e de $w$, temos:

$$
h\left(x_{\alpha}\right)=\frac{g\left(\psi^{-1}\left(x_{\alpha}\right)\right)}{w\left(\psi^{-1}\left(x_{\alpha}\right)\right)} \rightarrow \frac{g(z)}{w(z)}=h\left(x_{0}\right) .
$$

Isso contradiz o fato de $\left(h\left(x_{\alpha}\right)\right)_{\alpha \in D}$ não convergir para $h\left(x_{0}\right)$. Assim $h$ é contínua em cada $x_{0} \in X \backslash \bar{F}$. Portanto $h \in C(X)$. Pelo fato de

$$
T f(x)=w(x) f(\psi(x)), \text { para todos } x \in X, f \in C(X),
$$

fica claro que $T h=g$. Mostramos que para $g \in C(X)$, que se anula em $\bar{F}$, existe $h \in C(X)$ tal que $T h=g$. Notemos que $h \in C(X)$ e $h$ se anula em $\bar{F}$, daí pelo mesmo raciocínio existe $\hat{h} \in C(X)$ que se anula em $\bar{F}$ de maneira que $T \hat{h}=h$, donde concluímos que $h \in \operatorname{ImT}$ e $g \in I m T^{2}$. Fazendo este raciocínio indutivamente, concluímos que

$$
g \in \bigcap_{n=1}^{\infty} I m T^{n} .
$$


Segue que $g$ é a função nula, uma contradição. A contradição veio do fato de supor $\bar{F} \neq X$.

Do teorema 25 decorrem, diretamente, os dois corolários a seguir.

Corolário 12. Se $X$ é um espaço topológico compacto, Hausdorff e $C(X)$ admite um deslocamento isométrico do tipo II, então X é separável.

Corolário 13. Se $X$ é um espaço topológico compacto, Hausdorff, sem pontos isolados e $C(X)$ admite um deslocamento isométrico, então X é separável.

Vimos que se $X$ é um espaço topológico compacto, Hausdorff e $C(X)$ admite um deslocamento isométrico do tipo I, então $X$ é homeomorfo a $X \backslash\{p\}$, sendo $p$ um ponto isolado em $X$ e $\psi$ o homeomorfismo. O próximo teorema mostra que se $C(X)$ admite um deslocamento isométrico do tipo II, então $X$ é homeomorfo a um espaço quociente obtido por uma relação de equivalência que identifica dois pontos de $X$.

Teorema 26. Seja $X$ um espaço topológico compacto, Hausdorff e suponhamos que $C(X)$ admite um deslocamento isométrico tipo II. Então existem pontos distintos $a, b \in X$ tais que $X$ é homeomorfo ao espaço quociente $Q=X / \sim$, uma vez que a relação $\sim$ é dada por

$$
x \sim y \text { se, e somente se, } x=y \text { ou }\{x, y\}=\{a, b\}, \text { para cada } x, y \in X .
$$

Demonstração. Pelos lemas 9 e 10, existem pontos distintos $a, b \in X$ tais que $\psi(a)=\psi(b)$. Além disso, para cada $x, y \in X, \psi(x) \neq \psi(y)$ desde que $\{x, y\} \neq\{a, b\}$. Obviamente a relação $\sim$, descrita no enunciado, define uma relação de equivalência para esses pontos $a$ e $b$. Daí podemos falar em $Q=X / \sim$, o conjunto quociente. A aplicação quociente é dada por

$$
\begin{aligned}
q: X & \longrightarrow Q \\
x & \longmapsto[x] .
\end{aligned}
$$

Consideremos o espaço topológico quociente $Q$, munido com a topologia

$$
\tau_{q}=\left\{U \in \mathcal{P}(Q): q^{-1}(U) \text { é aberto em } \mathrm{X}\right\}
$$

Através da definição de $\sim$ e do fato de $X$ ser compacto e Hausdorff, provamos facilmente que $Q$ é compacto e Hausdorff. Vamos definir ainda, a função

$$
\begin{aligned}
\tilde{\psi}: Q & \longrightarrow X \\
{[x] } & \longmapsto \psi(x) .
\end{aligned}
$$

Pela definição de $\sim$, concluímos que $\tilde{\psi}$ está bem definida e é injetora. Como $\psi$ é sobrejetora, segue que $\tilde{\psi}$ é sobrejetora. Já sabemos que $Q$ e $X$ são compactos e Hausdorff, logo para mostrar que $\tilde{\psi}$ é homeomorfismo basta provar que $\tilde{\psi}$ é contínua.

Consideremos $[x] \in Q,[x] \neq[a]$ arbitrário e seja $\theta$ um aberto em $X$ contendo $\tilde{\psi}([x])=\psi(x)$. Como $\psi: X \longrightarrow X$ é contínua, existe $V_{1}$ aberto em $X$, contendo $x$ de maneira que $\psi\left(V_{1}\right) \subseteq \theta$. Como $x \neq a, x \neq b$ e $X$ é Hausdorff, existe $V_{2}$ aberto em $X$, contendo $x$ e satisfazendo $a \notin V_{2}$ e $b \notin V_{2}$. Daí, o conjunto $V=q\left(V_{1} \cap V_{2}\right)$ é um aberto em $Q$, contendo o ponto $[x]$ e que satisfaz $\tilde{\psi}(V) \subseteq \theta$. Segue que $\tilde{\psi}$ é contínua em todo $[x] \in Q$, diferente de $[a]$. 
Seja $\theta$ um aberto em $X$, contendo o ponto $\psi(a)=\psi(b)$. Pela continuidade de $\psi$ no ponto $a$, existe um aberto $U_{1}$ em $X$, contendo o ponto $a$, de modo que $\psi\left(U_{1}\right) \subseteq \theta$. Pela continuidade de $\psi$ no ponto $b$, existe um aberto $U_{2}$ em $X$, contendo o ponto $b$ e que satisfaz $\psi\left(U_{2}\right) \subseteq \theta$. Notemos que $U=q\left(U_{1} \cup U_{2}\right)$ é um aberto em $Q$, contendo o ponto $[a]$ e que satisfaz $\tilde{\psi}(U) \subseteq \theta$. Segue que $\tilde{\psi}$ é contínua em $Q$ e, pelo que foi comentado, é um homeomorfimo.

O próximo resultado segue do teorema 26. Temos ainda que comparar a homologia de grupos dos espaços com seus respectivos espaços quociente e concluir que $X$ não é homeomorfo a nenhum de seus espaços quociente - exclusivamente com a relação de equivalência que identifica dois pontos distintos. Os conceitos de grupo fundamental e homologia de grupos são da área de topologia algébrica e se distanciam do foco deste trabalho. Para conhecer mais, esses conceitos podem ser vistos em [14], nos dois primeiros capítulos.

Corolário 14. O espaço $C(X)$ não admite deslocamento isométrico, quando $X$ é

1. a n-esfera $S^{n}$;

2. $o$ n-cubo $[a, b]^{n}$ 


\section{Referências Bibliográficas}

[1] R. E. Megginson. An Introduction to Banach Space Theory. Springer, 1998.

[2] R. Engelking. General Topology. Heldermann Verlag Berlin - Sigma Series in Pure Mathematics. Vol 6, 1989.

[3] S. P. Moshokoa, M. Rajagopalan e K. Sundaresan (2011): Shifts on Products of Banach spaces, Quaestiones Mathematicae, 34:3, 327-333.

[4] L. Pontryagin, Topological Groups, Second Edition, Gordon and Breach Science Publishers, New York, 1966.

[5] Pei-Kee Lin (2011): Isometric Shifts on $(c \oplus X)_{\infty}$. Journal of Mathematical Analysis and Applications, 384, 198-203.

[6] M. Rajagopalan, K. Sundaresan (2009): Generalised shifts on Cartesian products, Indian J. Pure Appl. Math 40; 183-190.

[7] Themistocles, M. Rassias e K. Sundaresan (2001) : Generalized backward shifts on Banach Spaces, J. Math Anal. Appl., 260; 36-45.

[8] W. Holsztynski (1966): Continuous mappings induced by isometries of spaces of continuous functions, Studia Math 26; 133-136.

[9] A. Gutek, D. Hart, J. Jamison, M. Rajagopalan (1991): Shift operators on Banach Spaces, J. Funct. Anal., 101; 97-119.

[10] J. Lindenstrauss, L. Tzafriri. Classical Banach Spaces - sequence spaces. Springer-Verlag. Vol 1, New York, 1977.

[11] John L. Kelley. General Topology. Springer-Verlag, 1955.

[12] J. A. Clarkson, Uniformly convex spaces, Trans. of the Amer. Math Soc. 40(1936), p. 396414.

[13] J.R. Holub (1988), On shift operators, Canad. Math. Bull. 31; 85-94.

[14] A. Hatcher, Algebraic topology. Cambridge University Press, 2002. 